\title{
Minimizing the makespan in a flexible flowshop with sequence dependent setup times, uniform machines, and limited buffers
}

\author{
Bret Crowder \\ West Virginia University
}

Follow this and additional works at: https://researchrepository.wvu.edu/etd

\section{Recommended Citation}

Crowder, Bret, "Minimizing the makespan in a flexible flowshop with sequence dependent setup times, uniform machines, and limited buffers" (2006). Graduate Theses, Dissertations, and Problem Reports. 4220.

https://researchrepository.wvu.edu/etd/4220

This Thesis is protected by copyright and/or related rights. It has been brought to you by the The Research Repository @ WVU with permission from the rights-holder(s). You are free to use this Thesis in any way that is permitted by the copyright and related rights legislation that applies to your use. For other uses you must obtain permission from the rights-holder(s) directly, unless additional rights are indicated by a Creative Commons license in the record and/ or on the work itself. This Thesis has been accepted for inclusion in WVU Graduate Theses, Dissertations, and Problem Reports collection by an authorized administrator of The Research Repository @ WVU. For more information, please contact researchrepository@mail.wvu.edu. 


\title{
Minimizing the Makespan in a Flexible Flowshop with Sequence Dependent Setup Times, Uniform Machines, and Limited Buffers
}

\author{
Bret Crowder
}

\author{
Thesis submitted to the \\ College of Engineering and Mineral Resources \\ West Virginia University \\ In partial fulfillment of the requirements
}

For the degree of

\begin{abstract}
Master of Science
In

Industrial Engineering

Wafik Iskander, Ph.D., Chair

Bhaskaran Gopalakrishnan, Ph.D.

Alan R. McKendall, Ph.D.
\end{abstract}

Department of Industrial and Management Systems Engineering

Morgantown, West Virginia

2006

Keywords: Flexible Flowshop, Makespan, Sequence Dependent Setup Times, Uniform Machines, Scheduling, Simulated Annealing, Lower Bound, Limited Buffers 


\section{Abstract}

\section{Minimizing the Makespan in a Flexible Flowshop with Sequence Dependent Setup Times, Uniform Machines, and Limited Buffers}

\section{Bret Crowder}

This research addresses the problem of minimizing the makespan in a flexible flowshop with sequence dependent setup times, uniform machines, and limited buffers. A mathematical model was developed to solve this problem. The problem is NP-Hard in the strong sense and only very small problems could be solved optimally. For exact methods, the computation times are long and not practical even when the problems are relatively small. Two construction heuristics were developed that could find solutions quickly. Also a simulated annealing heuristic was constructed that improved the solutions obtained from the construction heuristics. The combined heuristics could compute a good solution in a short amount of time. The heuristics were tested in three different environments: 3 stages, 4 stages, and 5 stages. To assess the quality of the solutions, a lower bound and two simple heuristics were generated for comparison purposes. The proposed heuristics showed steady improvement over the simple heuristics. When compared to the lower bounds, the heuristics performed well for the smaller environment, but the performance quality decreased as the number of stages increased. The combination of these heuristics defiantly shows promise for solving the problem. 


\section{Acknowledgments}

I would like to thank Dr. Iskander for guidance and support while working on this thesis. His door was always open and he was always willing to help. While in graduate school I've enjoyed taking his classes and working with him in the Industrial Assessment Center. I would like to thank Dr. Gopala for his input on my research and for the responsibilities that he has given me through the Industrial Assessment Center. While working under him I've learned a great deal about energy and about the softer skills of dealing with people that are not taught in the classroom. Even after leaving WVU I will continue to benefit from my experience in the IAC working under these two Professors.

I would like to thank Dr. McKendall for his input on my research and for his guidance while taking his classes in the graduate program. His IENG 350 course sparked my interest in the operations research field and was one of the factors in my decision to come to graduate school.

Last I would like to thank Dr. Plummer for taking me in at the IAC. Working at the IAC helped me finance my degree. While working under Dr. Plummer I learned about focusing on the important issues and how to work with people. Also working with the other students in the IAC helped me to integrate into the graduate program. 
Table of Contents:

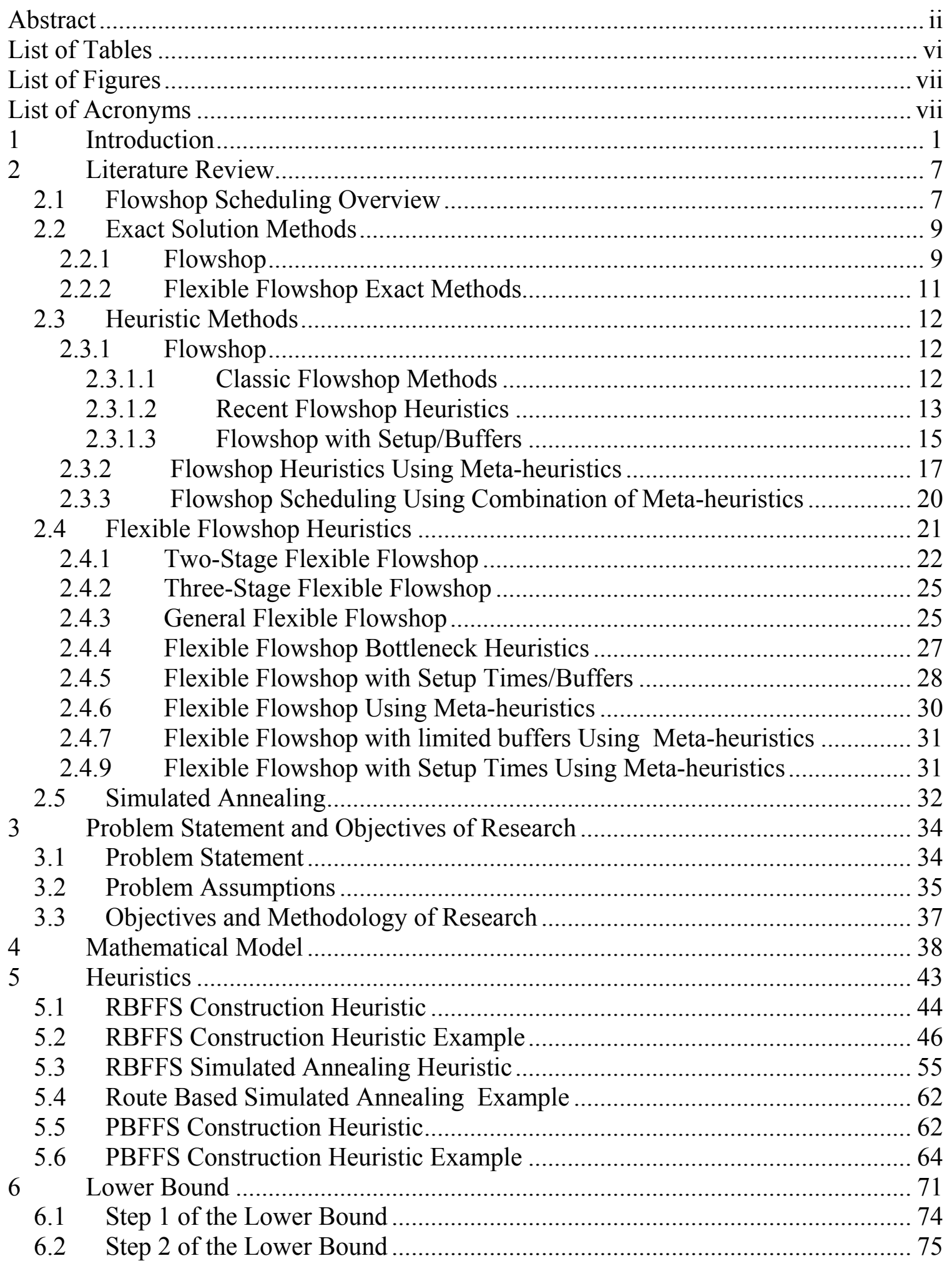




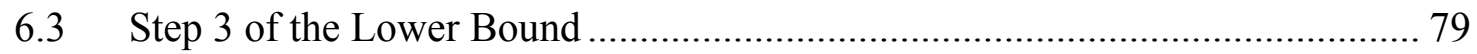

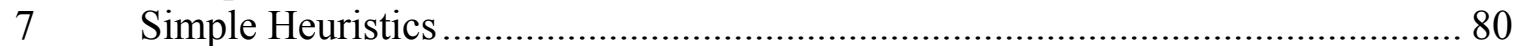

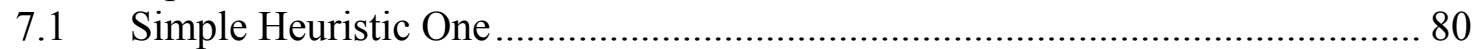

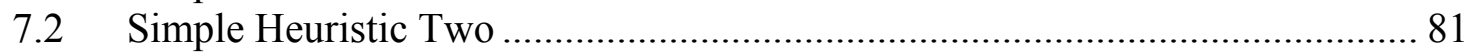

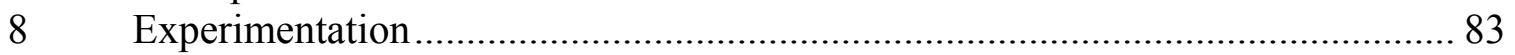

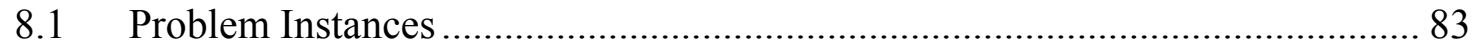

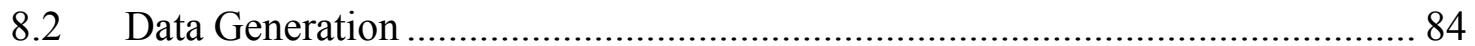

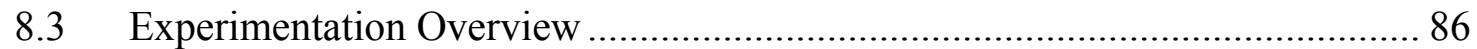

8.4 Simulated Annealing Parameters .................................................................. 86

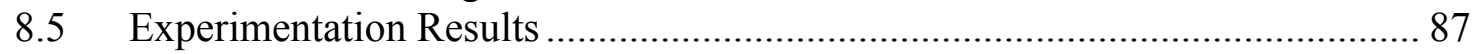

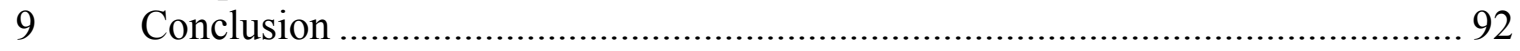

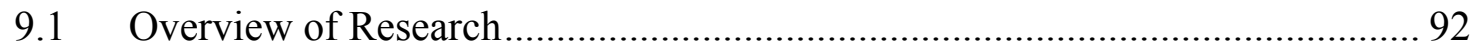

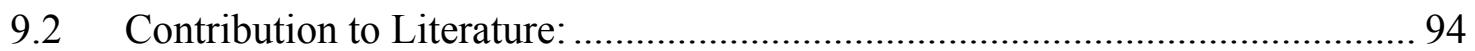

9.3 Recommendations for Future Research:.................................................... 94

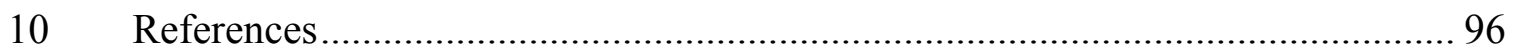

Appendix A: Experimental Results ..................................................................... 107 


\section{List of Tables}

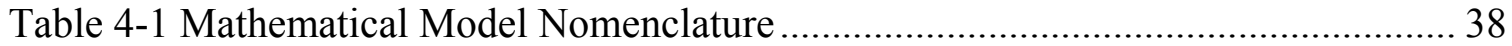

Table 5-1 Construction Heuristic Ex. Process Times Stage 1 ....................................... 46

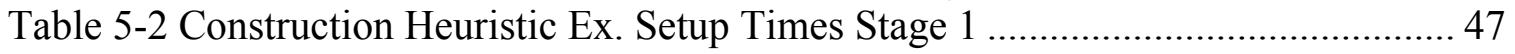

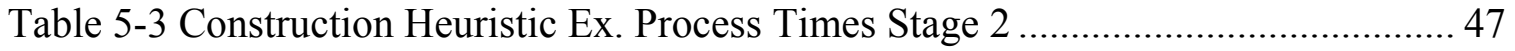

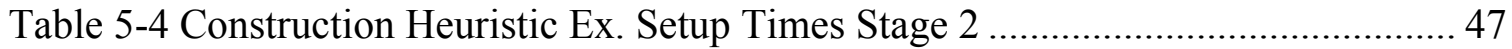

Table 5-5 Construction Heuristic Ex. Process Times Stage 3 ..................................... 47

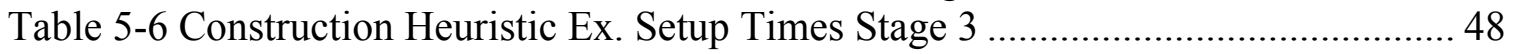

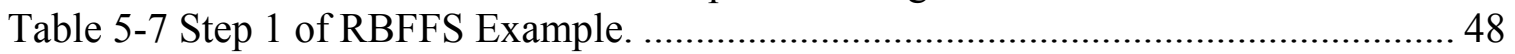

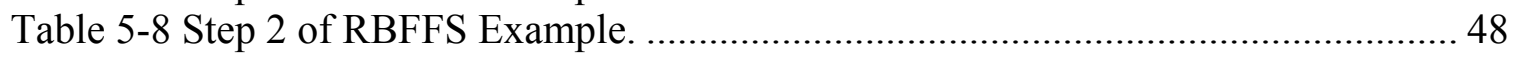

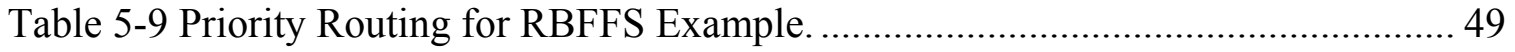

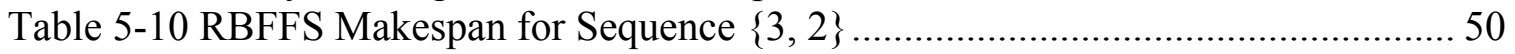

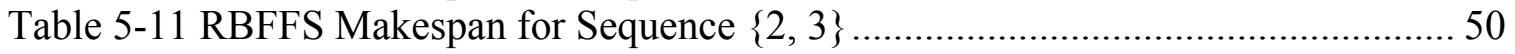

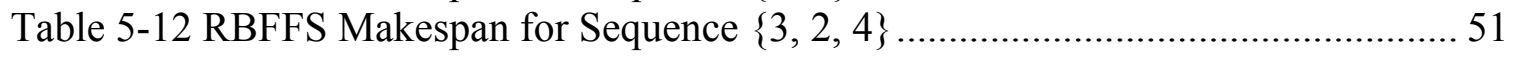

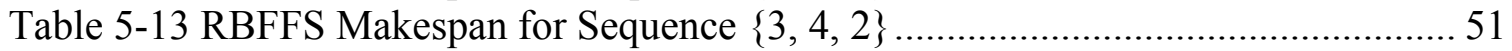

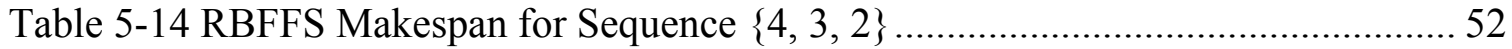

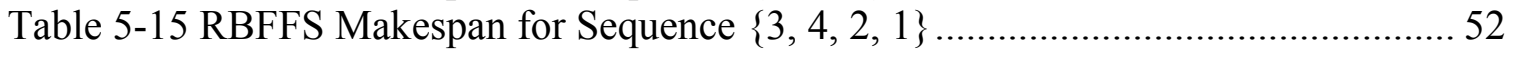

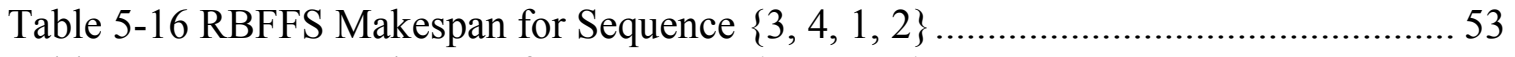

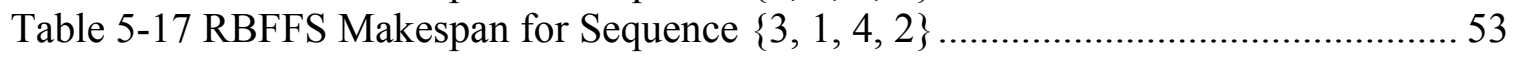

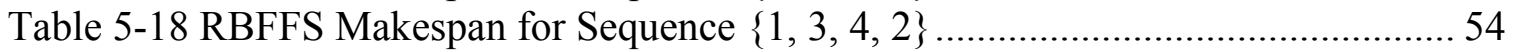

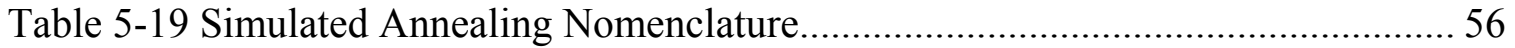

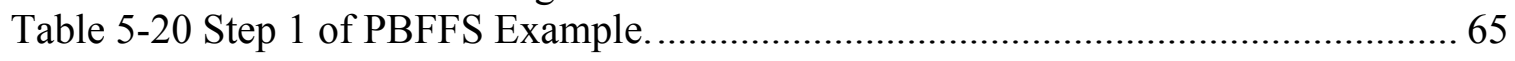

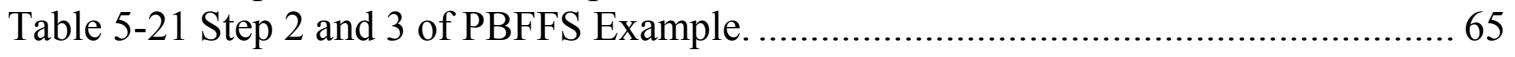

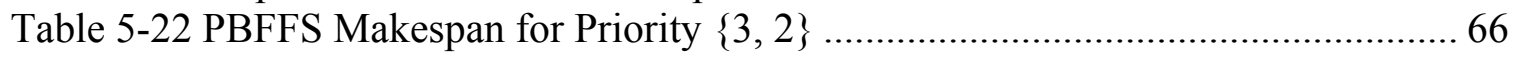

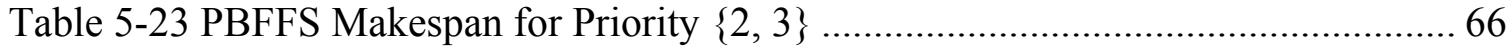

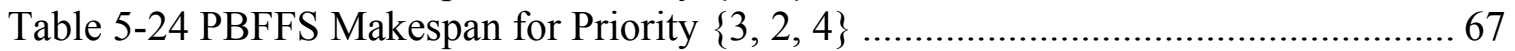

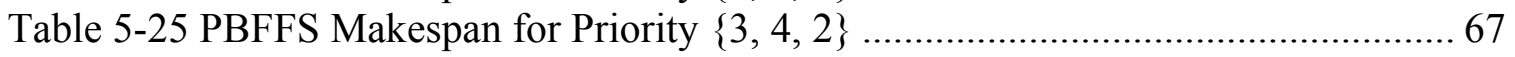

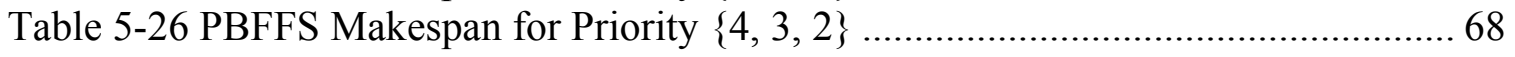

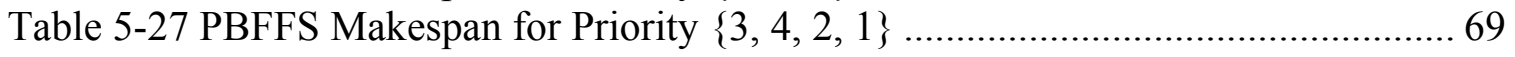

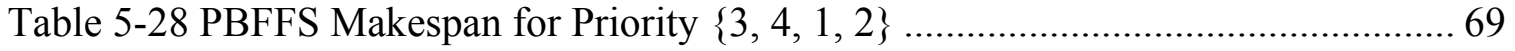

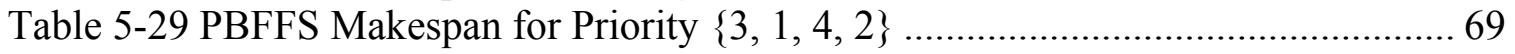

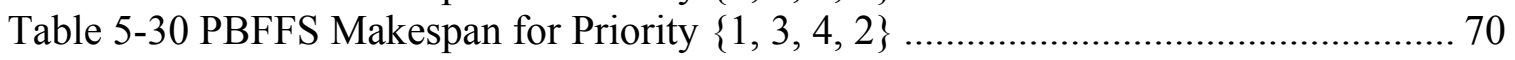

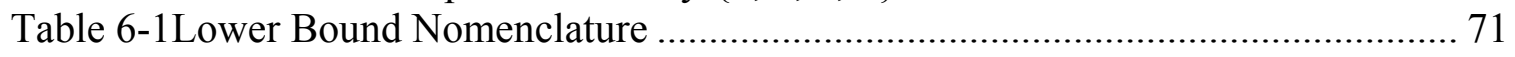

Table 6-2 Lower Bound Example Stage 1 Processing Times........................................ 73

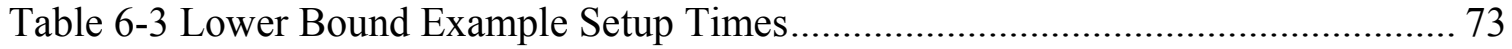

Table 6-4Lower Bound Example Stage 2 Processing Times......................................... 73

Table 6-5 Lower Bound Example Stage 3 Processing Times......................................... 73

Table 6-6 Lower Bound Example Stage 4 Processing Times........................................... 73

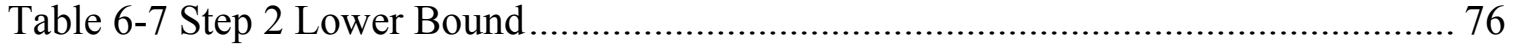

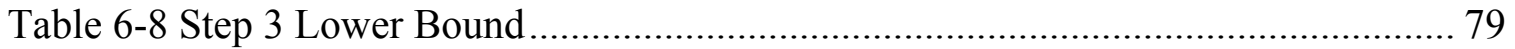

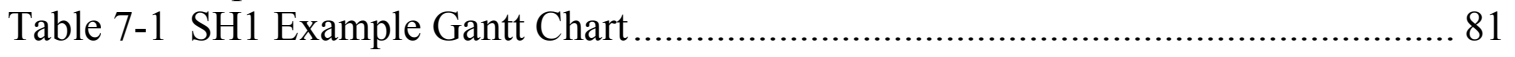

Table 7-2 Sum of Base Processing Times ................................................................ 81

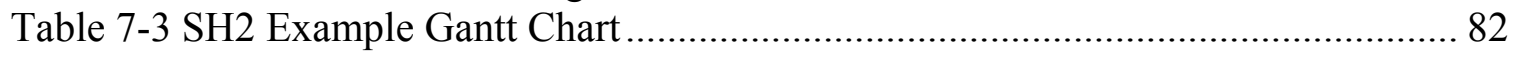

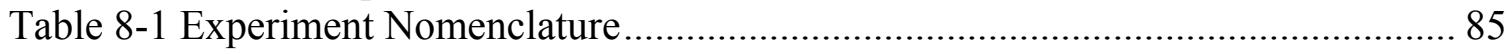

Table 8-2 Experimentation Results for the 3-1-2 Environment................................... 89 
Table 8-3 Experimentation Results for the 2-1-2-1 Environment

\section{List of Figures}

Figure 1.1 Complexity Hierarchy of Deterministic Scheduling Environment (Pinedo, 2002) 3

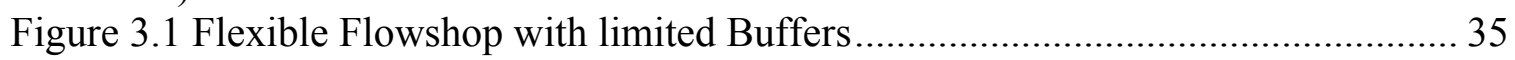

Figure 5.1 Simulated Annealing Process Flow Diagram4 ......................................... 59

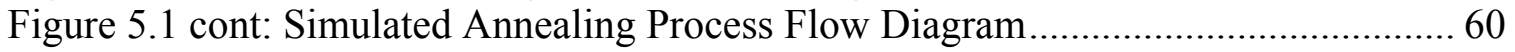

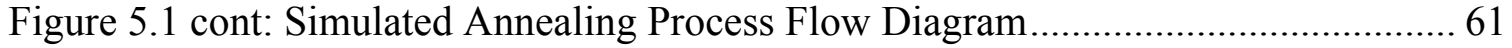

\section{List of Acronyms}

\begin{tabular}{|c|c|c|}
\hline AIS & $=$ & Artificial Immune System \\
\hline $\mathrm{BB}$ & $=$ & Branch and Bound \\
\hline CDS & $=$ & Flowshop algorithm developed by Campbell, Dudek, and Smith \\
\hline CDS2 & $=$ & Combination of CDS with RA \\
\hline CMD & $=$ & Cumulative Minimum Deviation \\
\hline ECTH & $=$ & Earliest Completion Time Heuristic \\
\hline EFM & $=$ & Earliest Finished Machine \\
\hline FAMH & $=$ & Fastest Available Machine Heuristic \\
\hline FCFS & $=$ & First Come First Serve \\
\hline FFS & $=$ & Flexible Flowshop \\
\hline FIFO & $=$ & First In First Out \\
\hline FS & $=$ & Flowshop \\
\hline GA & $=$ & Genetic Algorithm \\
\hline \multirow[t]{2}{*}{ GCMD } & $=$ & Generalized Cumulative Minimum Deviation Rule developed by \\
\hline & & Narasimham \\
\hline Ho & $=$ & Algorithm developed by Ho (1995) \\
\hline LB & $=$ & Lower Bound \\
\hline LBJD & $=$ & Least Total Weighted Between-Jobs Delay \\
\hline LP & $=$ & Linear Programming \\
\hline LPT & $=$ & Longest Processing Time \\
\hline LR & $=$ & Lagrangian Relaxation \\
\hline
\end{tabular}




\begin{tabular}{|c|c|c|}
\hline LSM & $=$ & Latest Start Machine \\
\hline MD & $=$ & Minimum Deviation \\
\hline MH & $=$ & Mixed Heuristic \\
\hline MIP & $=$ & Mixed Integer Program \\
\hline NEH & $=$ & Flowshop algorithm developed by Nawaz, Enscore, and Ham \\
\hline NP & $=$ & Non-polynomial \\
\hline PAM & $=$ & Modified version of Palmer's algorithm \\
\hline PBFFS & $=$ & Priority Based Construction Heuristic \\
\hline PBFFS_SA & $=$ & Priority Based Simulated Annealing Heuristic \\
\hline PBI & $=$ & Progressive Bottleneck Improvement \\
\hline RA & $=$ & Rapid Access algorithm developed by Dannenbring \\
\hline \multirow[t]{2}{*}{ RAES } & $=$ & Rapid Access with Extensive Search algorithm developed by \\
\hline & & Dannenbring \\
\hline RBFFS & $=$ & Route Based Construction Heuristic \\
\hline RBFFS_SA & $=$ & Route Based Simulated Annealing Heuristic \\
\hline SA & $=$ & Simulated Annealing \\
\hline SDST & $=$ & Sequence Dependent Setup Times \\
\hline SH1 & $=$ & First Come First Serve Simple Heuristic \\
\hline $\mathrm{SH} 2$ & $=$ & Largest Processing Time Simple Heuristic \\
\hline SPT & $=$ & Shortest Processing Time \\
\hline TS & $=$ & Tabu Search \\
\hline TSP & $=$ & Traveling Salesman Problem \\
\hline
\end{tabular}




\section{Introduction}

Scheduling and sequencing is a form of decision making that plays an important role in manufacturing and service industries. Scheduling is the allocation of resources to perform a set of tasks over time and sequencing is the order in which the tasks are performed. Resources can be a number of things such as, machines, workstations in a factory, operators, personal, delivery trucks, office workers, taxis, or runways in an airport. The products that are being processed are usually referred to as jobs and each one of these jobs has a specific set of tasks assigned to it. These problems arise whenever there is a choice to be made on the order in which a number of tasks can be performed and by using which resources. Many scheduling problems are solved by chance, scheduling the tasks in the order they arrive (First in First Out) (Conway et al., 1967). This works in some instances such as purchasing tickets to an event or placing an order at a fast food restaurant. However, in a manufacturing environment this approach can waste valuable time and money. Even when scheduling systems are used, many times they are developed by the operator with objectives that differ from the company's objectives. The operators may only be worried about their workstation and choose jobs based on what will be easiest on them, without concern about other stations. In a flowshop environment this may result in starving stations downstream, or blocking stations upstream. Sometimes these scheduling rules will result in local optima, but when they are all put together the entire system will suffer. In today's global market place with fierce competition, an efficient schedule can be very useful in reducing processing costs and lead times. Scheduling also has an important role in lean manufacturing, which uses scheduling to level production loads. As long as companies and businesses face capacity 
constraints, there will be a need for effective scheduling. Scheduling and sequencing involve decision making and by studying these problems we can learn about decision making, and apply it to other areas as well, so it has general practical value (Baker, 1974).

The first notable person to start working on the scheduling problem was Henry Gantt in the early 1900's. Various forms of the Gantt chart that he developed are still used today to represent schedules by scheduling software systems. The first papers on scheduling did not appear until the 1950's and they were authored by scheduling pioneers such as S.M. Johnson, W.E. Smith, and J.R. Jackson (Pinedo, 2002). Since that time a great deal of literature has been devoted to scheduling research. Within scheduling, there are infinite numbers of different problem situations that can be studied. The two main categories are dynamic and static schedules. Dynamic scheduling involves systems that are constantly changing, new jobs can enter or jobs can leave the scheduling environment. In static scheduling systems, the available resources and the number of jobs are known and fixed. Scheduling systems can be further broken down into deterministic and stochastic. In the static, deterministic environment, the number of jobs is fixed and all job data are known, such as processing times. In the static, stochastic environment, the number of jobs is still fixed, but the job data may not be known with certainty. This paper deals with the deterministic static scheduling environment, which will be discussed further. The deterministic static scheduling complexity hierarchy is listed in Figure 1. 


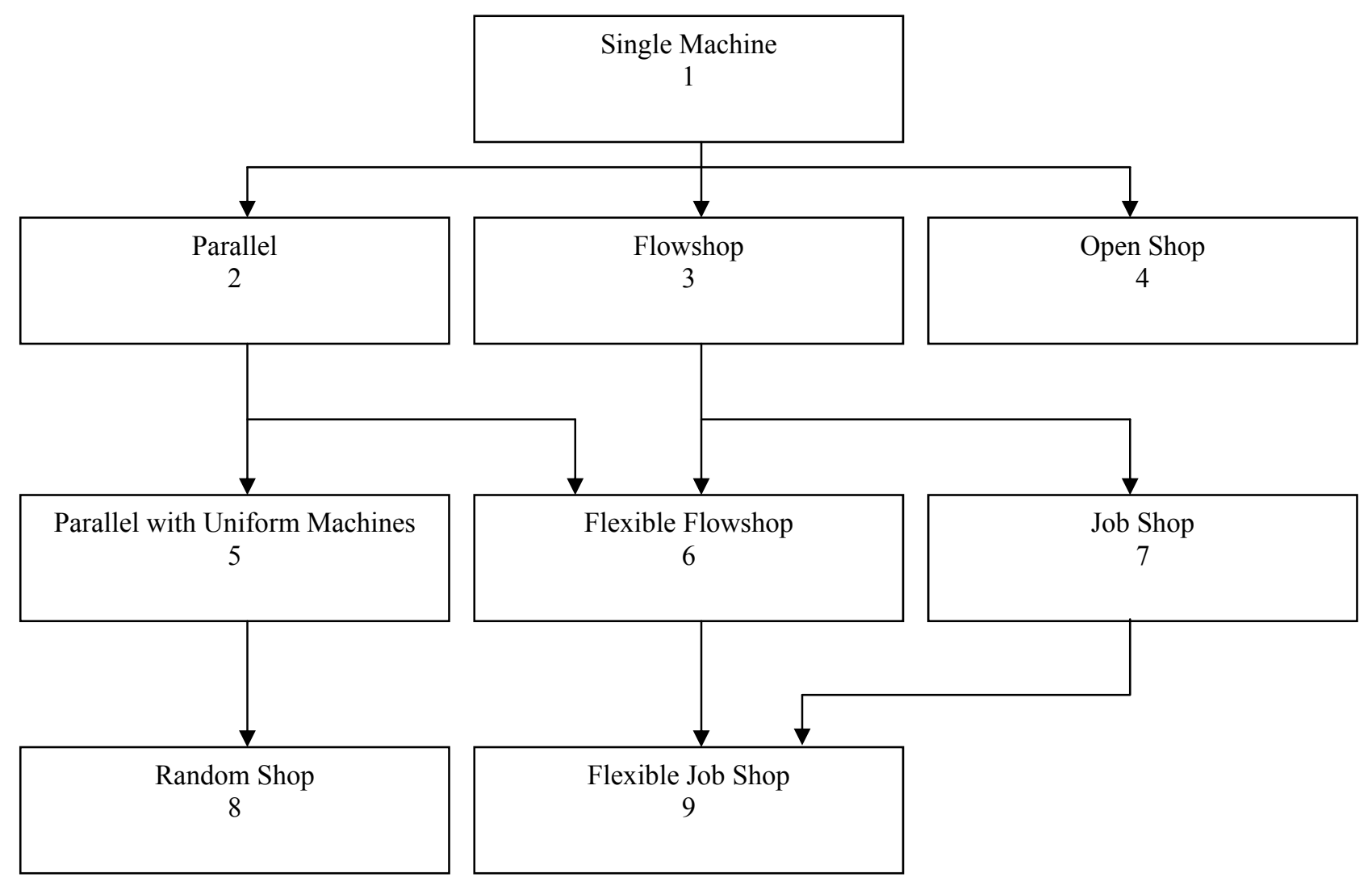

Figure 1.1 Complexity Hierarchy of Deterministic Scheduling Environment (Pinedo, 2002)

(1) The single machine environment is the simplest of all forms, each job $j$ must be processed on one machine.

(2) The parallel machine environment involves one stage where there are multiple identical machines, and each job $j$ must be processed on one of the machines. 
(3) In the flowshop there are $m$ machines arranged in a series and each job $j$ must be processed on each machine and all jobs must follow the same route. Usually jobs are assumed to move through the system in a first in first out manner.

(4) In the open shop each job $j$ must be processed on each of $m$ machines, but some of the processing times may be zero. Also there are no restrictions on the routing of the jobs and each job may have a different route.

(5) The parallel machine environment with uniform machines consists of $m$ machines in parallel, but with different speeds. The processing time depends on which machine the job is processed on and is calculated by dividing the processing time by the machine speed.

(6) The flexible flowshop is a combination of the flowshop and the parallel environment. There is a number of stages in series with each stage having one or more identical machines in parallel. Each job $j$ must be processed on exactly one machine at each stage and the queues may or may not operate on the first in first out or last in first out basis.

(7) A job shop has $m$ machines and each job $j$ has a predetermined route to follow. In some cases a job may visit a machine more than once.

(8) The random shop is very similar to the parallel machine environment with uniform machines. In the random shop there are $m$ machines in parallel with varying speeds, but the machine speeds may be different for each job.

(9) The flexible job shop is a combination of the job shop and parallel machine environments. The flexible job shop consists of work stations with a number of 
identical machines in parallel at each station. Each job has its predetermined route and it must visit one machine at each work station on its route.

Flexible flowshops are a common occurrence in many industries. If the processing times at one stage dominate others, then it is common to add another machine. If changes in demand occur, new machines may be purchased over time and have varying speeds. When the machines or resources are costly, the older versions may not be discarded because they still have some value, even if they are not as fast as the newer models.

Most scheduling problems studied in the literature consider setup times to be included in the processing times. This can affect the quality of the solution if setup times differ based on the preceding jobs. Setup times usually consist of preparing tools, setting jigs, setting fixtures, and positioning the job. Since some jobs may be similar in the work they require, it may require smaller time for set up between these jobs and vice versa. When jobs are waiting for setup, no value is added and in some instances the setup time is directly related to cost, so if the setup times are not properly accounted, time and money could be wasted (Allahverdi, 1999).

This research studies a combination of the flowshop and the parallel shop with uniform machines. The problem considers a flexible flowshop with uniform machines, sequence dependent setup times and limited buffers with the objective of minimizing the makespan. This problem is strongly NP hard (Pinedo, 2002), which means that it belongs to a class of problems that are still NP hard even when all numbers in the input are bounded by some polynomial in the length of the input 
(National Institute of Standards and Technology $<$ http://www.nist.gov $>$ ). As a result finding optimal solutions is not practical for large problems. A construction heuristic will be developed based on the flowshop scheduling algorithm of Nawaz et al. (1983). This algorithm uses a priority list that gives preference to jobs with larger processing times and looks at $[n(n+1) / 2]-1$ solutions instead of all $n$ ! solutions; where $n$ equals the number of jobs. This drastically reduces the number of possible solutions considered; for example, for a problem consisting of 5 jobs there are 120 possible solutions, but this algorithm will only look at 14 solutions. After the initial solution is constructed a simulated annealing algorithm will be used to improve the initial solution.

In this research, "job" refers to a product, "machine" refers to a resource that can perform any one of a number of tasks, and "stage" refers to a set of one or more machines in a group that can all perform the same tasks. The jobs need to be processed at every stage in chronological order, and they can be processed on any machine at any given stage. Each stage may have one or more machines and the machines may have differing speeds. 


\section{Literature Review}

\subsection{Flowshop Scheduling Overview}

Since the pioneering work of Johnson (1954), many researchers have studied various forms of the flowshop scheduling problem. In an article by Dudek et al. (1992), a review is done of flowshop scheduling and it is concluded that the flowshop scheduling problem is a mathematically challenging problem. Some have tried exact approaches such as branch and bound or mixed integer programming. These approaches are useful in understanding the problem structure, but are only practical in solving very small problems. The majority of the work on flowshop scheduling has been devoted to heuristics. The heuristics are designed to find optimal or near optimal solutions in a reasonable time period. Taillard (1993) gives data for testing permutation flowshop schedules. Many papers have referenced his article and tested heuristics against the data and lower bounds presented for makespan minimization. Unless otherwise stated, the objective function of each algorithm in this literature review is to minimize the makespan, which is the time period between when the first job starts processing on the first stage to the time when the last job is finished at the final stage. Since the focus of this research is on the static and deterministic scheduling situations, only those will be considered in this review.

The scheduling literature is so vast that any literature search needs to be selective. The theory of sequencing and scheduling is unlimited in problem types. Lawler et al. (1993) presented a survey on deterministic machine scheduling. They discussed complexity and approximation algorithms for problems involving single machine, parallel machines, open shops, flowshops, and job shops. Sequencing and scheduling is 
concerned with the optimal allocation of resources to activities over time. A machine is a resource that can perform at most one activity at a time, and activities are considered to be jobs that can be worked on by at most one machine at a time (Lawler et al., 1993). Four of the major approaches to dealing with static sequencing are: (1) combinatorial approaches, which change jobs from one permutation to another, (2) general mathematical programming, (3) heuristics, and (4) Monte Carlo sampling (Day and Hottenstein, 1970).

The flexible flowshop is a case of the flowshop where at each stage there may be one or more machines. When there is more than one machine at a stage, three major issues need to be considered (1) which machines will process which jobs, (2) in which order the machines will process the jobs, and (3) how to decide if the schedule is good or not.

There can be many reasons to add machines at a stage such as to increase throughput and reduce the problem of a bottleneck. Also, adding another machine may increase the system reliability and flexibility. If machines are added at different time periods, they may have different speeds resulting in what is termed as uniform machines (Cheng, T., 1990). Linn and Zhang (1999) presented a survey on the flexible flowshop. They stated that most of the research can be grouped into three main categories: 2-stage, 3-stage, and $>3$-stage. There is not much research reported in the literature on uniform machines which considers setup times. In most of the flowshop scheduling research, the setup times are assumed to be included in the processing times, but in reality that may not be the case. When a machine changes from processing one part to another of a different type, the setup times can be $20 \%-40 \%$ of the processing time. Allahverdi et al. (1999) presented a review of scheduling research involving setup considerations. Setup may be 
needed in obtaining tools, positioning wip, returning tool, cleanup, adjusting tools, setting fixtures, and inspection. Two major types of setups are sequence-dependent and sequence-independent. For this research we will be concerned with the first case.

This literature review is not designed to be an in-depth study of the entire field of scheduling and sequencing research. In order to get a better overview, refer to the articles mentioned in this section. This research is mainly focused on the flexible flowshop with buffers, sequence dependent setup times, and uniform machines. Also an overview of simulated annealing is presented in the last section.

\subsection{Exact Solution Methods}

\subsubsection{Flowshop}

The two machine flowshop can easily be solved by Johnson's algorithm (1954). However, branch and bound and dynamic programming have been used to solve some special cases of the two machine flowshop problem. Corwin et al. (1974) presented a dynamic programming formulation for the two-machine scheduling problem with sequence dependent setup times at one stage. When the sequence dependent setup times are at the second stage and there are less than fourteen jobs, dynamic programming is preferred over branch and bound. Agnetis et al. (1998) used a branch-and-bound algorithm in the two machine flowshop, where the input buffer of the second machine is limited. This algorithm can compute optimal or suboptimal solutions for up to 40 jobs. Other than certain special cases, the flowshop with three or more machines is considered to be NP-Hard (Garey, 1976 and Koulamas, 1998). Ignall and Schrage (1965) and Lee, C. et al. (1993) presented branch and bound techniques for the three machine flowshop 
problem. Branch and bound algorithms have also been developed for the multiple machine flowshop, where the number of machines can be greater than three. Carlier et al. (1996) presented two branch and bound algorithms for the m-machine permutation flowshop. Both methods use the depth first strategy. The first method could not solve problems with more than 30 jobs, while the second method could solve up to 50 jobs. Rios-Mercado and Bard (1999A) presented a branch and bound algorithm for the permutation flowshop problem with sequence dependent setup times. The setup times were asymmetric, and lower bounds were calculated by relaxing the problem and reducing it to the two machine case. An upper bound was found by using GRASP, which is a heuristic developed by the authors. The setup times used are usually between $20 \%$ $40 \%$ of the processing times. Also, the brand and bound procedure permits a partial enumeration search procedure that can calculate approximate solutions.

There are other special cases of the flowshop scheduling problem that have special structures which allow them to be modeled as a traveling salesman problem (TSP). Gupta, J.N.D. (1976) looked at the flowshop scheduling problem with no intermediate storage and uninterrupted flow. The objective was to minimize the weighted sum of idle times on machines. The problem was modeled as a TSP and solved using TSP techniques. Gupta, J.N.D. (1986), also modeled the uninterrupted flowshop with sequence dependent setup times as a TSP. 


\subsubsection{Flexible Flowshop Exact Methods}

Arthanari et al. (1971) presented a branch and bound algorithm to optimally solve the special case of the two-stage flexible flowshop (FFS) where there are multiple machines at the first stage and only one machine at the second. Brah and Hunsucker (1991) also presented a branch and bound algorithm for the general flexible flowshop with multiple stages. This algorithm allows for machine idle time and calculates lower bounds based on jobs, machines, and a composite of both. This enumeration method was originally used for parallel machines, but has been modified to fit the flexible flowshop. It optimally solves problems with 4-8 jobs, 2-5 stages, and 2-3 machines at each stage. Azizoglu et al. (2001) presented a branch and bound algorithm to solve the flexible flowshop where the solutions are not restricted to permutation schedules. They used Brah and Hunsucker's (1991) branching scheme and tested the proposed algorithm on two data sets, one with small processing times and one with large. The algorithm works only for small and medium sized problems. Moursli (1995) proposed a branch and bound approach to solve the multistage flexible flowshop problem with multiple machines at each stage. He also presented three improvements to Brah and Hunsucker's algorithm with three new lower bounds. Portman et al. (1998) presented a branch-and-bound algorithm crossed with genetic algorithms (GA). They also made improvements to Brah and Hunsucker's (1991) lower bound. They used several heuristics to calculate initial upper bounds, and then GA to improve the search value of the upper bound. By adding GA, the proof of the optimal solution is found easier and the optimal solution is found more often. Rajendran et al. (1992) presented a branch and bound algorithm for the parallel machine flowshop where only permutation schedules are considered. Vignier et 
al. (1996) presented a branch and bound approach to minimize the total completion time in a k-stage flexible flowshop. The upper bound is found by using the shortest processing time.

Sawik (2002) presented a mixed integer programming approach for the flexible flowshop. There were limited buffers viewed as machines with zero processing times. Also in Sawik (2000), two mixed integer programs were presented for the FFS where one case has buffers and the other does not. Aghezzaf et al. (1998) presented a mixed integer linear programming model for the flexible flowshop with product and machine dependent setup times, but it is feasible only for small problems.

\subsection{Heuristic Methods}

\subsubsection{Flowshop}

\subsubsection{Classic Flowshop Methods}

Since the pioneer work of Johnson in 1954 to minimize makespan in flowshop sequencing, many have tried to develop algorithms that can solve the multiple machine flowshop with the simplicity that his algorithm solved the two machine case. There are four main classic heuristics that have been studied, referenced, used in comparison, and used in constructing new algorithms by many researchers. All of the classic heuristics use permutation schedules. The first is Palmer (1965) who presented a heuristic that gives priority to jobs whose processing time changes from short to long. The slope order produces schedules fairly close to optimal. The second classic algorithm CDS was developed by Campbell, Dudek, and Smith (1970). This procedure can be applied by hand and generates $(\mathrm{m}-1)$ schedule sequences, where $m$ is the total number of machines. 
It outperforms Palmer's, which produces only one sequence. The algorithm can solve for 3-60 jobs and 3-30 machines, or even larger problems. The third classic algorithm was given by Dannenbring (1977). In his paper, he compares eleven flowshop heuristics, three of which were previously unreported. Heuristics from previous literature included Palmer and CDS, but the new heuristic he developed and called Rapid Access with Extensive Search (RAES) performed the best. Problems were solved with up to 50 jobs and 50 machines. In the larger problems, RAES found the best solution in $71.25 \%$ of the problems, while the next best rule found the best solution in $18.13 \%$ of the problems. The algorithm also has a neighborhood search strategy that changes neighbors until there is no improvement. Even though Dannenbring's algorithm worked well, the best of the classic algorithms is the fourth, which was presented by Nawaz, Enscore, and Ham (1983) (NEH). Taillard (1990) and others came to the same conclusion when comparing the classic heuristics. This algorithm is still considered to be one of the best methods of constructing initial solution (Koulamas, 1998). Numerous researchers still use NEH in their construction algorithms before applying an improvement heuristic. NEH is superior to CDS and all other algorithms developed up to 1983. It uses the assumption that jobs with more processing times should get a higher priority. The only way CDS would outperform NEH is if the number of machines greatly outnumbers the number of jobs (Turner and Booth, 1987).

\subsubsection{Recent Flowshop Heuristics}

Hundal et al. (1988) extended Palmer's algorithm and allowed it to generate three sequences instead of one. They combined CDS with Palmer and added a pairwise 
exchange search strategy. Using these extensions improves the solution, but also adds computation time. Koulamas (1998) presented a construction heuristic for the flowshop scheduling problem. In this heuristic, non-permutation as well as permutation schedules are considered. Koulamas's heuristic performed as well as NEH when the optimal schedule is a permutation and better when the optimal schedule is not a permutation. Ho et al. (1991) developed a heuristic designed to reduce gaps between operations in solutions generated by other heuristics. The algorithm was compared to CDS, Palmer, Gupta, Dannenbring, Hundal and Rajgopal's (1988). For minimizing makespan, Ho, CDS, and Hundal and Rajgopal's worked the best. Sarin et al. (1993) developed a heuristic, that attempts to minimize the idle time on the last machine. Only permutation schedules were considered. When compared with NEH (1983), NEH worked better for small problems, but Sarin's heuristic dominated for larger problems.

Chen et al. (1996) presented a heuristic for the three machine flowshop. The heuristic is based on Johnson's algorithm and has a worst case performance ratio that is better than 2. Lee, C. et al. (1993) also presented heuristics and their error bounds for the three machine flowshop problem. Barman (1998) looked at combining 3 different priority rules for the three-stage flowshop where there are two machines at each stage. The objectives were to minimize mean lateness, mean tardiness, maximum tardiness, and percent tardy. The results showed that the combination of rules work better than using any one rule by itself.

Carlier (1982) presented a unique algorithm that gives the bottleneck stage priority over all others. The jobs are scheduled in a way that minimizes the time spent at the bottleneck stage, also the algorithm considers release and processing times. Egin et 
al. (2004) proposed an artificial immune system (AIS) that is similar to neural networks. Wang et al. (1997) presented two heuristics, the first heuristic reduces machine idle time, and the second reduces machine idle time and job queue times.

\subsubsection{Flowshop with Setup/Buffers}

Other special cases of flowshop scheduling are those having setup times, limited or no buffers, or uniform machines. In general flowshop scheduling, the processing times include all setup times, the buffers are considered to be unlimited, and all machines are identical (i.e. have the same speed). Sule (1982) looked at scheduling jobs on a two machine flowshop. The processing time is separated into setup, processing, and removal times. Also it further categorizes operations into inside and outside processing. Gupta, J.N.D. et al. (1987) presented a heuristic for flowshop scheduling with sequencedependent additive setup times. The approximate solution can yield optimal schedules for the two machine case. The heuristic produced better solutions than Sule's algorithm.

Simmons (1992) presented four heuristics for the flowshop sequencing problem with sequence dependent setup times. The results showed that sophistication does not necessarily lead to better performance. One of the heuristics (TOTAL) uses the sum of processing and setup times, where another (SETUP) just works with the sum of setup times. TOTAL performed the best. Rios-Mercado and Bard (1998) presented two heuristics for the flowshop scheduling problem with sequence dependent setup times, where only permutation schedules are considered. The first heuristic was an extension of the NEH flowshop heuristic, and the second was a greedy randomized adaptive search procedure (GRASP). GRASP has a construction and improvement phase. Both 
heuristics were compared with Simmons's (1992) (SETUP) heuristic using two data sets.

For the first data set, a traveling salesman heuristic (TSP) worked best for a small number of machines, but (GRASP) worked better for a large number of machines. For the second data set SETUP worked the best. Rios-Mercado and Bard (1999B) presented another heuristic for solving the permutation flowshop problem with setup times. The procedure transformed the problem into a Traveling Salesman Problem (TSP). Large setup times and makespan are factors that penalize the objective function. The setup times used were asymmetric and equal to $20 \%-40 \%$ of the processing time. The heuristic worked better when the number of machines was small.

Allahverdi (2000) looked at minimizing the mean flow time in the two-machine flowshop with sequence independent setup times. Three heuristics were given with an overall average error of $0.7 \%$ of the optimal. Also, optimal solutions were found for two special cases. Experiments were performed with job sets of 10, 15, 20, 25, 30, and 35. The flowshop with sequence dependent setup times and limited buffers was studied by Gupta, J.N.D. (1986), but no experiments were performed.

King and Spachis (1980) looked at existing and new heuristics to solve the flowshop problem. The new heuristics studied the permutation, no-wait flowshop. The no-wait flowshop is reducible to the asymmetric traveling salesman problem. For no passing, the least total weighted between-jobs delay (LBJD) heuristic performed well. For the no-wait problem, the minimum covering level, the maximum left shift savings, and LBJD heuristics worked well. Details of these heuristics can be found in King and Spachis (1980). 


\subsubsection{Flowshop Heuristics Using Meta-heuristics}

Most of the recent work in flowshop scheduling has been devoted to the combinatorial approach and the use of meta-heuristics. The three traditional metaheuristics used in the literature are Tabu Search (TS), Genetic Algorithms (GA), and Simulated Annealing (SA). SA and TS have both been found to work well for the permutation flowshop problem. In this research, SA will be used, so the literature covered will focus mainly on SA with some brief mention of the other methods. Also, it should be noted that a new heuristic, called Ant Systems has recently been applied to the permutation flowshop problem with comparable results to those of SA and TS.

Osman and Potts (1989) presented a simulated annealing (SA) heuristic for the permutation flowshop scheduling problem. To search the neighborhood, a non-adjacent interchange and a forward/backward shift system are used. Four different SA algorithms were used and they perform better than NEH, but require more time. Ogbu et al. (1990B) presented a different simulated annealing heuristic for the flowshop scheduling problem. This heuristic used an acceptance probability that is the same for all iterations. The paper states the importance of reducing the temperature slowly. Also the heuristic used a last improvement technique that is different from Osman and Potts (1989) which used first improvement. For this heuristic, the insertion/shift perturbation scheme worked better than the interchange/pairwise. The paper also stated that for the n-job m-machine flowshop, SA outperforms all other heuristics. Ogbu and Smith (1990A) also used simulated annealing to solve the flowshop scheduling problem. The probability of acceptance of an inferior solution is independent of the change in function value. Two perturbation schemes are used; one uses pairwise exchange and the other uses job 
insertion. Insertion seemed to work better in the larger problems. In theory, the choice of a starting solution will not matter, but practical experience showed that a good starting solution leads to more efficient convergence. The initial starting solutions of Palmer and Dannenbring were used here. Two sets of problems were considered, the small set had 7 jobs and 5 machines, and the large has 15 jobs and 10 machines. The small problems were solved by branch and bound for comparison, and for the large problems the solutions were compared by using the best solution found. The proposed heuristic found better solutions in a set time period than traditional approaches. Kouvelis et al. (1992) also developed a simulated annealing procedure. The initial solution is randomly generated, but the article states that initial solutions that exploit specific problem structure will get better final solutions. Ishibuchi et al. (1995) developed two simulated annealing algorithms with modifications to the generation mechanism. The generation mechanism is designed so the choice of cooling schedule will not affect the quality of the solution; it works with a pool of possible solutions. They found that Tabu search is slightly inferior to their algorithm. The proposed algorithm was also compared to the SA algorithm presented by Osman and Potts (1989), and it was found that the proposed is better for the 20 job problem and Osman and Potts algorithm was better for the 50 job problem. Zegordi et al. (1995) presented a heuristic that combines simulated annealing with problem specific knowledge. Johnson's rule is used to develop a move desirability index. The proposed algorithm was compared with NEH, CDS, and Osman and Potts. Osman and Potts performed better on larger problems, but the proposed algorithm was better on smaller problems. Low (2004) developed a simulated annealing algorithm that uses a modified NEH algorithm as the initial starting solution. Three different 
neighborhood search schemes are used: adjacent pairwise exchange, general pairwise exchange, and insertion. General pairwise exchange and insertion can yield good solutions, but they are computationally costly. Adjacent pairwise exchange is considered to be not as computationally costly. The algorithm was tested using Taillard's (1993) data and it was found that the proposed SA with an initial starting solution of SPT/FCFS worked the best. Tian et al. (1999) used simulated annealing to solve combinatorial optimization problems with permutation properties. The problems studied are traveling salesperson, flowshop, and quadratic assignment. Six different perturbation schemes were used for each problem and the results were presented. For the symmetric traveling salesperson, block reversal worked best. For the flowshop, two job random exchange and block insertion were the best performers.

Widmer and Hertz (1989) presented a two phase heuristic where the first phase used a construction heuristic based on an analogy of the problem with the traveling salesperson problem. In the second phase, the initial solution is improved using tabu search. Their algorithm was compared with NEH, CDS, and Dannenbring's RA, and the proposed algorithm produced the best sequence $80 \%$ of the time. Taillard (1990) presented a tabu search heuristic that was compared to NEH and shown to be superior. Ying et al. (2004) presented an ant systems approach to the permutation flowshop problem. The test data of Taillard (1993) were used. Ant systems can get good solutions with reasonable computation time. The results showed that the proposed ant system heuristic is on average minimally better than SA.

Weng (2000) used a modified NEH algorithm to solve the flowshop scheduling problem with limited buffers. NEH is used to generate the initial permutation schedule, 
and then tabu search (TS) is used to improve the solution. The objective function is to minimize the mean job flow time. TS was shown to improve the solution, and buffers greater than four are not needed. As the numbers of machines or buffers increase, the TS improvement decreases.

Parathasarathy et al. (1997) used simulated annealing to solve the flowshop scheduling problem with sequence dependent setup times and the objective of minimizing the mean weighted tardiness. A case study was carried out in a drill bit manufacturing industry. The simulated annealing heuristic was compared to tabu search and out-performed it $70 \%$ of the time. Also the proposed algorithm solved the 95 job problem in 3 hours while tabu search took 12 hours. Norman (1999) presented a tabu search heuristic for the flowshop problem with limited buffers and sequence dependent setup times. NEH was used as the construction algorithm. Also, another heuristic was developed using greedy improvement, but tabu search works much better. Good lower bounds are difficult to establish because of the buffers and the asymmetric setup times.

\subsubsection{Flowshop Scheduling Using Combination of Meta- heuristics}

A new method of solving the flowshop problem is to combine two metaheuristics; most of this has been done by combining SA and GA. By combining these two heuristics, good solutions can be obtained. Wang et al. (2003B) presented a hybrid heuristic for the flowshop scheduling problem. The algorithm used NEH, GA, and SA. The heuristic outperformed NEH and was comparable to TS, SA, and GA. Allahverdi et al. (2004) presented a hybrid simulated annealing and genetic algorithms heuristic to solve the no-wait flowshop. The objective was to minimize the weighted sum of 
makespan and maximum lateness. Nearcchou (2004A) used a hybrid simulated annealing algorithm to solve the flowshop problem. The heuristic has the basic structure of SA with some aspects borrowed from genetic algorithms and local search techniques. A random exchange perturbation scheme is used. This heuristic worked with a population of possible solutions at each iteration. The solutions obtained are comparable to the best available in the literature. Nearcchou (2004B) presented a hybrid simulated annealing algorithm to solve the permutation flowshop problem. The simulated annealing algorithm is crossed with genetic algorithms. Initial solutions are randomly generated and a shift perturbation scheme is used. The proposed algorithm can find makespans faster than other known meta-heuristics.

\subsection{Flexible Flowshop Heuristics}

Finding the minimum makespan in a flexible flowshop problem is considered to be NP-Hard (Hoogeveen et al., 1996). Therefore most of the research in FFS scheduling has revolved around heuristic methods. Since heuristics are approximation methods and are not likely to find the optimal solution, a method of testing their performance is needed. There are three main methods to test a heuristic, test it against a lower bound, against other heuristics for the same problem, or against the best solution found. Santos et al. (1995) presented global lower bounds for makespan minimization on the flexible flowshop scheduling problem. The purpose was to give a bench-mark to test the quality of heuristics. The optimal makespan was predicted $38 \%$ of the time and the average relative error was $4.6 \%$. Also in $85 \%$ of the 653 problems tested, the bounds were within $10 \%$ of the optimal solution. 


\subsubsection{Two-Stage Flexible Flowshop}

Gupta, J.N.D. (1988) showed that the two-stage flowshop where there are multiple identical machines at each stage is NP-Complete. Gupta, J.N.D. et al. (1997) presented a heuristic to solve the two-stage flexible flowshop problem with multiple machines at the first stage and one machine at the second stage. The heuristic uses Johnson's rule to create a list of jobs, then this list is used in one of five heuristics. Also a steepest descent strategy is used to find local optima from the heuristic's solution. Lee et al. (1998) presented two heuristics for the same problem, one is a look ahead and the

other a look behind. Also, a dynamic programming algorithm was developed for the two stage problem with two machines at each stage. Oi (1996) developed four heuristics for the same problem. He assumed that the manufacturing is done at the first stage and assembly is done at the second stage. The objective was to minimize the sum of weighted customer lead times. Only permutation schedules were considered. The four heuristics are Lagrangian relaxation, greedy search, total weighted shortest processing time, and one that is simply tardiness based. Chen (1995) addressed the two-stage problem with parallel machines at one stage and a single machine at the other. Oguz et al. (1997) looked at the two-stage flexible flowshop problem with different machines at the first stage and one common machine at the second stage. He developed a heuristic based on Johnson's rule which was found to be effective.

Narasimham et al. (1984) looked at scheduling rules in a two-stage flexible flowshop with one machine at the first stage and two machines at the second. The objective was to minimize the sum of machine idleness and in-process waiting time at the second stage. Cumulative minimum deviation worked the best, minimum deviation 
(MD) second, and SPT and LPT were last. Gupta, J.N.D. et al. (1991) looked at scheduling jobs in two-stage flexible flowshop with one machine at the first stage and multiple machines at the second. They developed an algorithm that can be used to find approximate solutions or to increase the efficiency of a branch and bound algorithm. They had two objectives; the first was to minimize makespan and the second to minimize the number of machines. This problem was also considered by Tsubone et al. (1996). In Tsubone et al., the first stage used the shortest processing time, longest processing time, RATIO, and a Modified Johnson's rule. The second stage simply used first come first serve. The RATIO rule worked best for makespan minimization. Li (1997) presented a backward and a forward scheduling heuristic for the same problem. The heuristics performed better than shortest processing time (SPT) and longest processing time (LPT). A Pratt and Whitney Blade production line was the basis for this scheduling problem. The backward algorithm performed better than the forward. Huang (1998) studied a similar problem, but the first stage had one machine and the second stage had multiple uniform machines. In his paper, the parts are grouped into families with major and minor setup times that are independent of the machine speeds. The problem was examined by using two heuristics and eight sequencing rules. This scheduling problem also came from a Pratt and Whitney Blade manufacturing facility. Average results were between 20\% and $60 \%$ above the lower bounds. Riane et al. (1998) developed two heuristics for the two stage hybrid flowshop with one machine in the first stage and two dedicated machines in the second stage. The first heuristic was based on dynamic programming, while the second used a construction heuristic combined with a greedy algorithm. The dynamic programming approach worked best. For the same problem, Riane et al. (2002) 
proposed two heuristics that were found to be efficient. A dynamic programming approach was also used for problems with less than 15 jobs.

Lee et al. (1994) presented a heuristic for solving the general two-stage flexible flowshop. Five lower bounds were used to test the algorithm and an additional heuristic was developed for the case with more than 2 stages. As the number of machines increases, the problem becomes harder. Guinet et al. (1996) looked at scheduling of twostage flexible flowshops with unlimited storage. A MIP formulation was presented and lower bounds were calculated. A sequence first and allocation second heuristic was developed that uses Johnson's rule and different combinations of seven other rules developed by other authors to create a priority list, then the jobs are scheduled using the priority list. Lin et al. (2003) studied the two-stage flexible flowshop with sequence dependent setup times at stage one and dedicated machines at the second stage. This problem came from a label sticker manufacturing company. A tabu search algorithm was used in the first stage and a first in first out (FIFO) algorithm was used in the second stage. The objective was to minimize the maximum weighted tardiness. The heuristic performed well and was to be implemented at the company for which it was designed. Narasimham et al. (1987) tested three scheduling rules for the two-stage flexible flowshop. The rules tested were shortest processing time (SPT), longest processing time (LPT), and minimum deviation. The problem was modeled after a scheduling problem in the Schlitz Brewing Company. The objective function has multiple criteria such as minimizing total cost of order waiting, machine idle time, makespan, and average completion time. Generalized cumulative minimum deviation rule (GCMD) worked the best. Soewandi et al. (2003) developed three heuristics for scheduling in the two-stage 
flexible flowshop with uniform machines at each stage. Some of the rules used are the earliest finished machine (EFM) and latest start machine (LSM). Uetake et al. (1995) looked at the two-stage FFS with one machine at the first stage and multiple different machines at the second stage. The objective was to minimize makespan and maximum work in process. This type of system occurs in steel, chemical, and paper industries. The first stage used SPT, LPT, RATIO, Johnson's rule, and the second stage used the first come first serve rule (FCFS). Verma et al. (1999) showed that the two-stage FFS with parallel and uniform machines is NP-Hard. Three different heuristics were presented; earliest completion time (ECTH), fastest available machine heuristic (FAMH), and mixed heuristic (MH). The mixed heuristic is just a combination of the others and tends to perform the best.

\subsubsection{Three-Stage Flexible Flowshop}

Koulamas et al. (2000) presented a linear time algorithm for two-stage and threestage flexible flowshops. The worst case bounds are no worse than those currently available. Soewandi et al. (2001) presented two heuristics for the three-stage problem. Some rules that were used in the formation of the heuristics are Johnson's, first available machine, last busy machine, and modified Johnson's rule.

\subsubsection{General Flexible Flowshop}

Wittrock (1985) looked at scheduling in flexible flow lines. The objective was to maximize throughput and reduce work in process. He considered two scheduling decisions, one is when should a job enter the system, and the other is what should the 
daily product mix be. The buffer works on a first come first serve basis and the heuristic tries to balance workloads. Wittrock (1988) also looked at an adaptable scheduling algorithm to minimize makespan and queueing in the flexible flowshop. The heuristic breaks the problem into three subproblems; machine allocation, sequencing, and timing. No queues are allowed in front of an idle machine, even though sometimes it may improve the solution. Kochhar et al. (1988) studied entry point scheduling. This technique uses local perturbation to obtain schedules within a few percent of optimal. A construction heuristic and random starting solutions were used. They state in their paper that a good starting solution is one that will lead the local search into different areas. The local search techniques used are pairwise exchange and block exchange, both with steepest descent and multiple starting solutions. Ding et al. (1994) presented three heuristics for the flexible flowshop with 1 to 3 machines at each stage. Two of the heuristics are based on Johnson's and CDS algorithms, while the third is based on Gupta (1972). The algorithms based on Johnson's algorithm worked the best. Guinet and Solomon (1996) looked at scheduling jobs in a flexible flowshop with the objective of minimizing the makespan or maximum tardiness. The heuristics used are based on CDS, NEH, and Townsend (1977). NEH worked the best for makespan minimization. Leon et al. (1997) presented a heuristic that was previously used for job shop scheduling, but adapted it for the flexible flowshop. The heuristic uses the shortest processing time rule combined with local search techniques. The local search techniques used are single neighborhood, steepest descent, and first improvement. Brah and Lou (1999) compared five heuristics for the FFS with the objective of minimizing makespan and mean flowtime. The heuristics tested are NEH, HO, CDS, PAM, and CDS2. NEH performed 
the best for makespan. The heuristics were compared using the global lower bounds presented by Santos (1995). Chang (1994) developed an algorithm based on Lagrangian relaxation and minimum cost linear network flow. A rescheduling algorithm is also given. The objectives are to avoid overdue penalty, reduce cost of WIP, and reduce cost of overtime. Jayamohan et al. (2000) looked at minimization of flow time and tardiness of jobs in a flexible flowshop. Two approaches were investigated; one uses the same dispatching rule at all stages while the other uses different rules at different stages. This study found that using one good rule at all stages works best, which contradicts a previous study done by Barman (1998), but his study was on the three stage flowshop. Also, it should be noted that using different rules at different stages adds a great amount of computation time.

\subsubsection{Flexible Flowshop Bottleneck Heuristics}

A few researchers took a different approach to scheduling in the FFS by putting emphasis on the sequence at the bottleneck stage. Adams et al. (1988) presented a shifting bottleneck heuristic (SBH) that is designed for the job shop scheduling problem, but can be applied to the flexible flowshop. It works by scheduling jobs on the bottleneck machine and then re-sequencing the other stages. Their algorithm can solve a ten job, ten machine problem in less than six minutes. Cheng et al. (2001) used the shifting bottleneck heuristic for the FFS in order to minimize the maximum lateness. The heuristic works in two phases, the first schedules $m$ stages one by one successively in descending order of lower bounds, and the second phase re-optimizes each stage. The results showed that optimal or near optimal solutions are found in short time periods. 
Phadnis et al. (2003) presented a progressive bottleneck improvement (PBI) procedure to solve the FFS. Only one job is sequenced at a time and it is sequenced at all stages. The bottle neck stage is identified before each job is scheduled. The heuristic is similar to Adams (1988) shifting bottleneck heuristic. Phadnis's heuristic is simple and produced good solutions. Acero-Domingvez et al. (2004) developed a heuristic based on the theory of constraints by optimizing the bottleneck stage. By optimizing the bottleneck stage the overall system is improved; if other areas are improved they will only achieve local improvement and the entire system may not benefit. The heuristic requires three steps: 1) bottleneck identification, 2) scheduling of jobs at bottleneck stage, and 3) schedule nonbottleneck stages. The algorithm was compared with the Shifting Bottleneck Heuristic and performed comparably. Lee et al. (2004) used a bottleneck focused algorithm in order to minimize total tardiness in a flexible flowshop. The problem in question comes from the printed circuit board industry. The assumptions made include unlimited buffers and that the machines never fail. The algorithm first schedules the bottleneck stage and then schedules the other stages.

\subsubsection{Flexible Flowshop with Setup Times/Buffers}

Another special case of the flexible flowshop sequencing problem is that of having setup times, limited buffers, or both. Kochhar and Morris (1987) presented heuristic methods to solve the flexible flowshop sequencing problem with limited buffers and setup times. The problem was broken into two parts, entry point scheduling and dispatching. The problem was further broken down into three parts; initial sequence, how to manage work in process, and if multiple jobs are available, which ones are 
chosen. The steepest descent neighborhood search technique was used with the perturbation schemes of pairwise and block exchange. A realistic problem of 16 machines was solved in 30 minutes while smaller problems took seconds. Twelve cases were solved and the average performance was $0.5 \%$ from optimal, with the worst case performance about $2 \%$ from optimal. Sawik (1987) developed three heuristics for the flexible flowshop with limited buffers. Beside makespan, the objective function included minimization of work in process holding costs. In two of the heuristics, schedules are period by period and fixed, while the third is capable of making adjustments to the schedule when a buffer becomes full or a shortage to a machine occurs. Sawik (1995) also presented a single pass part-by-part heuristic for the flexible flowshop with no buffers. The schedule is determined only once and run time is short. Khmelnitsky et al. (1997) developed a numerical method and two heuristics to solve the flexible flowshop with partial sequence dependent setup times. Aghezzaf et al. (1998) presented a LP based heuristic for the FFS where setup times are product and machine dependent, the heuristic is based on the duality of linear programming. This algorithm requires a large amount of computation time; at each iteration a trans-shipment problem must be solved. Liu et al. (2000) presented a Lagrangian relaxation based approach for the flexible flowshop with sequence dependent setup times. The objectives were to meet due dates, reduce work in process, and reduce setup. In testing 16 problems, the average difference between the proposed solution and the true optimum was 15\%. Wang et al. (2003A) looked at using a neural network system to solve the flexible flowshop problem. The learning strategy borrows from simulated annealing and lagrangian relaxation. The lower 
bounds used for comparison were obtained from Santos (1995). Neural networks did not provide good solutions in short times when compared to other heuristics.

\subsubsection{Flexible Flowshop Using Meta-heuristics}

This section discusses some of the meta-heuristics that have been applied to the flexible flowshop, with the main focus on simulated annealing. Haouari et al. (1997) used two two-phased heuristics based on simulated annealing and tabu search to solve the two stage flexible flowshop with parallel machines at each stage. To construct an initial solution, the most work remaining rule was used to create a priority list. The objective was to minimize makespan. Tabu search performed just a little better than simulated annealing. Riane et al. (1999) presented a simulated annealing algorithm to solve the flexible flowshop problem where only permutation sequences are considered. The paper emphasizes the importance of temperature reduction; spending too much time at a high temperature wastes time and decreasing the temperature too fast will limit the search to local optima. Negenman (2001) tested a variable depth search method combined with three simulated annealing heuristics and three tabu search heuristics to solve the flexible flowshop. The combination of variable depth search with tabu search performed the best. Loukil et al. (2005) used simulated annealing for multiple-objective scheduling for the one-machine, parallel machine, and permutation flowshop problems. Their neighborhood search schemes consisted of random job exchange and job insertion. A random choice of these two was made. 


\subsubsection{Flexible Flowshop with limited buffers Using Meta-heuristics}

Shieh (2003) studied the flexible flowshop with limited buffers. The objective was to minimize the makespan and maximize the machine free time. An exact algorithm was developed using mixed integer programming, and was able to solve only very small problems. It works in two phases; the first minimizes the makespan and the second maximizes the machine free time. A construction algorithm was developed called (MGPAFFS). Then a simulated annealing improvement heuristic was used to improve the construction algorithms solution. Two sets of data were used; one with 30 jobs and the other with 60 jobs. The results show that after the application of SA, the solutions for the small and larger sets were $1.6 \%-28.3 \%$ and $2.2 \%-32.7 \%$ respectively from the lower bounds. Also SA improved the construction heuristics solution by $1.2 \%-4.9 \%$ and $0.9 \%$ $8.9 \%$ for the small and large data sets respectively.

\subsubsection{Flexible Flowshop with Setup Times Using Meta-heuristics}

Low (2005) presented three simulated annealing algorithms for the multistage flowshop with unrelated parallel machines. This problem has independent setup and dependent removal times. A well designed solution generator was used to find the initial solution. Sethanan (2001) looked at the problem of scheduling a flexible flowshop with sequence dependent setup times and uniform machines. A mathematical model was developed to solve small problems and two heuristics were presented to solve larger problems. The first heuristic is a construction heuristic called "flexible flowshop with sequence dependent setup times heuristic" (FFSDSTH). The second heuristic is a tabu search and was used to further improve the solution obtained from the construction 
heuristic. Two lower bounds are calculated; one uses a forward system and the other a backward. The machine waiting time, idle time, and total setup and processing times on the last machine were used to calculate the lower bounds. The results show that FFSDSTH is efficient at finding solutions and that TS improves the solution between $2.95 \%$ and $11.85 \%$.

\subsection{Simulated Annealing}

Metropolis et al. (1953) were the first to look at the properties of annealing in order to solve mathematical problems. Simulated annealing is derived from an analogy between the physical annealing of solids and combinatorial optimization. Physically, it refers to the heating of a substance to the melting point, then lowering the temperature slowly and spending a long time at temperatures close to the freezing point. The ground state of the solid will have a certain structure and the cooling will not obtain a good structure if it is not done properly (Eglese, 1990). Kirkpatirick et al. (1983) were the first to use simulated annealing to solve combinatorial problems. They listed some of the positive aspects of simulated annealing such as: (1) significant improvements over random starting solutions are possible, (2) there are many near optimal solutions, so a stochastic search procedure such as SA should find some, (3) no one of the ground state solutions is any better than the others, so it is not worth it to search for the absolute optimal (Kirkpatirick et al., 1983). One thing that makes simulated annealing and other metaheuristcs different from simple local search procedures is that the algorithm avoids being trapped in a local optimum by sometimes accepting a neighborhood move that increases the value of the solution (Eglese, 1990). Many researchers have shown that 
simulated annealing can find good solutions for scheduling permutation flowshop problems (e.g. Allahverdi et al., 2004, Haouari et al., 1997, Ishibuchi et al., 1995, Kouvelis et al., 1992, Loukil et al., 2005, Low, 2005, Low et al., 2004, Nearchou, 2004A, Negenman, 2001, Ogbu et al., 1990A/B, Osman et al., 1989, Parthasarathy et al., 1997, Riane et al., 1999, Shieh, 2003, Tian et al., 1999, and Zegordi et al., 1995). Simulated Annealing will be used in this study in an effort to improve the initial solution obtained using two construction algorithms. 


\section{Problem Statement and Objectives of Research}

\subsection{Problem Statement}

This research addresses the flexible flowshop with sequence dependent setup times, uniform machines, and limited buffers. There are one or more machines at each stage, and more than two stages. The machines at each stage have different processing speeds and the first machine is considered to be the fastest. Each job has a base processing time that is the time to be processed on the fastest machine. The processing times for the other jobs are found by dividing the base processing times by the machine speed. There are unlimited buffers before the first stage and after the last stage. For the stages in between, the buffers are assumed to have limited capacity. If a buffer becomes full, it will block machines at the previous stage until space becomes available in the buffer. The environment is deterministic; that is the number of jobs and all of their data are known in advance and fixed. The number of stages and machines at each stage are known in advance and fixed. Jobs are considered to be tasks and must be processed on machines, in each stage, in technological order. Machines are considered to be resources that perform tasks. The objective is to minimize the makespan, which is the completion time of the last job at the last stage. The flexible flowshop with limited buffers is shown in Figure 3.1.

The standard flowshop with more than two machines with the objective of minimizing makespan is considered to be NP-Hard in the strong sense (Pinedo 2002). This problem is considerably more complex because it adds uniform parallel machines at each stage, limited buffers, and sequence dependent setup times. Some research has been done on the two stage flexible flowshops, but for greater than two stages the research is 
scarce. No research could be found in the literature that attempts to solve the flexible flowshop with sequence depended setup times, uniform machines, and limited buffers.

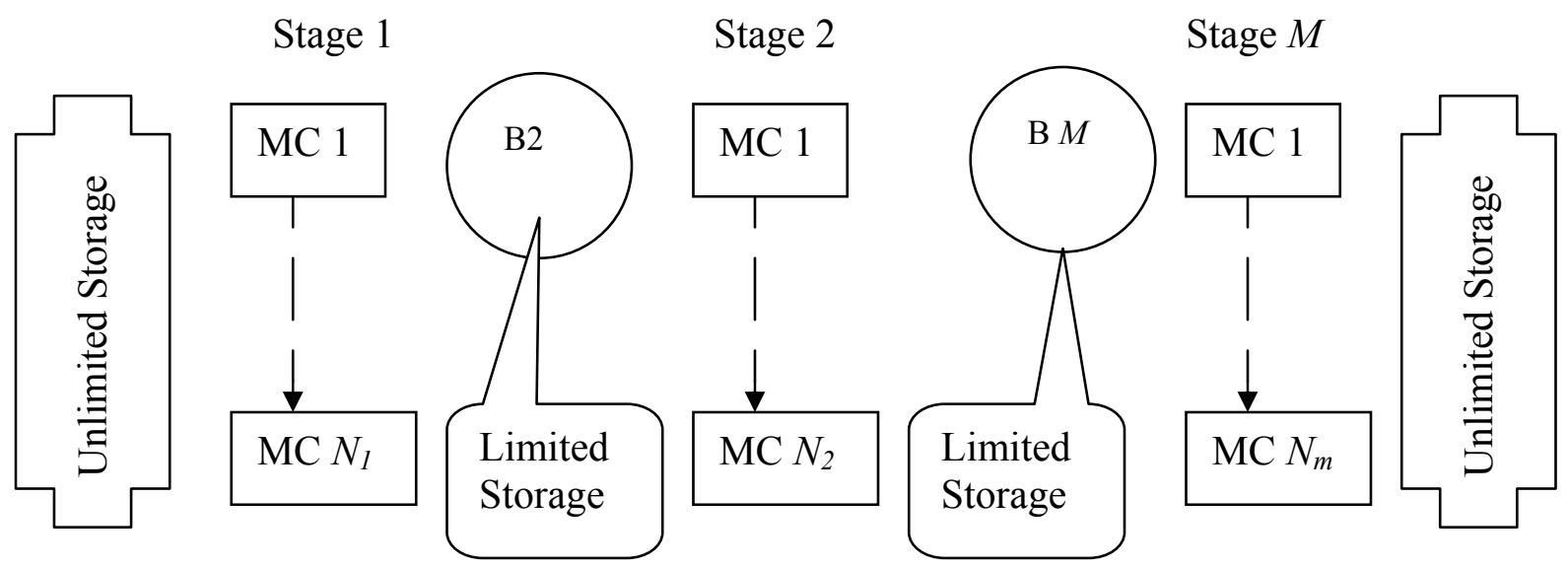

Figure 3.1 Flexible Flowshop with limited Buffers

\subsection{Problem Assumptions}

Because scheduling problems have a vast amount of variation, the following assumptions are made for the problem under consideration.

1. The number of jobs to be scheduled and their processing times on each machine at each stage is known in advance and fixed.

2. The number of stages and the machine configuration at each stage are known in advance and fixed.

3. Preemption is not allowed.

4. Once a job has started processing, it must be completely finished before it can move to the next stage.

5. No job splitting is allowed, a job must be processed on one and only one machine at each stage.

6. All jobs are ready to begin processing at time period 0 . 
7. Jobs may or may not be scheduled in the same order at each stage, i.e. job passing is allowed.

8. Setup times are sequence dependent. There is also a setup time associated with startup.

9. Setup times are the same for all machines within each stage regardless of which machine the job is to be processed on.

10. The setup times uniformly range from $20 \%$ to $40 \%$ of the job processing time.

11. The machines at each stage are uniform. The first machine is considered to be the fastest machine with a speed of $100 \%$, the other machines have either the same or a slower speed than the first (e.g., $60 \%, 50 \%$, or $40 \%$ ). The processing time is found by dividing the base processing time by the machine speed.

12. There are unlimited buffers before the first stage and after the last stage, but between stages the buffers are limited. The buffer is numbered the same as the stage that it feeds into.

13. Machine blocking does occur, so when a job is finished processing, it cannot leave the machine unless there is room on a machine at the next stage or the buffer for the next stage has an open space.

14. There are no due dates associated with the jobs and the objective is to minimize the makespan. 


\subsection{Objectives and Methodology of Research}

There are four main objectives to this research:

1. To develop a mathematical model with the objective of minimizing the makespan for the flexible flowshop with sequence dependent setup times, uniform machines, and limited buffers.

2. Since this problem is considered strongly NP-Hard, develop heuristics to solve this problem. First two construction algorithms are developed to obtain solutions.

3. After the construction algorithm is developed; use a simulated annealing heuristic to improve the solutions obtained from the construction algorithms.

4. Since this problem is very complex even small problems may not be able to be solved optimally in reasonable time. A lower bound will be developed and used to test the performance of the heuristic. 


\section{Mathematical Model}

Only small problems can be solved optimally, and even with small problems the computation time is great. Even though only small problems can be solved by using the mathematical model, there are still benefits to developing and studying it. Developing the mathematical model helps one better understand the problem complexity and structure. The difficulties that will arise in choosing a heuristic will usually come to the surface after working through the mathematical model. The model hence has two main benefits, better understanding of the problem and foreshadowing of issues with heuristic development. Variables used in the mathematical model are listed in Table 4.1.

Table 4-1 Mathematical Model Nomenclature

\begin{tabular}{|l|c|l|}
\hline Variable & Notation & Definition \\
\hline \multirow{4}{*}{ Parameters } & $j, i$ & Job index. \\
\cline { 2 - 3 } & $J$ & Total number of jobs. \\
\cline { 2 - 3 } & $s$ & Stage index. \\
\cline { 2 - 3 } & $m$ & Machine index. \\
\cline { 2 - 3 } & $\mathrm{M} s$ & Time period index. \\
\cline { 2 - 3 } & $\mathrm{P} s j m$ & Pumber of machines in stage $s$. \\
\cline { 2 - 3 } & $\mathrm{SUP} i j s$ & Setup time from job $i$ to job $j$ at stage $s$. \\
\cline { 2 - 3 } & $\mathrm{B} s$ & Buffer capacity at Stage $s$. \\
\cline { 2 - 3 } & $\mathrm{M}$ & Large Number. \\
\cline { 2 - 3 } & $\mathrm{T}$ & Makespan of arbitrary solution. \\
\cline { 2 - 3 } & $\mathrm{S}$ & Total number of stages. \\
\hline \multirow{4}{*}{\begin{tabular}{l} 
Variables \\
\cline { 2 - 3 }
\end{tabular}} & $\mathrm{C} s j m$ & Departure time of job $j$ from machine $m$ in stage $s$. \\
\cline { 2 - 3 } & $\mathrm{K} s j$ & Departure time of job $j$ from stage $s$. \\
\cline { 2 - 3 } & $\mathrm{F} s j$ & The processing and blocking time of job $j$ in stage $s$. \\
\cline { 2 - 3 } & $\mathrm{E}$ & Makespan \\
\hline
\end{tabular}




\begin{tabular}{|c|c|c|}
\hline Variable & Notation & Definition \\
\hline \multirow{5}{*}{$\begin{array}{l}\text { Binary } \\
\text { Decision } \\
\text { Variables }\end{array}$} & Xsjm & $\begin{array}{l}=1 \text { if job } j \text { is processed on machine } m \text { in stage } s, \\
=0 \text { otherwise. }\end{array}$ \\
\hline & Wsijm & $\begin{array}{l}=1 \text { if job } j \text { directly follows job } i \text { on machine } m \text { at stage } s, \\
=0 \text { otherwise. }\end{array}$ \\
\hline & $\mathrm{D} 1 s j t$ & $\begin{array}{l}=1 \text { if time period } t \text { precedes the starting time of job } j \text { at } \\
\text { stage } s \text {, } \\
=0 \text { otherwise. }\end{array}$ \\
\hline & D2sjt & $\begin{array}{l}=1 \text { if time period } t \text { is after the departure time of job } j \text { at } \\
\text { stage } s-1 \text {, } \\
=0 \text { otherwise. }\end{array}$ \\
\hline & Dsjt & $\begin{array}{l}=1 \text { if job } j \text { is in the buffer preceding stage } s \text { at time } t \text {, } \\
=0 \text { otherwise. }\end{array}$ \\
\hline
\end{tabular}

\section{Objective Function}

Minimize makespan, E.

\section{Constraints:}

There is a zero job that is used to define the setup time for the first job to be processed on each machine. The zero job has no value for processing time, but the setup times from zero to other jobs vary.

The first constraint ensure that the completion time of job $j$ is greater than or equal to the processing and setup time for job $j$ in the first stage. The second constraint guarantees that if job $j$ is processed on machine $m$ in stage $s$, the completion time of job $j$ is greater than or equal to the completion time of the preceding job plus the processing and setup time for job $j$. Constraint two also assures that the jobs are not interrupted during processing. The third constraint ensures that if job $j$ is the first job processed on machine $m$ in stage $s$ then the completion time is greater than or equal to the processing and setup time for job $j$ plus the completion time for job $j$ in stage $s-1$. 


$$
\begin{aligned}
& C_{s j m} \geq P_{s j m} * X_{s j m}+W_{s i j m} * S U P_{i j s} ; \forall j, i \neq j, s=1, m=1 . . M s \\
& C_{s j m}-C_{s i m}+\mathrm{M} *\left(1-W_{s i j m}\right) \geq P_{s j m}+W_{s i j m} * S U P_{i j s} ; \forall s, j, i \neq j, m=1 . . M s \\
& C_{s j m}-K_{(s-1) j} \geq P_{s j m}-\mathrm{M} *\left(1-X_{s j m}\right)+W_{s i j m} * S U P_{i j s} ; \forall s \neq 1, j, i \neq j, m=1 . . M s
\end{aligned}
$$

The fourth and fifth constraints set the value for $K_{s j}$ as the time that job $j$ leaves stage $s$.

$$
\begin{aligned}
& C_{s j m} \leq K_{s j} ; \forall s, j, m=1 . . M s \\
& C_{s j m} \geq K_{s j}-\mathrm{M} *\left(1-X_{s j m}\right) ; \forall s, j, m=1 . . M s(5)
\end{aligned}
$$

Constraint six sets the makespan equal to the maximum finishing time of all jobs at the final stage.

$$
K_{(S) j} \leq E ; \forall j(6)
$$

Constraint seven ensures that each job is processed on one and only one machine in each stage.

$$
\sum_{m=1}^{M s} X_{s j m}=1 ; \forall s, j \neq 0(7)
$$

Constraint eight ensures that every job $j$ must follow another job $i$.

$$
X_{s j m}-\sum_{i<>j}^{J} W_{s i j m}=0 ; \forall s, j \neq 0, m=1 . . M s
$$

Constraint nine states that each job $i$ may or may not be followed by another job $j$, but it can only be followed by at most one job.

$$
X_{s i m}-\sum_{j<>i}^{J} W_{s i j m} \geq 0 ; \forall s, i, m=1 . . M s
$$


Constraint ten sets the processing and blocking time for job $j$ in stage $s$. It is the difference of the finishing time of job $j$ and the preceding job $i$ in stage $s . F_{s j}$ is used in calculating the contents of the buffers.

$$
F_{s j} \leq K_{s j}-K_{s i}+M^{*}\left(1-W_{s i j m}\right) ; \forall s, j, i \neq j, m=1 . . M s
$$

Constraints eleven, twelve, and thirteen are the buffer constraints. $D 1_{s j t}$ is set to equal 1 if time period $t$ is before the start of processing job $j$ in stage $s . D 2_{s j t}$ is set to equal 1 if job $j$ has left stage $(s-1)$. Constraint fourteen sets $D_{s j t}$ equal to 1 only when $D 1_{s j t}$ and $D 2_{s j t}$ are both equal to 1 . If $D_{s j t}$ is equal to 1 then job $j$ is in the buffer before stage $s$ at time $t$, because job $j$ has left stage $(s-1)$ but it has not started processing on stage $s$. From the time a job enters the system until it leaves it is being setup, processed, blocked, or in a buffer.

$$
\begin{aligned}
& K_{s j}-F_{s j}-t-M^{*} D 1_{s j t}+1 \leq 0 ; \forall s, j, t \\
& t-K_{(s-1) j}-M^{*} D 2_{s j t} \leq 0 ; \forall s \neq 1, j, t \\
& D_{s j t}=D 1_{s j t}+D 2_{s j t}-1 ; \quad \forall s \neq 1, j, t
\end{aligned}
$$

Constraint fourteen sets the limits on the buffer's capacity. For each stage there is a maximum limit on the number of jobs that will fit into the buffer $B s$.

$$
\sum_{j}^{J} D_{s j t} \leq B s ; \forall s \neq 1, t
$$


Constraints fifteen and sixteen ensure that job 0 is processed first on every machine. This is needed to calculate the setup times for the first job processed on each machine at each stage.

$$
\begin{aligned}
& X_{s 0 m}=1 ; \quad \forall s, m=1 . . M s \\
& \sum_{i \diamond j}^{J} W_{s i 0 m}=0 ; \quad \forall s, j, m=1 . . M s
\end{aligned}
$$




\section{$5 \quad$ Heuristics}

Minimizing the makespan in a flexible flowshop with sequence dependent setup times, uniform machines, and limited buffers is considered to be strongly NP-Hard; exact methods are only practical for extremely small problems. To solve problems of this complexity, heuristic algorithms are needed. Heuristics are algorithms designed to give a good solution to a problem in a reasonable amount of time. The solution given by a heuristic may not be optimal, but it should be relatively close to optimal. Heuristics sacrifice some solution quality, but make up for it by being superior in computation time. For example, to optimally solve a three stage problem with three machines in the first stage, one machine in the second stage, one machine in the third stage, and 5 jobs took around one hour, but by using the heuristics given in this research, a five stage, thirty job problem can be solved in less than three minutes.

Two construction heuristics are developed in this research. The solutions from each construction heuristic are used in a simulated annealing meta-heuristic, that is designed for solution improvement. The two construction heuristics, RBFFS and PBFFS, use the same solution representation, but the jobs are assigned to machines in different ways. RBFFS is route based while PBFFS is job priority based. The simulated annealing meta-heuristic uses the same solution representation as the construction heuristics, and the same job/machine assignments corresponding to the construction heuristics. The solutions are represented by a priority permutation of the jobs to be scheduled in the system. This representation limits the solution space to $n$ ! solutions, where $n$ is the number of jobs. For example if there are five jobs to be scheduled in the system, a solution could be represented as $\{2,3,1,5,4\}$. 
The construction heuristics are based on the NEH algorithm developed by Nawaz, Enscore, and Ham (1983). The NEH algorithm was developed for the multistage flowshop environment. It gives higher priority to jobs with larger total processing times. A priority list is made by arranging the jobs in decreasing order of their total processing time on all machines. Next, the two jobs with the highest priority are chosen and the makespans for job 1 followed by job 2 and for job 2 followed by job 1 are calculated. The sequence with the smallest makespan is chosen as the best sequence, either 1-2 or 21 , then the job with the third highest priority enters the system. When job 3 enters the system, adjacent pairwise exchange is used to create three new partial sequences, and the makespan for each partial sequence is calculated. The partial sequence with the smallest makespan is chosen as the best three job sequence. This process continues until all jobs are scheduled. This heuristic looks at only $\{[n(n+1) / 2]-1\}$ permutation solutions as opposed to all $n$ ! solutions that exist, where $n$ equals the number of jobs. Many researchers reference this algorithm as one of the best for constructing a solution for the flowshop scheduling problem. A modified version of the NEH algorithm is used in this research to construct a solution for the flexible flowshop with sequence dependent setup times, uniform machines, and limited buffers.

\subsection{RBFFS Construction Heuristic}

The RBFFS construction heuristic uses a priority routing system that assigns jobs specific routes through the system. A route is a path that the job will travel through the system. A job's route dictates the machine it will visit at each stage, and the job that it will follow on that machine. The routes are designed so that the machines in each stage 
will operate for, as much as possible, the same amount of time. For the purpose of assigning the routes, all jobs are assumed to have the average base processing time. A ratio is then used with the machine speeds to decide on how many jobs will visit each machine. For example, if there are two machines at a stage, the first having a speed of 1.00 and the second a speed of 0.50 , the ratio would equal: $[(1.00 / 0.50)=2]$. By using this ratio, 2 jobs would be processed on machine one for every 1 job processed on machine two. In this heuristic, the order in which jobs will be processed on each machine is predetermined; it is based on the routing system, the machine configuration, and the job priority. Each job is given a route and follows that route through the system. In this heuristic, a machine may be idle sometimes while it is waiting for a specific job, even though other jobs may be ready for processing. In some cases, inserted idle time can be beneficial to the system.

The steps for the RBFFS construction algorithm are as follows:

1. Sum the processing times on the fastest machine at each stage for each job.

2. Arrange the jobs in descending order of the sums of their processing times. The jobs with the greatest sum of processing times are given the highest scheduling priority.

3. Determine the routes that will be used by the jobs in order to navigate through the system; details of route assignments are shown in the following example. The makespan is calculated by using the job processing times and the routes associated with each job. When the jobs are placed on different routes the makespan may increase or decrease. 
4. Take the two jobs with the highest priority and schedule them, using pairwise adjacent exchange. Calculate the makespan for each configuration and set the best RBFFS sequence as the sequence with the smallest makespan.

5. Choose the job with the next highest priority and enter it at the end of the best RBFFS sequence. Use pairwise adjacent exchange and calculate the makespan for each partial sequence. Set the best RBFFS sequence as the partial sequence with the smallest makespan.

6. If all jobs have been scheduled go to step 7, else go to step 5 .

7. Set the best $\mathrm{n}$ job sequence as the RBFFS sequence and the best makespan as the RBFFS makespan. STOP.

\subsection{RBFFS Construction Heuristic Example}

An example illustrating the construction heuristic is given in this section. It is an instance with 4 jobs that need to be scheduled in a 3 stage environment with 3 machines at the first stage, 1 machine at the second stage, and 2 machines at the third. The buffers are limited to three jobs. The first machine at any stage is the fastest and considered to have a speed of 1.00 , any other machine has a speed of 0.50 . This means that the fastest machine is twice as fast as the other machines. The processing and setup times are given below.

Table 5-1 Construction Heuristic Ex. Process Times Stage 1

\begin{tabular}{|l|c|c|c|c|}
\hline \multicolumn{5}{|c|}{ Processing Times Stage 1 } \\
\hline & Job 1 & Job 2 & Job 3 & Job 4 \\
\hline Machine 1 & 4 & 14 & 16 & 10 \\
\hline Machine 2 & 8 & 28 & 32 & 20 \\
\hline Machine 3 & 8 & 28 & 32 & 20 \\
\hline
\end{tabular}


Table 5-2 Construction Heuristic Ex. Setup Times Stage 1

\begin{tabular}{|c|c|c|c|c|}
\hline \multicolumn{5}{|c|}{ Setup Times Stage 1 } \\
\hline & To 1 & To 2 & To 3 & To 4 \\
\hline From 0 & 1 & 4 & 5 & 3 \\
\hline From 1 & 0 & 3 & 3 & 4 \\
\hline From 2 & 1 & 0 & 6 & 3 \\
\hline From 3 & 1 & 3 & 0 & 3 \\
\hline From 4 & 1 & 3 & 3 & 0 \\
\hline
\end{tabular}

Table 5-3 Construction Heuristic Ex. Process Times Stage 2

\begin{tabular}{|c|c|c|c|c|}
\hline \multicolumn{5}{|c|}{ Processing Times Stage 2 } \\
\hline & Job 1 & Job 2 & Job 3 & Job 4 \\
\hline Machine 1 & 4 & 8 & 10 & 7 \\
\hline
\end{tabular}

Table 5-4 Construction Heuristic Ex. Setup Times Stage 2

\begin{tabular}{|c|c|c|c|c|}
\hline \multicolumn{5}{|c|}{ Setup Times Stage 2 } \\
\hline & To 1 & To 2 & To 3 & To 4 \\
\hline From 0 & 2 & 2 & 3 & 3 \\
\hline From 1 & 0 & 3 & 4 & 2 \\
\hline From 2 & 2 & 0 & 4 & 2 \\
\hline From 3 & 1 & 2 & 0 & 2 \\
\hline From 4 & 1 & 2 & 2 & 0 \\
\hline
\end{tabular}

Table 5-5 Construction Heuristic Ex. Process Times Stage 3

\begin{tabular}{|l|c|c|c|c|}
\hline \multicolumn{5}{|c|}{ Processing Times Stage 3 } \\
\hline & Job 1 & Job 2 & Job 3 & Job 4 \\
\hline Machine 1 & 6 & 9 & 14 & 9 \\
\hline Machine 2 & 12 & 18 & 28 & 18 \\
\hline
\end{tabular}


Table 5-6 Construction Heuristic Ex. Setup Times Stage 3

\begin{tabular}{|c|c|c|c|c|}
\hline \multicolumn{5}{|c|}{ Setup Times Stage 3 } \\
\hline & To 1 & To 2 & To 3 & To 4 \\
\hline From 0 & 2 & 2 & 4 & 2 \\
\hline From 1 & 0 & 4 & 3 & 4 \\
\hline From 2 & 1 & 0 & 4 & 2 \\
\hline From 3 & 1 & 2 & 0 & 2 \\
\hline From 4 & 1 & 2 & 3 & 0 \\
\hline
\end{tabular}

Step one, two, and three: sum the processing times on the fastest machine at each stage for each individual job. Then set the RBFFS priority list by placing the jobs in descending order of processing time sums.

Table 5-7 Step 1 of RBFFS Example.

\begin{tabular}{|c|c|c|c|c|}
\hline \multicolumn{5}{|c|}{$\begin{array}{l}\text { Step 1: Sum processing time for each job on the } \\
\text { fastest machine. }\end{array}$} \\
\hline & Stage 1 & Stage 2 & Stage 3 & $\sum \mathrm{pt}$ \\
\hline Job 1 & 4 & 4 & 6 & 14 \\
\hline Job 2 & 14 & 8 & 9 & 31 \\
\hline Job 3 & 16 & 10 & 14 & 40 \\
\hline Job 4 & 10 & 7 & 9 & 26 \\
\hline
\end{tabular}

Table 5-8 Step 2 of RBFFS Example.

\begin{tabular}{|c|c|}
\hline \multicolumn{2}{|c|}{ RBFFS Priority List } \\
\hline First & Job 3 \\
\hline Second & Job 2 \\
\hline Third & Job 4 \\
\hline Fourth & Job 1 \\
\hline
\end{tabular}

The proposed heuristic uses a priority routing system, Table 5.9 shows the priority routing for the example problem. By using the ratios in stage one, 2 jobs will be processed on machine one, for each job processed on machine two and three. Since there is only one machine at the second stage all of the jobs will be processed on it. At the third stage, 2 jobs will be processed on machine one for each job processed on machine two. 
Table 5-9 Priority Routing for RBFFS Example.

\begin{tabular}{|l|c|c|c|}
\hline & Stage 1 & Stage 2 & Stage3 \\
\hline Priority Route* 1 & $1-0$ & $1-0$ & $1-0$ \\
\hline Priority Route* 2 & $1-1$ & $1-1$ & $2-0$ \\
\hline Priority Route* 3 & $2-0$ & $1-2$ & $1-1$ \\
\hline Priority Route* 4 & $3-0$ & $1-3$ & $1-3$ \\
\hline
\end{tabular}

Key: The first digit is the machine that processes the job; the second digit is index of the job that it follows.

* It should be noted that the priority routing system for each machine configuration is unique, but they do have the common goal of machine utilization. The priority routing system is specifically designed for each problem instance.

Step four and five: start with the first two jobs on the RBFFS priority list, use pairwise adjacent exchange, calculate the makespans and choose the best. The first two jobs on the RBFFS priority list are job 3 and job 2, so the makespans for the combinations $\{3,2\}$ and $\{2,3\}$ will be calculated. Table 5.10 and 5.11 show the Gantt Charts for partial sequences $\{3,2\}$ and $\{2,3\}$ respectfully. 
Table 5-10 RBFFS Makespan for Sequence $\{3,2\}$

\begin{tabular}{|c|c|c|c|c|c|c|c|c|c|c|c|c|c|c|c|c|c|c|c|c|c|c|}
\hline & & 20 & 21 & 22 & 23 & 24 & 25 & 33 & 34 & 35 & 36 & 37 & 38 & 39 & 47 & 48 & 49 & 50 & 52 & 53 & 67 & 68 \\
\hline \multirow{3}{*}{ Stage 1} & $\mathrm{mc} 1$ & -0 & $\bar{E}$ & 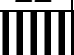 & |lा & ||ा| & $\|$ & |l||ा| & |ा| & IIII & $\prod$ & $\prod$ & IIIII & & & & & & & & & \\
\hline & $\mathrm{mc} 2$ & & & & & & & & & & & & & & & & & & & & & \\
\hline & $\mathrm{mc} 3$ & & & & & & & & & & & & & & & & & & & & & \\
\hline Stage 2 & $\mathrm{mc} 1$ & & & & & & & & $\overline{\bar{E}}$ & & & & & |||| & |||| & |||| & & & & & & \\
\hline \multirow{3}{*}{ Stage 3} & $\mathrm{mc} 1$ & & & & & & & & & $=$ & - & $\bar{~}$ & $\overline{\bar{L}}$ & $\overline{\bar{z}}$ & $=$ & & & & & & & \\
\hline & $\mathrm{mc} 2$ & & & & & & & & & & & & & & & & |III & $\|$ & |lा & |III & III & $\prod$ \\
\hline & & 20 & 21 & 22 & 23 & 24 & 25 & 33 & 34 & 35 & 36 & 37 & 38 & 39 & 47 & 48 & 49 & 50 & 52 & 53 & 67 & 68 \\
\hline \multicolumn{23}{|l|}{ Key } \\
\hline \multicolumn{23}{|l|}{ Job 1} \\
\hline \multicolumn{23}{|l|}{ Job 2} \\
\hline \multicolumn{23}{|l|}{ Job 3} \\
\hline Job 4 & & & & & & & & & & & & & & & & & & & & & & \\
\hline
\end{tabular}

Table 5-11 RBFFS Makespan for Sequence $\{2,3\}$

\begin{tabular}{|c|c|c|c|c|c|c|c|c|c|c|c|c|c|c|c|c|c|c|c|c|c|}
\hline & & 15 & 16 & 17 & 18 & 19 & 20 & 27 & 28 & 29 & 30 & 38 & 39 & 40 & 41 & 42 & 54 & 55 & 84 & 85 & 86 \\
\hline \multirow{3}{*}{ Stage 1} & $\mathrm{mc} 1$ & & & & & & & & & & & & & & & & & & & & \\
\hline & $\mathrm{mc} 2$ & & & & & & & & & & & & & & & & & & & & \\
\hline & $\mathrm{mc} 3$ & & & & & & & & & & & & & & & & & & & & \\
\hline Stage 2 & $\mathrm{mc} 1$ & & & & & & & & & & & & & & & & & & & & \\
\hline \multirow{3}{*}{ Stage 3} & $\mathrm{mc} 1$ & & & & & & & & & II & & & & & & & & & & & \\
\hline & $\mathrm{mc} 2$ & & & & & & & & & & & & & & & & & $\bar{E}$ & $\equiv$ & $\equiv$ & $\equiv$ \\
\hline & & 15 & 16 & 17 & 18 & 19 & 20 & 27 & 28 & 29 & 30 & 38 & 39 & 40 & 41 & 42 & 54 & 55 & 84 & 85 & 86 \\
\hline
\end{tabular}


Since this is such a small example the limited buffers do not cause any machine blockage. The partial solution $\{3,2\}$ is chosen with a makespan of 68 . The next job to inter is job 4 , so the partial solutions that will be considered are: $\{3,2,4\},\{3,4,2\}$, and $\{4,3,2\}$.

Tables 5.12, 5.13, and 5.14, show the Gantt charts representing partial sequences $\{3,2,4\},\{3,4,2\}$, and $\{4,3,2\}$ respectfully.

Table 5-12 RBFFS Makespan for Sequence $\{3,2,4\}$

\begin{tabular}{|c|c|c|c|c|c|c|c|c|c|c|c|c|c|c|c|c|c|c|c|c|c|c|c|c|c|c|}
\hline & & 20 & 21 & 22 & 23 & 24 & 33 & 34 & 35 & 36 & 37 & 38 & 39 & 40 & 47 & 48 & 49 & 50 & 51 & 52 & 53 & 56 & 57 & 58 & 67 & 68 \\
\hline \multirow{3}{*}{ Stage 1} & $\mathrm{mc} 1$ & & & \|\|$\|$ & IIII & \|\|$\|$ & IIII & \|\|\|\| & IIII & IIII & $\|[\|\|$ & \|\|$\|$ & & & & & & & & & & & & & & \\
\hline & $\mathrm{mc} 2$ & & & & & & & & & & & & & & & & & & & & & & & & & \\
\hline & $\mathrm{mc} 3$ & & & & & & & & & & & & & & & & & & & & & & & & & \\
\hline Stage 2 & mc 1 & & & $\overline{\bar{z}}$ & $\overline{\bar{~}}$ & 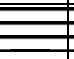 & $\overline{\bar{E}}$ & 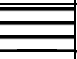 & & & & & \|\|$\|$ & |l|| & \|\| & \|\|$\|$ & & & & & & & & & & \\
\hline \multirow{3}{*}{ Stage 3} & $\mathrm{mc} 1$ & & & & & & & & & & & & & & & & & & & & & & & & & \\
\hline & mc 2 & & & & & & & & & & & & & & & & \|\| & ||$[\mid \|$ & ||l| & \|\| & \|\|$\|$ & \|\||| & $\|$ & \|\| & $\|[\|$ & $\||\|| \mid$ \\
\hline & & 20 & 21 & 22 & 23 & 24 & 33 & 34 & 35 & 36 & 37 & 38 & 39 & 40 & 47 & 48 & 49 & 50 & 51 & 52 & 53 & 56 & 57 & 58 & 67 & 68 \\
\hline \multicolumn{27}{|l|}{ Key } \\
\hline \multicolumn{27}{|l|}{ Job 1} \\
\hline \multicolumn{27}{|l|}{ Job 2} \\
\hline \multicolumn{27}{|l|}{ Job 3} \\
\hline Job 4 & & & & & & & & & & & & & & & & & & & & & & & & & & \\
\hline
\end{tabular}

Table 5-13 RBFFS Makespan for Sequence $\{3,4,2\}$

\begin{tabular}{|c|c|c|c|c|c|c|c|c|c|c|c|c|c|c|c|c|c|c|c|c|c|c|}
\hline & & 20 & 21 & 22 & 23 & 31 & 32 & 33 & 34 & 35 & 36 & 42 & 43 & 44 & 45 & 52 & 53 & 54 & 55 & 62 & 63 & 64 \\
\hline \multirow{3}{*}{ Stage 1} & $\mathrm{mc} 1$ & & & & & & & & & & & & & & & & & & & & & \\
\hline & $\mathrm{mc} 2$ & & & 10 & $m$ & 11 & 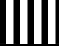 & & & & & & & & & & & & & & & \\
\hline & $\mathrm{mc} 3$ & & & & & & & & & & & & & & & & & & & & & \\
\hline Stage 2 & $\mathrm{mc} 1$ & & & & & & & & $\overline{\underline{\underline{\underline{E}}}}$ & & & & & II & & $\|$ & & & & & & \\
\hline \multirow{3}{*}{ Stage 3} & $\mathrm{mc} 1$ & & & & & & & & & $\overline{\underline{\underline{E}}}$ & $\overline{\bar{E}}$ & $\bar{E}$ & $\bar{E}$ & 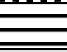 & $\bar{E}$ & $\bar{E}$ & & II & & II & & \\
\hline & $\mathrm{mc} 2$ & & & & & & & & & & & & & & & & & & & & & \\
\hline & & 20 & 21 & 22 & 23 & 31 & 32 & 33 & 34 & 35 & 36 & 42 & 43 & 44 & 45 & 52 & 53 & 54 & 55 & 62 & 63 & 64 \\
\hline
\end{tabular}


Table 5-14 RBFFS Makespan for Sequence $\{4,3,2\}$

\begin{tabular}{|c|c|c|c|c|c|c|c|c|c|c|c|c|c|c|c|c|c|c|c|c|c|c|c|c|c|c|}
\hline & & 12 & 13 & 14 & 15 & 22 & 23 & 24 & 25 & 31 & 32 & 33 & 34 & 35 & 43 & 44 & 45 & 46 & 53 & 54 & 55 & 64 & 65 & 66 & 67 & 76 \\
\hline \multirow{3}{*}{ Stage 1} & $\mathrm{mc} 1$ & & & & & & & & & & & & & & & & & & & & & & & & & \\
\hline & $\mathrm{mc} 2$ & & & & & & & & & & & & & & & & & & & & & & & & & \\
\hline & $\mathrm{mc} 3$ & & & & & & & & & & & & & & & & & & & & & & & & & \\
\hline Stage 2 & $\mathrm{mc} 1$ & & & & & & & & & & & & & & & & Ш & & & & & & & & & \\
\hline \multirow{3}{*}{ Stage 3} & $\mathrm{mc} 1$ & & & & & & & & & & & & & & & & & & & & & & & & & \\
\hline & $\mathrm{mc} 2$ & & & & & & & & & & & & & & & & & & & & & & & & & \\
\hline & & 12 & 13 & 14 & 15 & 22 & 23 & 24 & 25 & 31 & 32 & 33 & 34 & 35 & 43 & 44 & 45 & 46 & 53 & 54 & 55 & 64 & 65 & 66 & 67 & 76 \\
\hline \multicolumn{27}{|l|}{ Key } \\
\hline \multicolumn{27}{|l|}{ Job 1} \\
\hline \multicolumn{27}{|l|}{ Job 2} \\
\hline \multicolumn{27}{|l|}{ Job 3} \\
\hline Job 4 & & & & & & & & & & & & & & & & & & & & & & & & & & \\
\hline
\end{tabular}

The partial solution $\{3,4,2\}$ is chosen with a makespan of 64 . The next job to inter is job 1 , so the partial solutions that will be considered are: $\{3,4,2,1\},\{3,4,1,2\},\{3,1,4,2\}$, and $\{1,3,4,2\}$. Tables 5.15, 5.16, 5.17, and 5.18 show the Gantt charts representing partial sequences $\{3,4,2,1\},\{3,4,1,2\},\{3,1,4,2\}$, and $\{1,3,4,2\}$ respectfully.

Table 5-15 RBFFS Makespan for Sequence $\{3,4,2,1\}$

\begin{tabular}{|c|c|c|c|c|c|c|c|c|c|c|c|c|c|c|c|c|c|c|c|c|c|c|c|c|c|c|c|}
\hline & & 8 & 9 & 10 & 20 & 21 & 22 & 23 & 31 & 32 & 33 & 34 & 35 & 42 & 43 & 44 & 45 & 51 & 52 & 53 & 54 & 58 & 59 & 60 & 64 & 65 & 71 \\
\hline \multirow{3}{*}{ Stage 1} & $\mathrm{mc} 1$ & & & & & & & & & & & & & & & & & & & & & & & & & & \\
\hline & $\mathrm{mc} 2$ & & 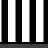 & & & & & & & & & & & & & & & & & & & & & & & & \\
\hline & $\mathrm{mc} 3$ & & & & & & & & & & & & & & & & & & & & & & & & & & \\
\hline Stage 2 & $\mathrm{mc} 1$ & & & & & & & & & & & $=$ & & & & 1 & & & & & & & & & & & \\
\hline \multirow{3}{*}{ Stage 3} & $\mathrm{mc} 1$ & & & & & & & & & & & & & & & & & & & & & & & & & & \\
\hline & $\mathrm{mc} 2$ & & & & & & & & & & & & & & & & & & & & & & & & & & \\
\hline & & 8 & 9 & 10 & 20 & 21 & 22 & 23 & 31 & 32 & 33 & 34 & 35 & 42 & 43 & 44 & 45 & 51 & 52 & 53 & 54 & 58 & 59 & 60 & 64 & 65 & 71 \\
\hline
\end{tabular}


Table 5-16 RBFFS Makespan for Sequence $\{3,4,1,2\}$

\begin{tabular}{|c|c|c|c|c|c|c|c|c|c|c|c|c|c|c|c|c|c|c|c|c|c|c|c|c|}
\hline & & 8 & 9 & 10 & 21 & 22 & 32 & 33 & 34 & 35 & 43 & 44 & 47 & 48 & 49 & 51 & 52 & 53 & 58 & 59 & 60 & 63 & 71 & 72 \\
\hline \multirow{3}{*}{ Stage 1} & mc 1 & & & & & & & & & & & & & & & & & & & & & & & \\
\hline & $\mathrm{mc} 2$ & & & & & & & & & & & & & & & & & & & & & & & \\
\hline & $\mathrm{mc} 3$ & II & & & & & & & & & & & & & & & & & & & & & & \\
\hline Stage 2 & mc 1 & & & & & & & & $=$ & & & & & & & & & & & & & & & \\
\hline \multirow{3}{*}{ Stage 3} & mc 1 & & & & & & & & & & & & & & & & & & & & & & & \\
\hline & $\mathrm{mc} 2$ & & & & & & & & & & & & & & & & & & & & & & & \\
\hline & & 8 & 9 & 10 & 21 & 22 & 32 & 33 & 34 & 35 & 43 & 44 & 47 & 48 & 49 & 51 & 52 & 53 & 58 & 59 & 60 & 63 & 71 & 72 \\
\hline Key & & & & & & & & & & & & & & & & & & & & & & & & \\
\hline Job 1 & & & & & & & & & & & & & & & & & & & & & & & & \\
\hline Job 2 & & & & & & & & & & & & & & & & & & & & & & & & \\
\hline Job 3 & & & & & & & & & & & & & & & & & & & & & & & & \\
\hline Job 4 & & & & & & & & & & & & & & & & & & & & & & & & \\
\hline
\end{tabular}

Table 5-17 RBFFS Makespan for Sequence $\{3,1,4,2\}$

\begin{tabular}{|c|c|c|c|c|c|c|c|c|c|c|c|c|c|c|c|c|c|c|c|c|c|c|c|c|}
\hline & & 20 & 21 & 22 & 23 & 24 & 26 & 27 & 32 & 33 & 34 & 38 & 39 & 40 & 48 & 49 & 52 & 53 & 57 & 58 & 59 & 63 & 73 & 74 \\
\hline \multirow{3}{*}{ Stage 1} & $\mathrm{mc} 1$ & & & & & & & & & & & & & & & & & & & & & & & \\
\hline & $\mathrm{mc} 2$ & & & & & & & & & & & & & & & & & & & & & & & \\
\hline & $\mathrm{mc} 3$ & & & & & & & & & & & & & & & & & & & & & & & \\
\hline Stage 2 & $\mathrm{mc} 1$ & & & & & & & & & & & & & & & & & & & & & & & \\
\hline \multirow{3}{*}{ Stage 3} & $\mathrm{mc} 1$ & & & & & & & & & & & & & & & & & . & & & & & & \\
\hline & $\mathrm{mc} 2$ & & & & & & & & & & & & & & & & & & & & & & & \\
\hline & & 20 & 21 & 22 & 23 & 24 & 26 & 27 & 32 & 33 & 34 & 38 & 39 & 40 & 48 & 49 & 52 & 53 & 57 & 58 & 59 & 63 & 73 & 74 \\
\hline
\end{tabular}


Table 5-18 RBFFS Makespan for Sequence $\{1,3,4,2\}$

\begin{tabular}{|c|c|c|c|c|c|c|c|c|c|c|c|c|c|c|c|c|c|c|c|c|c|c|c|c|c|}
\hline & & 4 & 5 & 11 & 12 & 18 & 19 & 20 & 24 & 25 & 31 & 32 & 33 & 38 & 39 & 47 & 48 & 56 & 57 & 58 & 59 & 60 & 61 & 70 & 71 \\
\hline \multirow{3}{*}{ Stage 1} & mc 1 & & & & & & & & $\overline{\bar{C}}$ & & & & & & & & & & & & & & & & \\
\hline & $\mathrm{mc} 2$ & & & & & & & & & & & & & & & & & & & & & & & & \\
\hline & $\mathrm{mc} 3$ & & & & & & & & & & & & & & & & & & & & & & & & \\
\hline Stage 2 & mc 1 & & & & & & & & & & & & & $\overline{\bar{z}}$ & & & & & & & & & & & \\
\hline \multirow{3}{*}{ Stage 3} & mc 1 & & & & & & & & & & & & & & & & & & & & & & & & \\
\hline & $\mathrm{mc} 2$ & & & & & & & & & & & & & & & & & & & & & & & & \\
\hline & & 4 & 5 & 11 & 12 & 18 & 19 & 20 & 24 & 25 & 31 & 32 & 33 & 38 & 39 & 47 & 48 & 56 & 57 & 58 & 59 & 60 & 61 & 70 & 71 \\
\hline
\end{tabular}

The final RBFFS solution is chosen as $\{3,4,2,1\}$ or $\{1,3,4,2\}$, since there is a tie, either solution can be chosen. Arbitrarily solution $\{3,4,2,1\}$ is chosen, so job 3 is given priority route* 1 and job 4 is given priority route* 2 , etc. The final makespan, or objective function is represented simply as 71 .

Step 7: the RBFFS sequence is $\{3,4,2,1\}$ and the corresponding makespan is 71 units. STOP

The final RBFFS sequence is then used as the starting solution in a simulated annealing algorithm. 


\subsection{RBFFS Simulated Annealing Heuristic}

Simulated annealing is a technique used to find good solutions to combinatorial problems; the solutions may or may not be optimal. It originated from the physical annealing of solids, and the connection between annealing and mathematical problems was first made by Metropolis et al. (1953). Later, Kirkpatirick et al. (1983) used it to solve combinatorial optimization problems, and it has shown good results. It looks at random variations of the current solution. Sometimes a non-improving solution is accepted as the new solution with a probability that decreases as the algorithm progresses. By sometimes accepting a non-improving solution, the simulated annealing algorithm avoids being trapped in local optima and can search different areas in the solution space for the global optima. Many researchers have had good results in using simulated annealing to solve flowshop scheduling problems.

The simulated annealing algorithm takes the final sequence from the RBFFS construction algorithm and searches for improved solutions by rearranging the sequence. This SA algorithm uses the same solution representation as the RBFFS heuristic, so the solution space is also limited to $n$ ! solutions while in reality there may be many more possible solutions. The solutions are listed as permutations of jobs, and use the priority route* based scheduling as in the RBFFS construction heuristic. The nomenclature used in the simulated annealing heuristic is given in Table 5.19. 
Table 5-19 Simulated Annealing Nomenclature

\begin{tabular}{|c|l|}
\hline Variable & Definition \\
\hline$n$ & Total number of jobs. \\
\hline$m$ & Total number of stages. \\
\hline$T$ & Temperature \\
\hline$N$ & Number of iterations at the current temperature. \\
\hline minT & Final temperature value. \\
\hline maxN & Max number of iterations at each temperature setting. \\
\hline RBFFSms & Makespan obtained from the construction heuristic. \\
\hline RBFFSseq & Sequence of jobs obtained from the construction heuristic. \\
\hline bestms & Lowest makespan \\
\hline bestseq & Sequence of jobs corresponding to the lowest makespan. \\
\hline curbestms & Current best makespan. \\
\hline curbestseq & Sequence of jobs corresponding to the current best makespan \\
\hline$Y$ & Random integer value between 1 and $\mathrm{n}$, including 1 and $\mathrm{n}$. \\
\hline$Z$ & Random integer value between 1 and $\mathrm{n}$, including 1 and $\mathrm{n}$. \\
\hline$R$ & Random value between 0 and 1. \\
\hline SAms & Simulated Annealing makespan. \\
\hline SAseq & Simulated Annealing sequence. \\
\hline$\Delta T C$ & Value of (currbestms - SAms) \\
\hline$\alpha$ & Temperature reduction factor. \\
\hline
\end{tabular}

The solution is represented in the SA heuristic the same way as in the construction heuristic, as a sequence of jobs such as $\{3,4,2,1\}$ where the first job is given priority route* 1 , in this case job 3 . The performance of each solution is based on its makespan, which is the completion time of the last job completed at stage $m$.

The steps for the SA algorithm are as follows.

1. Set the heuristic parameters $\min T, \max N, \alpha, T$, and $N$. The final temperature value $\min T$ dictates the length of the SA heuristic, i.e. the heuristic will run until $T$ reaches $\min T$. The maximum number of iterations at each temperature setting $\max N$, is one of the factors in determining how fast the temperature reduces. It regulates how many iterations are ran at a given probability of accepting a nonimproving solution. The temperature reduction factor $\alpha$, ranges between 0 and 1 , 
and is the other factor in determining how fast the temperature is reduced. The initial temperature $T$ is a factor in the acceptance of a non-improving solution. The higher the temperature, the greater the probability of accepting a nonimproving solution, which allows the heuristic to search different areas of the solution space. The initial value of $N$, the number of iterations at each temperature setting is set equal to 0 .

2. Set bestms $=$ RBFFSms, and bestseq $=$ RBFFSseq. In this step the best solution and sequence are set to the solution and sequence obtained from the RBFFS construction algorithm. Also the current makespan and sequence are set equal to the construction heuristic's makespan and sequence, curbestms $=$ RBFFSms and curbestseq $=$ RBFFSseq .

3. Generate random values for $Y$ and $Z$. The jobs in these two places are the jobs chosen for pairwise interchange; e.g. if $Y=10$ and $Z=6$, then the job assigned to priority route* 10 will be assigned to priority route* 6 and the job originally assigned priority route* 6 will now be assigned to priority route* $10 . Y$ and $Z$ represent locations in the priority list, and not actual job numbers.

4. If $Y$ is equal to $Z$ then go back to step 3; else go to step 5

5. Calculate the makespan after the exchange and set it equal to $S A m s$ also set the new sequence as SAseq.

6. Calculate $\triangle T C$ (curbestms $-S A m s)$. In this step, the difference between the current best makespan and the simulated annealing makespan is calculated.

7. If $\Delta T C>0.001$ then set curbestms $=S A m s$ and curbestseq $=S A s e q$. Step 7 checks the difference between the curbest makespan and the simulated annealing 
makespan, if it is greater than 0.001 then it means that the SA solution is better than the curbest. If this is the case then the curbest values are reset as the SA values. Also if $\quad \Delta T C>0.001$ then the simulated annealing solution is compared to the overall best solution found so far. If $S A m s<$ bestms then set bestms $=$ SAms and bestseq $=$ SAseq.

8. If $\Delta T C<0.001$ and $e^{\Delta T C / T}>\mathrm{R}$, then set curbestms $=$ SAms and curbestseq $=$ SAseq. If the SA solution is non-improving, step 8 checks to see if it will be accepted anyway. $R$ is a random value between 0.00 and 1.00. As the increase in the makespan gets smaller, the non-improving solution has a higher chance of being accepted. Also as $T$ gets smaller the probability of accepting a nonimproving solution decreases.

9. Set $N=N+1$.

10. If $N=\max N$ then $T=\alpha * T$ and $N=0$. This step checks how many iterations have taken place at the current temperature. If the maximum number of iterations at this temperature has been reached, $\max N$, then the value of $T$ is reset to the current temperature multiplied by the temperature reduction factor. After the new temperature is calculated it is compared with the minimum temperature, if $T<=$ $\min T$, the heuristic terminates, else go back to step 3.

The process flow chart for the simulated annealing heuristic is shown in Figure 5.1. 


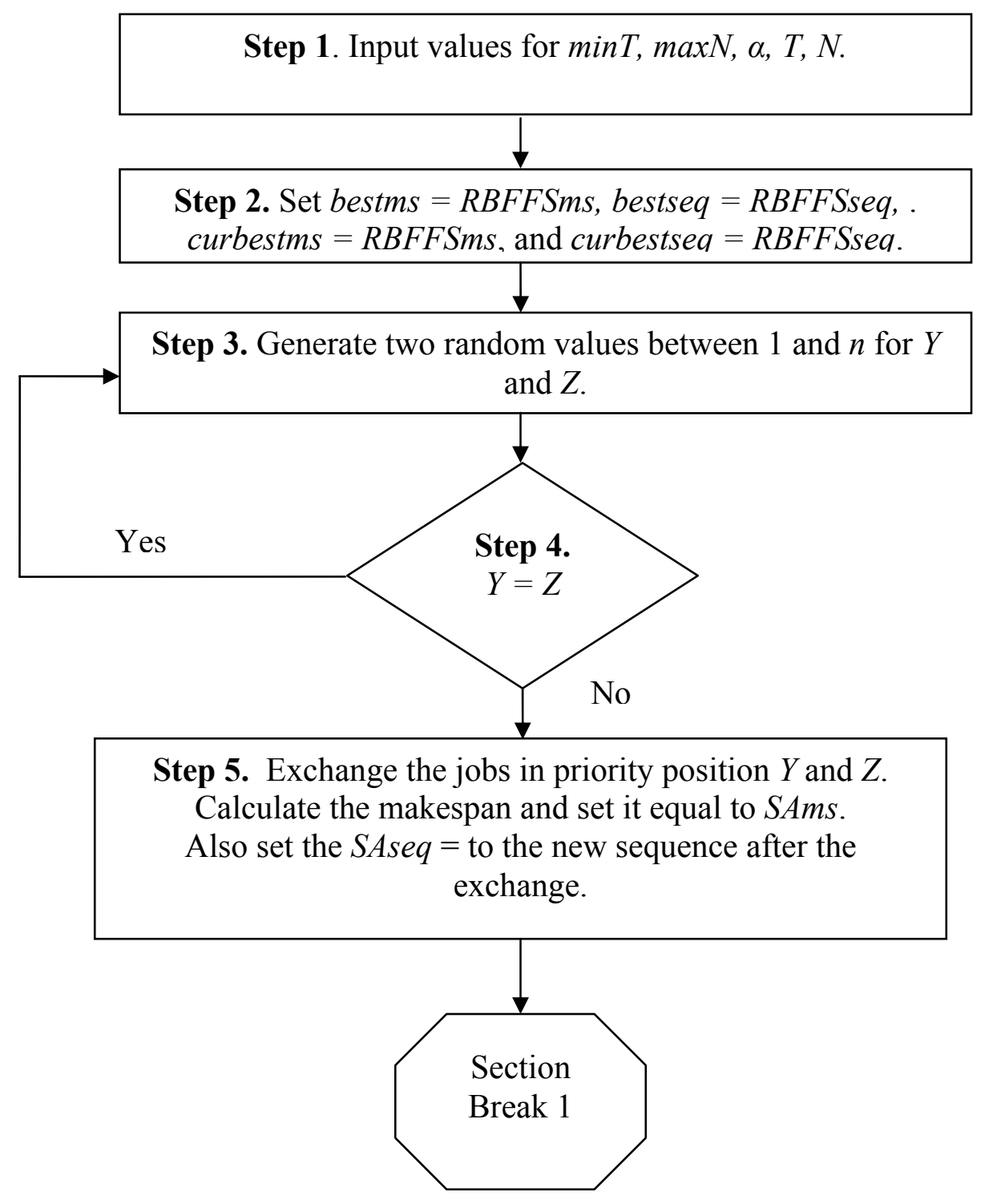

Figure 5.1 Simulated Annealing Process Flow Diagram 


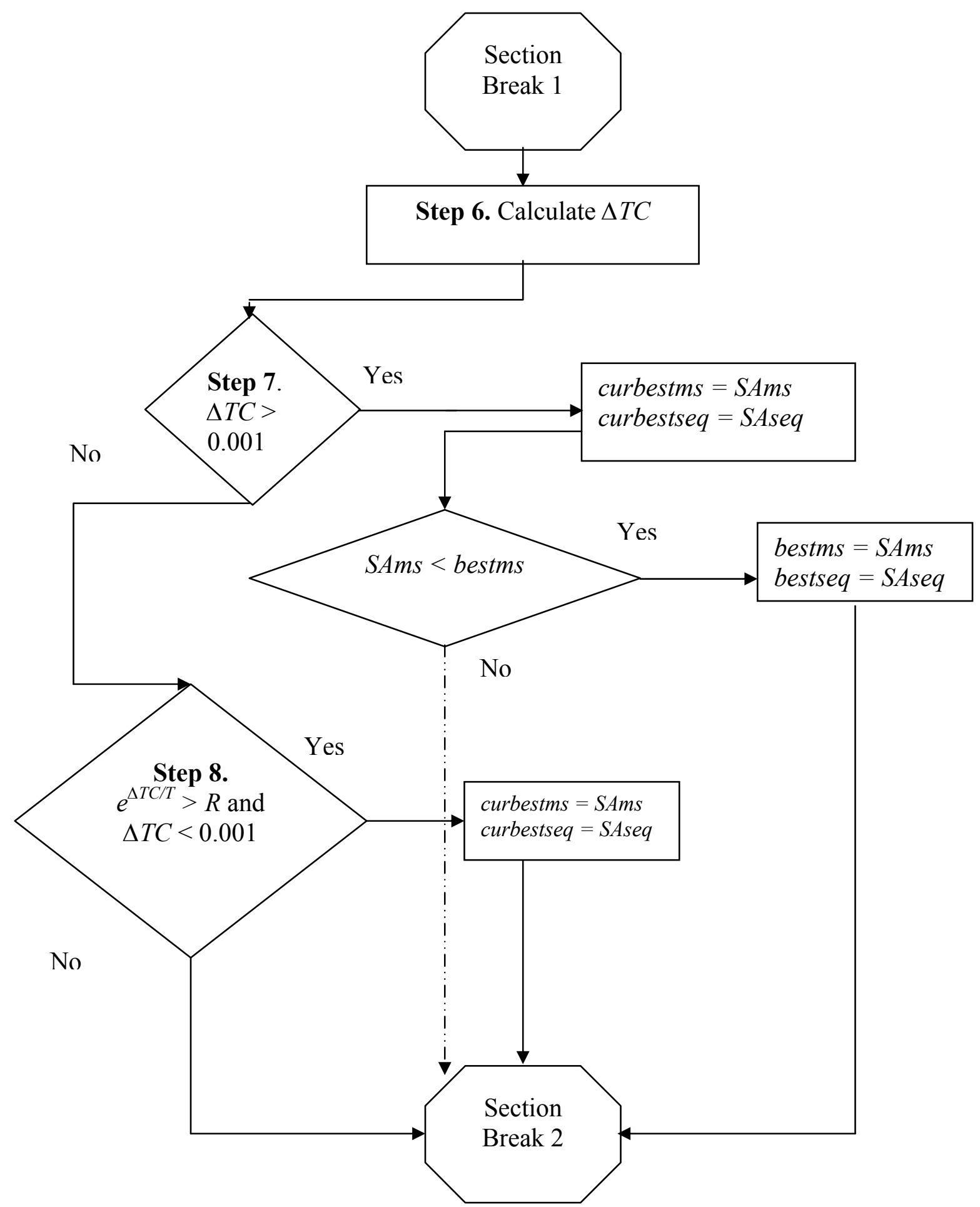

Figure 5.1 cont: Simulated Annealing Process Flow Diagram 


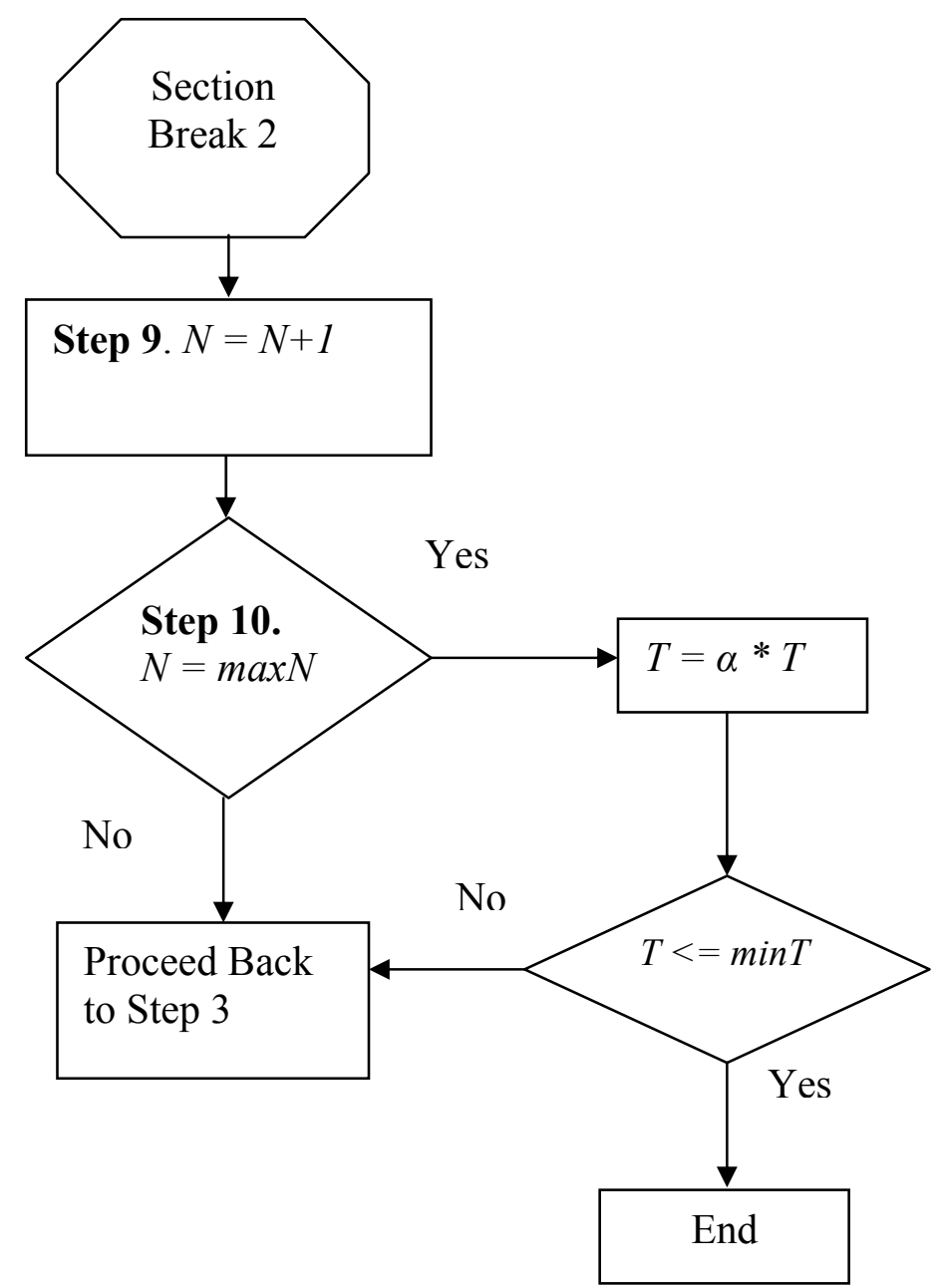

Figure 5.1 cont: Simulated Annealing Process Flow Diagram 


\subsection{Route Based Simulated Annealing Example}

A simple illustration of the simulated annealing heuristic is now given. The illustration will use the same data as the RBFFS construction heuristic. The starting solution for the simulated annealing heuristic is the final solution of the RBFFS construction heuristic, so the starting solution for SA is $\{3,4,2,1\}$.

Step 1: $\min T=80, \max N=1, \alpha=0.80, T=100, N=0$

Step 2: bestms $=71$, bestseq $=\{3,4,2,1\}$, curbestms $=71$, curbestseq $=\{3,4,2,1\}$

Step 3: $Y=2, Z=4$.

Step 4: $Y \neq Z$

Step 5: SAseq $=\{3,1,2,4\}$, SAms $=74$

Step 6: $\Delta T C=($ curbestms $-S A m s)=(71-74)=-3$

Step 7: $\triangle T C$ is not greater than 0.001 .

Step 8: $e^{\Delta T C / T}=e^{-3 / 100}=0.97, R=0.25$, Also $\Delta T C<0.001$, so the non-improving solution

is accepted. curbestms $=74$, curbestseq $=\{3,1,2,4\}$.

Step 9: $N=0+1=1$.

Step 10: $N=\max N$, so $T=100 * 0.80=80$. Since $T<=\min T$ the heuristic is stopped.

\subsection{PBFFS Construction Heuristic}

The PBFFS construction heuristic is a job priority based heuristic. Each job is given a priority index, and when a machine becomes open the available job with the lowest index is assigned to that machine. The priority given to each job is the same for all stages; it is an entire system priority. The solution is represented as a list of jobs with 
the first job listed as having a priority of 1 . For example a four job problem may have the solution $\{3,4,1,2\}$, where job 3 has the highest priority, job 4 has the next highest, job 1 the next, and job 2 has the lowest priority. As with the other heuristic, this solution technique limits the solution space to $n$ ! solutions, where $n$ is the number of jobs. The PBFFS heuristic has the same procedure as RBFFS except, the job assignments are not route based. In the PBFFS, jobs are scheduled based on two criteria, their priority index, and their availability for processing. The job order for each machine in this case is not known in advance; it depends on the job's priority and processing times.

The steps for the PBFFS heuristic are as follows:

1. Sum the processing times on the fastest machine at each stage for each job.

2. Arrange the jobs in descending order of the sums of their processing times. The jobs with the greatest sum of processing times are given the highest scheduling priority. Assign each job a priority, a number from 1 to $n$, where 1 is the highest priority.

3. Set all machine open times equal to 0 . All jobs are initially ready for processing at stage 1, but will not become available for stage 2 until after they are finished processing at stage 1 . This holds true for all stages 3,4 , etc.

4. Take the two jobs with the highest priority and schedule them, using pairwise adjacent exchange. Start scheduling jobs at stage one starting with the first machine; assign the available job with the highest priority. As jobs are scheduled and processed, the machine open time is calculated 
as (the previous job completion time + job setup time + job processing time + any blocking time that may occur). Jobs are scheduled on the machine with the lowest open time, until all jobs have been processed through the system. If there is a tie on machine open time, the job will be processed on the machine with the smallest machine index, ex. mc $1, \mathrm{mc}$

2, or mc 3. Calculate the makespan for each configuration and set the best PBFFS sequence as the sequence with the smallest makespan.

5. Choose the job with the next highest priority and enter it at the end of the best PBFFS sequence. Use pairwise adjacent exchange and calculated the makespan for each partial sequence. Set the best PBFFS sequence as the partial sequence with the smallest makespan.

6. If all jobs have been scheduled, go to step 7. Else go to step 5.

7. Set the best $n$ job sequence as the PBFFS sequence and the best makespan as the PBFFS makespan. STOP.

\subsection{PBFFS Construction Heuristic Example}

An illustration of the PBFFS construction heuristic is shown using the same job data that were used in the RBFFS example. The steps for PBFFS are the same as RBFFS except the jobs are scheduled in a different way. Since the example is small the limited buffers do not block any machines.

Step one two, and three: sum the processing times on the fastest machine at each stage, for each individual job. Then set the PBFFS priority list by place the jobs in descending order and assigning a priority index to each job. 
Table 5-20 Step 1 of PBFFS Example.

\begin{tabular}{|c|c|c|c|c|}
\hline \multicolumn{5}{|c|}{$\begin{array}{l}\text { Step 1: Sum processing time for each job on the } \\
\text { fastest machine. }\end{array}$} \\
\hline & Stage 1 & Stage 2 & Stage 3 & $\Sigma \mathrm{pt}$ \\
\hline Job 1 & 4 & 4 & 6 & 14 \\
\hline Job 2 & 14 & 8 & 9 & 31 \\
\hline Job 3 & 16 & 10 & 14 & 40 \\
\hline Job 4 & 10 & 7 & 9 & 26 \\
\hline
\end{tabular}

Table 5-21 Step 2 and 3 of PBFFS Example.

\begin{tabular}{|c|c|}
\hline \multicolumn{2}{|c|}{ PBFFS Priority List } \\
\hline First & Job 3 \\
\hline Second & Job 2 \\
\hline Third & Job 4 \\
\hline Fourth & Job 1 \\
\hline
\end{tabular}

Step four and five: start with the first two jobs on the PBFFS priority list, use pairwise adjacent exchange, calculate the makespans and choose the best. The first two jobs on the PBFFS priority list are job 3 and job 2, so the makespans for the combinations $\{3,2\}$ and $\{2,3\}$ will be calculated. Table 5.22 and 5.23 show the Gantt Charts for partial priority lists $\{3,2\}$ and $\{2,3\}$ respectfully. 
Table 5-22 PBFFS Makespan for Priority $\{3,2\}$

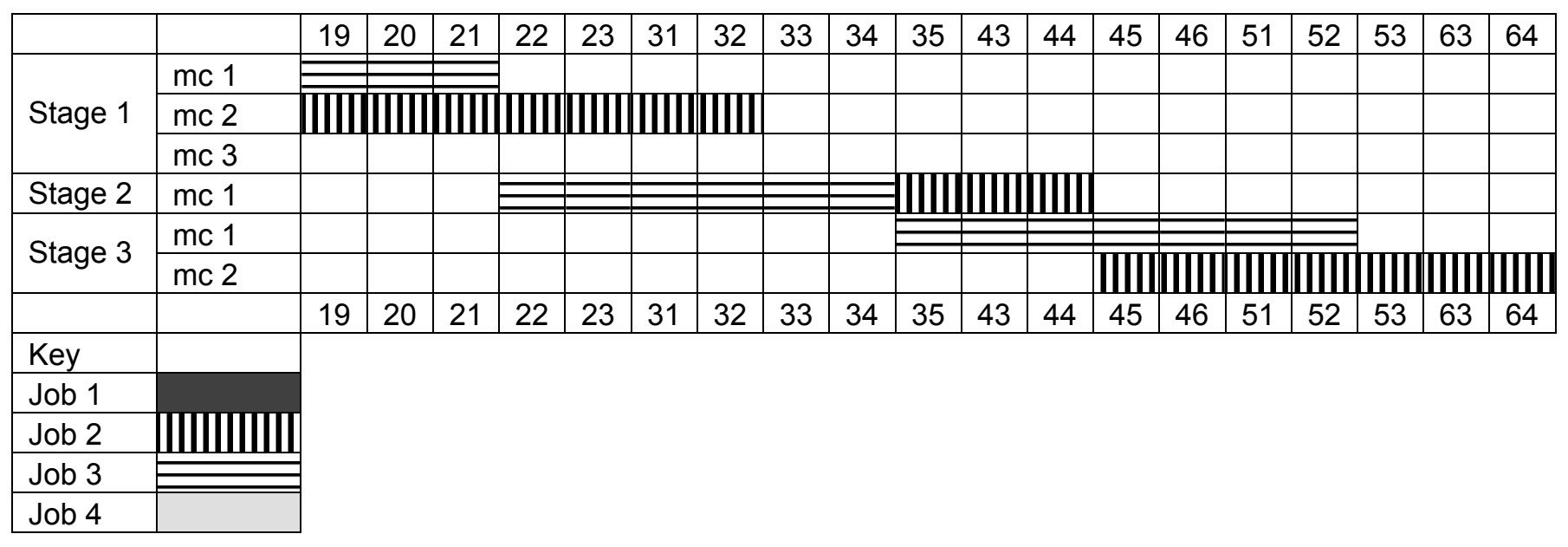

Table 5-23 PBFFS Makespan for Priority $\{2,3\}$

\begin{tabular}{|c|c|c|c|c|c|c|c|c|c|c|c|c|c|c|c|c|c|c|}
\hline & & 17 & 18 & 19 & 20 & 27 & 28 & 29 & 36 & 37 & 38 & 39 & 40 & 50 & 51 & 52 & 82 & 83 \\
\hline \multirow{3}{*}{ Stage 1} & mc 1 & & & & & & & & & & & & & & & & & \\
\hline & $\mathrm{mc} 2$ & & & & & & & & & $\bar{~}$ & & & & & & & & \\
\hline & $\mathrm{mc} 3$ & & & & & & & & & & & & & & & & & \\
\hline Stage 2 & mc 1 & & & & & & & & & & & & & & & & & \\
\hline \multirow{3}{*}{ Stage 3} & mc 1 & & & & & & & & & & & & & & & & & \\
\hline & $\mathrm{mc} 2$ & & & & & & & & & & & & & & & & & \\
\hline & & 17 & 18 & 19 & 20 & 27 & 28 & 29 & 36 & 37 & 38 & 39 & 40 & 50 & 51 & 52 & 82 & 83 \\
\hline
\end{tabular}


The partial solution $\{3,2\}$ is chosen with a makespan of 64 . The next job to inter is job 4 , so the partial solutions that will be considered are: $\{3,2,4\},\{3,4,2\}$, and $\{4,3,2\}$. Tables 5.24, 5.25, and 5.26, show the Gantt charts representing partial sequences $\{3,2,4\},\{3,4,2\}$, and $\{4,3,2\}$ respectfully.

Table 5-24 PBFFS Makespan for Priority $\{3,2,4\}$

\begin{tabular}{|c|c|c|c|c|c|c|c|c|c|c|c|c|c|c|c|c|c|c|}
\hline & & 20 & 21 & 22 & 23 & 24 & 32 & 33 & 34 & 35 & 43 & 44 & 45 & 51 & 52 & 53 & 54 & 64 \\
\hline \multirow{3}{*}{ Stage 1} & $\mathrm{mc} 1$ & & & & & & & & & & & & & & & & & \\
\hline & $\mathrm{mc} 2$ & $\|\mid\|$ & \|\| & $\|||$ & || & $\|$ & $|\|| \mid$ & & & & & & & & & & & \\
\hline & $\mathrm{mc} 3$ & & & & & & & & & & & & & & & & & \\
\hline Stage 2 & $\mathrm{mc} 1$ & & & & & & & & 5 & $|\|| \mid$ & $\|[\|$ & $|\|| \mid$ & & & & & & \\
\hline \multirow{3}{*}{ Stage 3} & $\mathrm{mc} 1$ & & & & & & & & & $=$ & $=$ & & & & & & & \\
\hline & $\mathrm{mc} 2$ & & & & & & & & & & & & |l|||| & ||l|| & |IIII & |III| & 11 & $\|[\|$ \\
\hline & & 20 & 21 & 22 & 23 & 24 & 32 & 33 & 34 & 35 & 43 & 44 & 45 & 51 & 52 & 53 & 54 & 64 \\
\hline
\end{tabular}

Table 5-25 PBFFS Makespan for Priority $\{3,4,2\}$

\begin{tabular}{|c|c|c|c|c|c|c|c|c|c|c|c|c|c|c|c|c|c|c|c|c|c|}
\hline & & 20 & 21 & 22 & 23 & 24 & 31 & 32 & 33 & 34 & 35 & 42 & 43 & 44 & 51 & 52 & 53 & 54 & 62 & 63 & 64 \\
\hline \multirow{3}{*}{ Stage 1} & $\mathrm{mc} 1$ & & & & & & & & & & & & & & & & & & & & \\
\hline & $\mathrm{mc} 2$ & & & & & & & & & & & & & & & & & & & & \\
\hline & $\mathrm{mc} 3$ & |l|| & ||| & |||| & 川I & & & II & & & & & & & & & & & & & \\
\hline Stage 2 & $\mathrm{mc} 1$ & & & & & & & & & & & & & & III & & & & & & \\
\hline \multirow{3}{*}{ Stage 3} & $\mathrm{mc} 1$ & & & & & & & & & & & & & & & & & 11 & II & III & II \\
\hline & $\mathrm{mc} 2$ & & & & & & & & & & & & & & & & & & & & \\
\hline & & 20 & 21 & 22 & 23 & 24 & 31 & 32 & 33 & 34 & 35 & 42 & 43 & 44 & 51 & 52 & 53 & 54 & 62 & 63 & 64 \\
\hline
\end{tabular}


Table 5-26 PBFFS Makespan for Priority $\{4,3,2\}$

\begin{tabular}{|c|c|c|c|c|c|c|c|c|c|c|c|c|c|c|c|c|c|c|c|c|c|}
\hline & & 12 & 13 & 14 & 23 & 24 & 32 & 33 & 34 & 35 & 37 & 38 & 42 & 43 & 44 & 56 & 57 & 61 & 62 & 63 & 73 \\
\hline \multirow{3}{*}{ Stage 1} & $\mathrm{mc} 1$ & & & & & & & & & & & & & & & & & & & & \\
\hline & $\mathrm{mc} 2$ & & & & & & & & 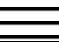 & 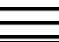 & $=$ & & & & & & & & & & \\
\hline & $\mathrm{mc} 3$ & & & & & & & & & & & & & & & & & & & & \\
\hline Stage 2 & $\mathrm{mc} 1$ & & & & & & & & & & & & & & & & & & & & \\
\hline \multirow{3}{*}{ Stage 3} & $\mathrm{mc} 1$ & & & & & & & & & & & & & & & & & & & & \\
\hline & $\mathrm{mc} 2$ & & & & & & & & & & & & & & & & & & & & \\
\hline & & 12 & 13 & 14 & 23 & 24 & 32 & 33 & 34 & 35 & 37 & 38 & 42 & 43 & 44 & 56 & 57 & 61 & 62 & 63 & 73 \\
\hline
\end{tabular}

The partial solution $\{3,2,4\}$ and $\{3,4,2\}$ each have a makespan of 64 , so either partial sequence can be used. Arbitrarily the partial sequence $\{3,4,2\}$, is chosen. The next job to enter is job 1 , so the partial solutions that will be considered are: $\{3,4,2,1\},\{3,4,1$, $2\},\{3,1,4,2\}$, and $\{1,3,4,2\}$. Tables 5.27, 5.28, 5.29, and 5.30 show the Gantt charts representing partial sequences $\{3,4,2,1\}$, $\{3,4,1,2\},\{3,1,4,2\}$, and $\{1,3,4,2\}$ respectfully. 
Table 5-27 PBFFS Makespan for Priority $\{3,4,2,1\}$

\begin{tabular}{|c|c|c|c|c|c|c|c|c|c|c|c|c|c|c|c|c|c|c|c|c|c|c|c|}
\hline & & 21 & 22 & 23 & 24 & 26 & 27 & 32 & 33 & 34 & 35 & 43 & 44 & 51 & 52 & 53 & 54 & 59 & 60 & 63 & 64 & 65 & 76 \\
\hline \multirow{3}{*}{ Stage 1} & $\mathrm{mc} 1$ & & & & & & & & & & & & & & & & & & & & & & \\
\hline & $\mathrm{mc} 2$ & & & & & & & & & & & & & & & & & & & & & & \\
\hline & $\mathrm{mc} 3$ & & & & & & & & & & & & & & & & & & & & & & \\
\hline Stage 2 & $\mathrm{mc} 1$ & & & & & & & & & & & & & & & & & & & & & & \\
\hline \multirow{3}{*}{ Stage 3} & $\mathrm{mc} 1$ & & & & & & & & & & & & & & $\bar{~}$ & & & & & & & & \\
\hline & $\mathrm{mc} 2$ & & & & & & & & & & & & & & & & & & & & & & \\
\hline & & 21 & 22 & 23 & 24 & 26 & 27 & 32 & 33 & 34 & 35 & 43 & 44 & 51 & 52 & 53 & 54 & 59 & 60 & 63 & 64 & 65 & 76 \\
\hline
\end{tabular}

Table 5-28 PBFFS Makespan for Priority $\{3,4,1,2\}$

\begin{tabular}{|c|c|c|c|c|c|c|c|c|c|c|c|c|c|c|c|c|c|c|c|c|c|c|c|}
\hline & & 8 & 9 & 10 & 15 & 16 & 21 & 22 & 23 & 24 & 34 & 35 & 40 & 41 & 43 & 44 & 53 & 54 & 55 & 56 & 57 & 66 & 67 \\
\hline \multirow{3}{*}{ Stage 1} & mc 1 & & & & & & & & & & & & & & & & & & & & & & \\
\hline & $\mathrm{mc} 2$ & & & & & & & & & & & & & & & & & & & & & & \\
\hline & $\mathrm{mc} 3$ & & & $\|$ & & & & & & & & & & & & & & & & & & & \\
\hline Stage 2 & $\mathrm{mc} 1$ & & & & & & & & & & & & & & & & & & & & & & \\
\hline \multirow{3}{*}{ Stage 3} & $\mathrm{mc} 1$ & & & & & & & & & & & & & & & & & & & & & & \\
\hline & $\mathrm{mc} 2$ & & & & & & & & & & & & & & & & & & & & & & \\
\hline & & 8 & 9 & 10 & 15 & 16 & 21 & 22 & 23 & 24 & 34 & 35 & 40 & 41 & 43 & 44 & 53 & 54 & 55 & 56 & 57 & 66 & 67 \\
\hline
\end{tabular}

Table 5-29 PBFFS Makespan for Priority $\{3,1,4,2\}$

\begin{tabular}{|c|c|c|c|c|c|c|c|c|c|c|c|c|c|c|c|c|c|c|c|c|c|c|c|}
\hline & & 8 & 9 & 10 & 15 & 16 & 21 & 22 & 23 & 24 & 34 & 35 & 40 & 41 & 43 & 44 & 53 & 54 & 56 & 57 & 65 & 66 & 67 \\
\hline \multirow{3}{*}{ Stage 1} & mc 1 & & & & & & & & & & & & & & & & & & & & & & \\
\hline & mc 2 & & & & & & & & & & & & & & & & & & & & & & \\
\hline & $\mathrm{mc} 3$ & & & & & & & & & & & & & & & & & & & & & & \\
\hline Stage 2 & mc 1 & & & & & & & & & & $\bar{E}$ & & & & & & & & & & & & \\
\hline \multirow{3}{*}{ Stage 3} & mc 1 & & & & & & & & & & & & & & & & & & & & & & \\
\hline & $\mathrm{mc} 2$ & & & & & & & & & & & & & & & & & & & & & & \\
\hline & & 8 & 9 & 10 & 15 & 16 & 21 & 22 & 23 & 24 & 34 & 35 & 40 & 41 & 43 & 44 & 53 & 54 & 56 & 57 & 65 & 66 & 67 \\
\hline
\end{tabular}


Table 5-30 PBFFS Makespan for Priority $\{1,3,4,2\}$

\begin{tabular}{|c|c|c|c|c|c|c|c|c|c|c|c|c|c|c|c|c|c|c|c|c|c|c|c|c|c|}
\hline & & 4 & 5 & 6 & 11 & 12 & 19 & 22 & 23 & 24 & 31 & 32 & 33 & 36 & 37 & 42 & 43 & 52 & 53 & 54 & 55 & 56 & 57 & 73 & 74 \\
\hline \multirow{3}{*}{ Stage 1} & $\mathrm{mc} 1$ & & & 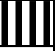 & II & $\prod$ & II & II & $\prod$ & & & & & & & & & & & & & & & & \\
\hline & $\mathrm{mc} 2$ & & & & & & & & & & & & & & $\bar{\nu}$ & & & & & & & & & & \\
\hline & $\mathrm{mc} 3$ & & & & & & & & & & & & & & & & & & & & & & & & \\
\hline Stage 2 & mc 1 & & & & & & & & & & & & W & Ш & IIII & & & & & & & & & & \\
\hline \multirow{3}{*}{ Stage 3} & $\mathrm{mc} 1$ & & & & & & & & & & & & & & & & III & IIII & III & 川 & \|\|$\|$ & & 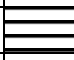 & & \\
\hline & $\mathrm{mc} 2$ & & & & & & & & & & & & & & & & & & & & & & & & \\
\hline & & 4 & 5 & 6 & 11 & 12 & 19 & 22 & 23 & 24 & 31 & 32 & 33 & 36 & 37 & 42 & 43 & 52 & 53 & 54 & 55 & 56 & 57 & 73 & 74 \\
\hline \multicolumn{26}{|l|}{ Key } \\
\hline \multicolumn{26}{|l|}{ Job 1} \\
\hline \multicolumn{26}{|l|}{ Job 2} \\
\hline \multicolumn{26}{|l|}{ Job 3} \\
\hline Job 4 & & & & & & & & & & & & & & & & & & & & & & & & & \\
\hline
\end{tabular}

The final PBFFS solution is chosen as $\{3,4,1,2\}$ or $\{3,1,4,2\}$, since there is a tie, either solution can be chosen. Arbitrarily solution $\{3,4,1,2\}$ is selected. The final makespan, or objective function is represented simply as 67 .

Step 7: the PBFFS sequence is $\{3,4,1,2\}$ and the corresponding makespan is 67 units. STOP

The final PBFFS sequence is then used as the starting solution in a simulated annealing algorithm. The simulated annealing algorithm is the same as the one used in conjunction with the RBFFS construction heuristic, and it adjusts the sequence in the same way; the only difference in the development of the schedules is when the makespans are calculated. The makespans are calculated using the method from the PBFFS construction heuristic. 


\section{$6 \quad$ Lower Bound}

The flexible flowshop with sequence dependent setup times, uniform machines, and limited buffers is a very complex problem, where only extremely small problems can be solved optimally. If the optimal solution cannot be found, other means for measuring the performance of the heuristics need to be employed. Three prominent ways of testing are using upper/lower bounds and comparing with other heuristics. In this research, lower bounds and comparison with other heuristics will be used. This section details how the lower bound is developed. The lower bound solution may be infeasible, but it gives a point of reference in testing the heuristic solutions. Due to the complexity of the problem, the lower bound presented is not very strong, and is not expected to be very close to the value of the optimal solution, but the complexity of the problem makes it very difficult to obtain a stronger lower bound. The lower bound presented was compared with LP relaxation for two randomly chosen medium sized problems, 4 stages and 30 jobs. The presented LB was $39.7 \%$ and $31.3 \%$ higher than the objective function of the LP relaxation solution.

Variable key for Lower Bound:

Table 6-1Lower Bound Nomenclature

\begin{tabular}{|l|l|}
\hline \multicolumn{1}{|c|}{ Variable } & \multicolumn{1}{c|}{ Description } \\
\hline$s$ & Stage index. \\
\hline$M s$ & The number of machines at each stage $s$. \\
\hline$B P T s$ & Sum of base processing times for stage $s$. \\
\hline$P P s$ & Processing power of stage $s$. \\
\hline$B s$ & $B P T s$ divided by $P P s$ for each stage $s$. \\
\hline$S S E T s$ & Sum of the smallest setup times for all jobs at each stage $s$. \\
\hline$D s$ & SSETs divided by $M s$ for each stage $s$. \\
\hline$G s$ & $\begin{array}{l}\text { The sum of } B s \text { and } D s \text { for each stage } s, \text { rounded up to the next } \\
\text { integer. }\end{array}$ \\
\hline$E s$ & $\begin{array}{l}\text { The largest sum (for any job) of the job basic processing time and } \\
\text { its smallest setup time for each stage } s .\end{array}$ \\
\hline
\end{tabular}




\begin{tabular}{|c|c|}
\hline Variable & Description \\
\hline$K s$ & The maximum between $G s$ and $E s$ for each stage $s$. \\
\hline$F$ & $\begin{array}{l}\text { The fastest time any job can reach the bottleneck stage, } \\
\text { including the setup times, from } 0 \text {. }\end{array}$ \\
\hline$F_{n}$ & The $\mathrm{n}^{\text {th }}$ fastest time a job can reach the bottleneck stage. \\
\hline Ls & $\begin{array}{l}\text { The fastest estimated completion times for two jobs in each } \\
\text { stage } s \text { leading up to the bottleneck stage. It is found by using } \\
\text { the } 2 \text { jobs with the minimum base processing times and set up } \\
\text { times at each stage. The jobs chosen at each of the stages are } \\
\text { independent. The value for } L s \text { is found by taking the } \\
\text { maximum value between (sum processing times / machine } \\
\text { processing power using the fastest } 2 \text { machines) + (smallest } \\
\text { setup times / number of machines at stage } s \text { (with a maximum } \\
\text { of } 2 \text { machines at stage } s \text { )), and (largest base processing time } \\
+ \text { smallest setup time for job with largest base processing } \\
\text { time)\} }\end{array}$ \\
\hline$H$ & $\begin{array}{l}\text { The sum of } L s \text {, for all stages leading up to the bottleneck } \\
\text { stage. ex. For stage } 3 \text { as the bottleneck, } H=L_{1}+L_{2}\end{array}$ \\
\hline SH & $\begin{array}{l}\text { The earliest possible time processing can start on the second } \\
\text { machine in the bottleneck stage. } S H=\max \left[H, F_{2}\right] \text {, where } F_{2} \\
\text { is the second smallest sum calculated in the determination of } \\
F \text {. }\end{array}$ \\
\hline$I$ & SH minus $F$. \\
\hline Os & $\begin{array}{l}\text { Similar to } L s \text { except that is uses the } 3 \text { smallest jobs at each } \\
\text { stage. }\end{array}$ \\
\hline$J$ & $\begin{array}{l}\text { The sum of } O s \text {, for all stages leading up to the bottleneck } \\
\text { stage. ex. For stage } 3 \text { as the bottleneck, } J=O_{1}+O_{2}\end{array}$ \\
\hline$S J$ & $\begin{array}{l}\text { The earliest possible time processing can start on the third } \\
\text { machine in the bottleneck stage. } S J=\max \left[J, F_{3}\right] \text {, where } F_{3} \text { is } \\
\text { the third smallest sum calculated in the determination of } F \text {. }\end{array}$ \\
\hline$Q$ & $S J$ minus $F$. \\
\hline$S 2 L B$ & Lower bound after step 2 . \\
\hline$B P T B N$ & Sum of the Base processing times at the bottleneck stage. \\
\hline$P M 2$ & Processing power of machine 2 at the bottleneck stage. \\
\hline$P M 3$ & Processing power of machine 3 at the bottleneck stage. \\
\hline$P B S$ & Processing power of all machines at the bottleneck stage. \\
\hline$P$ & $\begin{array}{l}\text { The smallest possible value for any job to finish processing } \\
\text { after the bottleneck stage including setup. }\end{array}$ \\
\hline$L B$ & Lower Bound \\
\hline
\end{tabular}

An example of the lower bound will be worked out with its presentation. The data used in the example are listed in Tables 6.2-6.6. For the example problem a four 
stage flexible flowshop with three machines in each of the stages is considered. There are four jobs to be processed, and for simplicity, all of the setup times in this illustration are considered to be equal to 1 . Also in the example, the first machine at each stage has a speed of $100 \%$ and all other machines have a speed of $50 \%$.

Table 6-2 Lower Bound Example Stage 1 Processing Times

\begin{tabular}{|l|c|c|c|c|c|}
\hline \multicolumn{7}{|c|}{ Stage 1 Processing Times } \\
\hline & Job 1 & Job 2 & Job 3 & Job 4 & $\sum \mathrm{pt}$ \\
\hline Machine 1 & 4 & 5 & 3 & 8 & 20 \\
\hline Machine 2 & 8 & 10 & 6 & 16 & 40 \\
\hline Machine 3 & 8 & 10 & 6 & 16 & 40 \\
\hline
\end{tabular}

Table 6-3 Lower Bound Example Setup Times

\begin{tabular}{|c|c|c|c|c|}
\hline \multicolumn{5}{|c|}{ All Stages Setup Times } \\
\hline & To Job 1 & Job 2 & Job 3 & Job 4 \\
\hline From Job 0 & 1 & 1 & 1 & 1 \\
\hline Job 1 & $\mathrm{X}$ & 1 & 1 & 1 \\
\hline Job 2 & 1 & $\mathrm{X}$ & 1 & 1 \\
\hline Job 3 & 1 & 1 & $\mathrm{X}$ & 1 \\
\hline Job 4 & 1 & 1 & 1 & $\mathrm{X}$ \\
\hline
\end{tabular}

Table 6-4Lower Bound Example Stage 2 Processing Times

\begin{tabular}{|c|c|c|c|c|c|}
\hline \multicolumn{7}{|c|}{ Stage 2 Processing Times } \\
\hline & Job 1 & Job 2 & Job 3 & Job 4 & $\sum \mathrm{pt}$ \\
\hline Machine 1 & 6 & 5 & 6 & 2 & 19 \\
\hline Machine 2 & 12 & 10 & 12 & 4 & 38 \\
\hline Machine 3 & 12 & 10 & 12 & 4 & 38 \\
\hline
\end{tabular}

Table 6-5 Lower Bound Example Stage 3 Processing Times

\begin{tabular}{|c|c|c|c|c|c|}
\hline \multicolumn{7}{|c|}{ Stage 3 Processing Times } \\
\hline & Job 1 & Job 2 & Job 3 & Job 4 & $\sum$ pt \\
\hline Machine 1 & 8 & 4 & 6 & 8 & 26 \\
\hline Machine 2 & 16 & 8 & 12 & 16 & 52 \\
\hline Machine 3 & 16 & 8 & 12 & 16 & 52 \\
\hline
\end{tabular}

Table 6-6 Lower Bound Example Stage 4 Processing Times

\begin{tabular}{|c|c|c|c|c|c|}
\hline \multicolumn{7}{|c|}{ Stage 4 Processing Times } \\
\hline & Job 1 & Job 2 & Job 3 & Job 4 & $\sum \mathrm{pt}$ \\
\hline Machine 1 & 2 & 6 & 5 & 2 & 15 \\
\hline Machine 2 & 4 & 12 & 10 & 4 & 30 \\
\hline Machine 3 & 4 & 12 & 10 & 4 & 30 \\
\hline
\end{tabular}




\subsection{Step 1 of the Lower Bound}

The lower bound is developed in three steps. In the first step, the bottleneck stage is determined. To identify the bottleneck stage, the sum of base processing times for each job is calculated for each stage and is designated as $(B P T s)$. Then $(B P T S)$ is divided by the processing power at that stage $(P P s)$, this becomes value $(B s)$. The processing power is basically the sum of the machine speeds. A more detailed description of processing power is given in the next paragraph. The smallest setup time is determined for each job, and the smallest setup times are added for all jobs and set equal to (SSETS). Next (SSETS), is divided by the total number of machines in the stage $(M s)$; this value is labeled as $(D s)$. The final value for this part of step one is found by adding $(B s)$ and $(D s)$ for each stage and rounding up to the next integer, this value becomes (Gs).

The processing power is calculated by summing the speeds of the machines. The processing times for jobs are calculated by dividing the base processing time by the speed of the machine. For example if a stage has three machines with speeds of $100 \%, 50 \%$, and $50 \%$, and a job has a base processing time of 6 units, the time to process that job on machine 1 will be $(6 / 1)=6$ units, machine 2 and $3(6 / 0.50)=12$ units. If a stage has only one machine then the processing power is 1 , for a stage with two machines the processing power is $(1.00+0.50)=1.50$, and for a stage with three machines the processing power is $(1.00+0.50+0.50)=2.00$. Also the largest sum of any single job's base processing time and its smallest setup time is calculated for each stage and labeled as (Es). For each stage, the fastest estimated completion time of all jobs is the largest value of either (Gs) or $(E s)$ and is labeled as $(K s)$. The first part of the lower bound determines the stage with the largest (Ks) which is the estimated completion time for that stage. The stage where 
the maximum $(K s)$ occurs is considered to be the bottleneck stage and the rest of the lower bound is based around this stage. It should be noted that the bottleneck stage is estimated using lower bounds and may not be the actual bottleneck stage. Using the data given in Tables 6.2- 6.6, calculations for step one of the lower bound are as follows:

For first stage, $K_{l}=\operatorname{Max}\{(20 / 2)+(4 / 3),(8+1)\}=\operatorname{Max}\{10+1.33,9\}=12$.

The second stage $K_{2}=\operatorname{Max}\{(19 / 2)+(4 / 3),(6+1)\}=\operatorname{Max}\{9.5+1.33,7\}=11$.

The third stage $K_{3}=\operatorname{Max}\{(26 / 2)+(4 / 3),(8+1)\}=\operatorname{Max}\{13+1.33,9\}=15$.

The fourth stage $K_{4}=\operatorname{Max}\{(15 / 2)+(4 / 3),(6+1)\}=\operatorname{Max}\{7.5+1.33,7\}=9$.

Since the third stage has the largest calculated value of $K_{s}$, it is considered to be the bottleneck.

\subsection{Step 2 of the Lower Bound}

The second step in the lower bound calculation accounts for the idle time in the bottleneck stage, before the processing of the first job. If the bottleneck stage is not the first stage then some idle time will occur on each of the machines at the bottleneck stage while they are waiting for jobs to arrive from the previous stages. To find the earliest possible start time for any machine at the bottleneck stage, the base processing and setup up times from zero for each job, at each stage preceding the bottleneck stage, are summed over all stages, and then the smallest total value is chosen and designated as value $(F)$. Table 6.7 shows how this is calculated for the example problem: 
Table 6-7 Step 2 Lower Bound

\begin{tabular}{|l|c|c|c|c|}
\hline & Job 1 & Job 2 & Job 3 & Job 4 \\
\hline Stage 1 & $4+1=5$ & $5+1=6$ & $3+1=4$ & $8+1=9$ \\
\hline Stage 2 & $6+1=7$ & $5+1=6$ & $6+1=7$ & $2+1=3$ \\
\hline Total & $\mathbf{1 2}$ & $\mathbf{1 2}$ & $\mathbf{1 1}$ & $\mathbf{1 2}$ \\
\hline
\end{tabular}

Job 3 would be chosen (with $\mathrm{F}=11$ units), so 11 idle units on all machines will be added to the lower bound. All machines at the considered bottleneck stage will be idle for at least 11 time units.

In addition to the idle time that all machines at the considered bottleneck stage will experience, additional idle time may occur on the second machine before it starts processing jobs. After the first machine at bottleneck stage begins processing, some additional idle time may occur on the other machines, because they may have to wait for the next available jobs to arrive. To account for the idle time on the second machine to begin processing at the bottleneck stage, the two jobs with the minimum values of the sum of basic processing times and the smallest setup times are chosen from each stage. The jobs chosen are independent for each stage, so if a job is chosen in one stage it does not have to be used at the following stages. After the jobs are chosen, an estimated completion time for each stage is obtained in a similar way as for value $(K s)$ in the first step of the lower bound. The estimated completion time for each stage $s$ is denoted as $(L s)$. The sum of the values for $(L s)$ leading up to the bottleneck stage is calculated and is denoted as $(H)$. The start time for the second machine at the bottleneck stage, denoted as $(\mathrm{SH}) . \mathrm{SH}$ is calculated as the maximum value between $\left[H, F_{2}\right]$. The extra idle time for 
the second machine at the bottleneck stage is calculated by subtracting $(F)$ from $(S H)$ and is denoted as value $(I)$, where (I) is greater than or equal to zero.

For the example, job 3 and job 1 are chosen from the first stage, job 4 and job 2 are chosen from the second stage.

The first stage $\left(L_{1}\right)$ value is $\operatorname{Max}\{(7 / 1.5)+(2 / 2),(4+1)\}=\operatorname{Max}\{4.67+1,5\}=6$.

The second stage $\left(L_{2}\right)$ value is $\operatorname{Max}\{(7 / 1.5)+(2 / 2),(5+1)\}=\operatorname{Max}\{4.67+1,6\}=6$. The value for $(H)$ is calculated as $6+6=12$.

The earliest time for a job to reach machine two at the bottleneck stage (SH) is calculated as $\operatorname{Max}\{12,12\}=12$. Since no jobs are ready for processing at the bottleneck stage until time period 11, and the earliest possible time a second job may be ready for processing is at time period 12 , one extra idle unit $(12-11=1)$ will be added to the second machine, $(I$ $=1)$.

The extra idle time for the third machine is calculated in the same way as for the second machine except the three jobs with the minimum values of the sum of the base processing time and smallest setup times are used. The estimated completion time for each stage $s$ is labeled as $(O s)$. The extra idle time for machine three is found by summing $(O s)$ for each stage leading up to the bottleneck stage and is designated as $(J)$. The start time for the third machine at the bottleneck stage, denoted as $(S J) . S J$ is calculated as the maximum $\left[J, F_{3}\right]$. The extra idle time units to add to the third machine are found by subtracting $(F)$ from $(S J)$ and this value is designated as $(Q)$, where $(Q)$ is greater than or equal to zero. 
For the example, jobs 3, 1, and 2 would be chosen from the first stage and job 4, 2 , and 1 or 3 would be chosen from the second stage. The jobs at each stage are treated independently.

The first stage value of $\mathrm{O}_{1}=\operatorname{Max}\{(12 / 2)+(3 / 3),(5+1)\}=\operatorname{Max}\{6+1,6\}=7$.

The second stage value of $\mathrm{O}_{2}=\operatorname{Max}\{(13 / 2)+(3 / 3),(6+1)\}=\operatorname{Max}\{6.5+1,7\}=8$.

The value of $\mathrm{J}$ is calculated as $7+8=15$.

The earliest time for a job to reach machine three in the bottleneck stage (SJ) is calculated as $\operatorname{Max}\{12,15\}$. Since the minimum time for the third job to reach the bottleneck stage is 15 time units, four extra idle units will be added to the starting time of the third machine at the bottleneck stage, $(Q)$ is calculated as $15-11=4$. This process is repeated until all lower bound extra idle times are determined for all of the machines at the bottleneck stage.

The last part of step 2 involves calculating the new lower bound after adding the idle times to the processing time for the bottleneck stage. This is done by using the following equation:

Step $2 \mathrm{LB}=\operatorname{Max}\left\{\left[\frac{B P T B N+(I \times P M 2)+(Q \times P M 3)}{P B S}+\mathrm{D}_{\mathrm{s}}\right], \mathrm{E}_{\mathrm{s}}\right\}+\mathrm{F}$

Step $2 \mathrm{LB} \quad=\quad$ Lower bound after step 2 .

$\mathrm{BPTBN}=\quad$ Sum of the base processing times at the bottleneck stage.

PM2 = Processing power of machine 2 at the bottleneck stage.

PM3 = Processing power of machine 3 at the bottleneck stage.

PBS $\quad=\quad$ Processing power of all machines at the bottleneck stage.

* It should be noted that the calculated value in the brackets [] is also rounded up. 
For the example the lower bound value after step 2 would be calculated as follows:

$$
\begin{aligned}
\text { Step 2 LB }= & \left.\left.\operatorname{Max} \frac{\{[26+(1 * 0.50)+(4 * 0.50)}{2}+(4 / 3)\right],(8+1)\right\}+11 \\
& =\quad \operatorname{Max}\{14.25+1.33,9\}+11 \\
& =\quad 27
\end{aligned}
$$

\subsection{Step 3 of the Lower Bound}

In the last step of the lower bound, extra time is added for the stages that follow the bottleneck stage. All jobs are considered. For each job, the base processing time for each of the post bottleneck stages is added to the smallest possible setup time, excluding the setup times from 0 . The smallest value between all the jobs is labeled as $(P) .(P)$ is then added to the lower bound that was calculated after step 2, and this value is the final lower bound, $(L B)$.

\section{$L B=\operatorname{Step} 2 \mathrm{LB}+P$}

For the example problem, the value for step three is calculated as follows:

Table 6-8 Step 3 Lower Bound

\begin{tabular}{|l|l|l|l|l|}
\hline & Job 1 & Job 2 & Job 3 & Job 4 \\
\hline Stage 4 & $2+1=3$ & $6+1=7$ & $5+1=6$ & $2+1=3$ \\
\hline
\end{tabular}

Job 1 or 4 is chosen to be the final job processed, and 3 time units are added to the last value obtained in step 2 . The final lower bound $(L B)$ is calculated as $27+3=30$. Even though the buffers in between stages have a limited capacity and blocking may occur; this could not be included in the calculations of the lower bound. 


\section{Simple Heuristics}

Two quick and easy simple heuristics are also developed as another means of testing the solution quality of the RBFFS and PBFFS heuristics. The first simple heuristic SH1 assigns jobs priority based on the order in which they are placed (FCFS). The second simple heuristic $\mathrm{SH} 2$ allocates priorities to jobs based on the largest total processing time (LTPT). The simple heuristics then process the jobs through the system and record the maximum completion time. In both heuristics the jobs that are ready for processing are assigned to the first available machine based on the job's priority. The job with priority 1 is chosen over all others, then the job with priority 2 , and so forth.

\subsection{Simple Heuristic One}

In the first simple heuristic, SH1, jobs are designated priorities on a first come

first serve basis, and then they are processed through the system. In this heuristic the jobs are assigned a priority index as they enter the system, for example job 1 would receive priority 1, and so forth. Table 7.1 shows an example of the first come FCFS heuristic, SH1. The example is calculated using the same data that were used in the construction heuristic example in section 5.2. The $\mathrm{SH} 1$ job priority index is $\{1,2,3,4\}$. 
Table 7-1 SH1 Example Gantt Chart

\begin{tabular}{|c|c|c|c|c|c|c|c|c|c|c|c|c|c|c|c|c|c|c|c|}
\hline & & 5 & 6 & 11 & 12 & 19 & 20 & \begin{tabular}{|l|}
28 \\
\end{tabular} & 29 & 32 & 33 & 37 & 42 & 43 & 48 & 49 & 55 & 56 & 74 \\
\hline \multirow{3}{*}{ Stage 1} & $\mathrm{mc} 1$ & & & & & & & & & & & & & & & & & & \\
\hline & $\mathrm{mc} 2$ & & & & & & & & & & & & & & & & & & \\
\hline & $\mathrm{mc} 3$ & & & & & & & & & & & 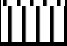 & & & & & & & \\
\hline Stage 2 & $\mathrm{mc} 1$ & & & & & & & & & & & & $\overline{\underline{ }}$ & 川 & 川 & & L & & \\
\hline \multirow{3}{*}{ Stage 3} & mc 1 & & & & & & & & & & & & & & & & & & \\
\hline & $\mathrm{mc} 2$ & & & & & & & & & & & & & & & & & & \\
\hline & & 5 & 6 & 11 & 12 & 19 & 20 & 28 & 29 & 32 & 33 & 37 & 42 & 43 & 48 & 49 & 55 & 56 & 74 \\
\hline \multicolumn{20}{|l|}{ Key } \\
\hline \multicolumn{20}{|l|}{ Job 1} \\
\hline \multicolumn{20}{|l|}{ Job 2} \\
\hline \multicolumn{20}{|l|}{ Job 3} \\
\hline Job 4 & & & & & & & & & & & & & & & & & & & \\
\hline
\end{tabular}

The corresponding makespan to the SH1 sequence $\{1,2,3,4\}$ is calculated as 74 .

\subsection{Simple Heuristic Two}

In the second simple heuristic $\mathrm{SH}$, the jobs are given priorities based on the largest processing time, with the largest one getting the highest priority. To calculate SH2, the LPT based simple heuristic, first the base processing times for each job at all stages must be added together. Table 7.2 shows the results of the summation of the base processing times.

Table 7-2 Sum of Base Processing Times

\begin{tabular}{|l|l|l|l|l|}
\hline & Job 1 & Job 2 & Job 3 & Job 4 \\
\hline Stage 1 & 4 & 14 & 16 & 10 \\
\hline Stage 2 & 4 & 8 & 10 & 7 \\
\hline Stage 3 & 6 & 9 & 14 & 9 \\
\hline Total & $\mathbf{1 4}$ & $\mathbf{3 1}$ & $\mathbf{4 0}$ & $\mathbf{2 6}$ \\
\hline
\end{tabular}

The priority list associated with this example would be $\{3,2,4,1\}$. The Gantt Chart for the SH2 makespan is shown in Table 7.3. 
Table 7-3 SH2 Example Gantt Chart

\begin{tabular}{|c|c|c|c|c|c|c|c|c|c|c|c|c|c|c|c|c|c|c|c|c|}
\hline & & 21 & 22 & 23 & 24 & 26 & 27 & 32 & 34 & 35 & 44 & 45 & 52 & 53 & 54 & 58 & 59 & 64 & 65 & 71 \\
\hline \multirow{3}{*}{ Stage 1} & $\mathrm{mc} 1$ & & & & & & & & & & & & & & & & & & & \\
\hline & $\mathrm{mc} 2$ & & & & $\|$ & & & & & & & & & & & & & & & \\
\hline & $\mathrm{mc} 3$ & & & & & & & & & & & & & & & & & & & \\
\hline Stage 2 & $\mathrm{mc} 1$ & & & & & & & & $\overline{\bar{C}}$ & 川 & $\|$ & & & & & & & & & \\
\hline \multirow{3}{*}{ Stage 3} & $\mathrm{mc} 1$ & & & & & & & & & & & & & & & & & & & \\
\hline & $\mathrm{mc} 2$ & & & & & & & & & & & & & & & & & & & \\
\hline & & 21 & 22 & 23 & 24 & 26 & 27 & 32 & 34 & 35 & 44 & 45 & 52 & 53 & 54 & 58 & 59 & 64 & 65 & 71 \\
\hline \multicolumn{21}{|l|}{ Key } \\
\hline \multicolumn{21}{|l|}{ Job 1} \\
\hline \multicolumn{21}{|l|}{ Job 2} \\
\hline \multicolumn{21}{|l|}{ Job 3} \\
\hline Job 4 & & & & & & & & & & & & & & & & & & & & \\
\hline
\end{tabular}

The corresponding makespan for the SH2 sequence $\{3,2,4,1\}$ is 71 units. Both of these simple heuristics provide a feasible solution that can be used as a reference, when determining the solution quality of the more complicated RBFFS and PBFFS heuristics. For the same example data, the construction heuristics RBFFS and PBFFS makespans were 71 and 67 respectively. 


\section{Experimentation}

Since this specific problem has not been addressed in the literature, a means of testing the proposed heuristics is needed. There are two methods that will be used to test the solution quality of each heuristic:

1. Comparison with a lower bound. By comparing with a lower bound, an idea is obtained on how good the solution is. Even though the lower bound may be infeasible and may be much lower than the optimal value, it is a good measuring tool for solution quality.

2. The second method used to test solution quality is to use other heuristics and compare the results. For comparison, two simple heuristics were developed. The first simple heuristic gives the jobs priority on a first come first serve basis. This would occur when jobs are sent to the end of the queue and wait for processing. The second simple heuristic gives jobs priority based on total processing time, where the jobs with larger total processing times get higher scheduling priority. While the lower bound may be infeasible, all heuristics developed in this research give feasible solutions.

\subsection{Problem Instances}

Three different simplified flexible flowshop environments were chosen to test the performance of the proposed heuristics. The first environment consists of 3 stages with a machine configuration of 3-1-2 and a limit of 3 jobs in the buffers between stages. This environment is modeled after part of a hydraulic hose manufacturing process. In the first 
stage the hoses are attached to a harness, in the second stage the hoses are put in an oven for heat treating, and in the last stage the fittings are assembled.

The second environment consists of 4 stages with a machine configuration of 2-1-2-1 and a limit of 3 jobs in the buffers between stages. This environment is modeled after a vinyl window manufacture. In the first stage the pieces of vinyl are cut to length, in the second stage the four sides of the window are welded together, in the third stage the latches and tracks are added, and in the last stage the final assembly takes place.

The third environment consists of 5 stages with a machine configuration of 3-3-2-2-1 and a limit of 4 jobs in buffers between stages. This environment is modeled after a high voltage circuit breaker assembly manufacturer. In the first stage painting is preformed, in the second stage wiring takes place, in the third mechanical assembly is done, in the fourth electrical assembly takes place, and in the last step the unit is tested.

\subsection{Data Generation}

Data were not available for the jobs in these manufacturing environments, so they were generated. The first machine at each stage is considered to be the fastest with a speed of 1.00 all other machines have a speed of 0.50 . The processing power of a stage is found by adding the speeds of all machines at that stage. The base processing time for a job at any stage is considered to be the job processing time on the fastest machine at that stage. The job setup time for any stage is between 20 to $40 \%$ of the job base processing time. The job processing times were generated as to not present any obvious bottleneck stages. If there were a bottleneck stage, the problem would basically reduce to a one stage scheduling problem. The data were generated as follows: 
- If a stage has 1 machine, then the base processing time is chosen form a uniform distribution between 2 and 10. This gives an average processing time of 6 units. The average processing time divided by the machine processing power is $(6 / 1)=6$ units.

- If a stage has 2 machines, then the base processing time is chosen from a uniform distribution between 3 and 15. This gives an average processing time of 9 units. The average processing time divided by the machine processing power $=(9 / 1.5)=6$ units.

- If a stage has 3 machines, then the base processing time is chosen from a uniform distribution between 4 and 20. This gives an average processing time of 12 units. The average processing time divided by the machine processing power $=(12 / 2)=6$ time units.

- Ten different data sets with 30 jobs each were generated for each of the three flowshop environments.

The nomenclature used in the experimentation is listed in Table 8.1.

Table 8-1 Experiment Nomenclature

\begin{tabular}{|c|l|}
\hline Nomenclature & Definition \\
\hline SH1 & $\begin{array}{l}\text { Refers to the simple heuristic, using first come first serve } \\
\text { priority allocation. }\end{array}$ \\
\hline SH2 & $\begin{array}{l}\text { Refers to the simple heuristic, using a largest total processing } \\
\text { time priority allocation. }\end{array}$ \\
\hline RBFFS & Refers to the route based construction heuristic. \\
\hline PBFFS & Refers to the priority based construction heuristic \\
\hline RBFFS_SA & $\begin{array}{l}\text { Refers to the route based construction heuristic combined with } \\
\text { the simulated annealing algorithm. }\end{array}$ \\
\hline PBFFS_SA & $\begin{array}{l}\text { Refers to the priority based construction heuristic combined } \\
\text { with the simulated annealing algorithm. }\end{array}$ \\
\hline LB & Lower bound \\
\hline
\end{tabular}




\subsection{Experimentation Overview}

Before applying the heuristics, the priority lists and the lower bounds are calculated using Microsoft Excel. A program was written in Microsoft Visual Basic-6.0 that takes the priority lists, job processing times, and setup times and calculates the solution for each heuristic. Also a VB program was written that performs all of the exchanges for the construction heuristics and displays the final sequence and the corresponding objective function. The program then inputs the final construction heuristic sequence and makespan as the starting sequence and makespan for the simulated annealing heuristic. The SA algorithm, also a VB program is initiated and runs until the temperature is reduced to a predetermined value.

\subsection{Simulated Annealing Parameters}

The simulated annealing parameters ensure that the temperature does not get reduced too fast, resulting in poor solutions. Also the SA parameters reduce the temperature fast enough that time is not wasted. The initial temperature is set by using the following equation:

$$
I T=\frac{(L B-S H 1)}{\ln (P A)}
$$

Where,

IT $=$ The initial temperature setting.

LB $\quad=$ Value obtained for the lower bound

SH1 = Value of the makespan found for the initial solution,

PA = Initial probability of accepting a non-improving solution, For this research the probability is set as 40 or $30 \%$.

The initial probability of accepting a non-improving solution is set at $40 \%$ for the first five runs (1-5) and set at 30\% for the next five runs (6-10). The value for the 
temperature reduction factor is set at 0.90 . The maximum number of iterations at each temperature setting is 100 for each problem. The heuristic is stopped when the temperature is reduced to 0.01 .

\subsection{Experimentation Results}

For each data set, the simple heuristic solutions and lower bounds were calculated first, and then the construction heuristics were initiated. Since the SA heuristic is random in nature and has a small run time it was run 10 different times. The PBFFS_SA heuristics run times were about 70, 95, and 135 seconds for the 3-1-2, 2-1-2-1, and 3-3-22-1 environments respectfully. The RBFFS_SA heuristics run times were all under 20 seconds for each environment. Each time the SA was run, it used the same starting solution given by the construction heuristic, but the initial probability of acceptance for a non-improving solution was adjusted. In the first five runs (1-5) the initial probability of acceptance was set at $40 \%$ and in the next five runs (6-10) the initial probability of acceptance was set at $30 \%$. In Tables 8.2 through 8.4 only the best solution obtained from the 10 runs is shown, all results for each run can be viewed in appendix A. In these tables, the ratio to LB is calculated as the ratio between the solution in question and LB, and the ratio to $\mathrm{BF}$ is calculated as the ratio between the solution in question and the best solution found.

For the 3-1-2 machine scheduling environment, the RBFFS heuristic averaged an increase of $23.2 \%$ from the lower bound, $14.8 \%$ from the best solution found, and achieved a 1.2\% improvement over the best simple heuristic solution. The RBFFS_SA heuristic averaged $7.3 \%$ from the lower bound, equaled the best solution found, and achieved a 16\% improvement over the best simple heuristic solution. Also by adding simulated annealing to RBFFS, the solution improved by $14.8 \%$. The PBFFS heuristic averaged $18.8 \%$ from the lower bound, $10.8 \%$ from the best solution found, and achieved a $4.7 \%$ improvement over the best simple heuristic solution. The PBFFS_SA heuristic averaged $12.1 \%$ from the lower bound, $4.5 \%$ from the best solution found, and achieved an $11 \%$ improvement over the best simple heuristic solution. By adding simulated annealing to PBFFS, the solution was improved by $6 \%$. 
For the 2-1-2-1 machine scheduling environment the RBFFS heuristic averaged $25.7 \%$ from the lower bound, $12 \%$ from the best solution found, and achieved a $1.7 \%$ improvement over the best simple heuristic solution. The RBFFS_SA heuristic averaged $12.3 \%$ from the lower bound, $0.1 \%$ from the best solution found, and achieved a $13.8 \%$ improvement over the best simple heuristic solution. Also by adding simulated annealing to RBFFS, the solution improved by $11.9 \%$. The PBFFS heuristic averaged $22.1 \%$ from the lower bound, $8.8 \%$ from the best solution found, and achieved a $4.7 \%$ improvement over the best simple heuristic solution. The PBFFS_SA heuristic averaged 15.3\% from the lower bound, $2.8 \%$ from the best solution found, and achieved a $10.8 \%$ improvement over the best simple heuristic solution. By adding simulated annealing to PBFFS, the solution was improved by $5.8 \%$.

For the 3-3-2-2-1 machine scheduling environment the RBFFS heuristic averaged $46 \%$ from the lower bound, $12.8 \%$ from the best solution found, and performed $3.6 \%$ worse than the best simple heuristic solution. The RBFFS_SA heuristic averaged 32.4\% from the lower bound, $2.3 \%$ from the best solution found, and $6.3 \%$ from the best simple heuristic solution. Also by adding simulated annealing to RBFFS, the solution improved by $10.3 \%$. The PBFFS heuristic averaged $36.2 \%$ from the lower bound, $5.2 \%$ from the best solution found, and achieved a 3.3\% improvement over the best simple heuristic solution. The PBFFS_SA heuristic averaged 29.5\% from the lower bound, equaled the best solution found, and achieved an $8.7 \%$ improvement over the best simple heuristic solution. By adding simulated annealing to PBFFS, the solution was improved by $5.2 \%$. 
Table 8-2 Experimentation Results for the 3-1-2 Environment

\begin{tabular}{|c|c|c|c|c|c|c|c|c|c|c|}
\hline \multirow[t]{3}{*}{ Configuration } & \multicolumn{10}{|c|}{ Buffer capacity $=3$} \\
\hline & & \multicolumn{3}{|c|}{$\mathrm{SH} 1$} & \multicolumn{3}{|c|}{$\mathrm{SH} 2$} & \multicolumn{3}{|c|}{ RBFFS } \\
\hline & LB & Sol & $\begin{array}{c}\text { Ratio to } \\
\text { LB }\end{array}$ & $\begin{array}{c}\text { Ratio to } \\
\text { BF }\end{array}$ & Sol & $\begin{array}{l}\text { Ratio } \\
\text { to LB }\end{array}$ & $\begin{array}{c}\text { Ratio to } \\
\text { BF }\end{array}$ & Sol & $\begin{array}{l}\text { Ratio } \\
\text { to LB }\end{array}$ & $\begin{array}{l}\text { Ratio } \\
\text { to BF }\end{array}$ \\
\hline Data set 1 & 205 & 275 & 1.3415 & 1.2387 & 265 & 1.2927 & 1.1937 & 266 & 1.2976 & 1.1982 \\
\hline Data set 2 & 228 & 285 & 1.25 & 1.1492 & 281 & 1.2325 & 1.1331 & 270 & 1.1842 & 1.0887 \\
\hline Data set 3 & 219 & 269 & 1.2283 & 1.1798 & 273 & 1.2466 & 1.1974 & 260 & 1.1872 & 1.1404 \\
\hline Data set 4 & 214 & 256 & 1.1963 & 1.1429 & 268 & 1.2523 & 1.1964 & 264 & 1.2336 & 1.1786 \\
\hline Data set 5 & 246 & 305 & 1.2398 & 1.1466 & 303 & 1.2317 & 1.1391 & 298 & 1.2114 & 1.1203 \\
\hline Data set 6 & 231 & 291 & 1.2597 & 1.1878 & 293 & 1.2684 & 1.1959 & 284 & 1.2294 & 1.1592 \\
\hline Data set 7 & 224 & 280 & 1.25 & 1.1864 & 274 & 1.2232 & 1.161 & 268 & 1.1964 & 1.1356 \\
\hline Data set 8 & 230 & 299 & 1.3 & 1.1818 & 290 & 1.2609 & 1.1462 & 303 & 1.3174 & 1.1976 \\
\hline Data set 9 & 258 & 322 & 1.2481 & 1.1709 & 324 & 1.2558 & 1.1782 & 301 & 1.1667 & 1.0945 \\
\hline Data set 10 & 199 & 253 & 1.2714 & 1.15 & 255 & 1.2814 & 1.1591 & 257 & 1.2915 & 1.1682 \\
\hline Average & 225.4 & 283.5 & 1.259 & 1.173 & 282.6 & 1.255 & 1.170 & 277.1 & 1.232 & 1.148 \\
\hline
\end{tabular}

\begin{tabular}{|l|c|c|c|c|c|c|c|c|c|c|}
\hline & & \multicolumn{3}{|c|}{ RBFFS_SA } & \multicolumn{3}{c|}{ PBFFS } & \multicolumn{3}{c|}{ PBFFS_SA } \\
\hline & LB & Sol & $\begin{array}{c}\text { Ratio to } \\
\text { LB }\end{array}$ & $\begin{array}{c}\text { Ratio to } \\
\text { BF }\end{array}$ & Sol & $\begin{array}{c}\text { Ratio } \\
\text { to LB }\end{array}$ & $\begin{array}{c}\text { Ratio to } \\
\text { BF }\end{array}$ & Sol & $\begin{array}{c}\text { Ratio } \\
\text { to LB }\end{array}$ & $\begin{array}{c}\text { Ratio } \\
\text { to BF }\end{array}$ \\
\hline Data set 1 & 205 & 222 & 1.0829 & 1 & 245 & 1.1951 & 1.1036 & 228 & 1.1122 & 1.027 \\
\hline Data set 2 & 228 & 248 & 1.0877 & 1 & 266 & 1.1667 & 1.0726 & 254 & 1.114 & 1.0242 \\
\hline Data set 3 & 219 & 228 & 1.0411 & 1 & 255 & 1.1644 & 1.1184 & 237 & 1.0822 & 1.0395 \\
\hline Data set 4 & 214 & 224 & 1.0467 & 1 & 254 & 1.1869 & 1.1339 & 239 & 1.1168 & 1.067 \\
\hline Data set 5 & 246 & 266 & 1.0813 & 1 & 292 & 1.187 & 1.0977 & 280 & 1.1382 & 1.0526 \\
\hline Data set 6 & 231 & 245 & 1.0606 & 1 & 279 & 1.2078 & 1.1388 & 261 & 1.1299 & 1.0653 \\
\hline Data set 7 & 224 & 236 & 1.0536 & 1 & 263 & 1.1741 & 1.1144 & 246 & 1.0982 & 1.0424 \\
\hline Data set 8 & 230 & 253 & 1.1 & 1 & 280 & 1.2174 & 1.1067 & 268 & 1.1652 & 1.0593 \\
\hline Data set 9 & 258 & 275 & 1.0659 & 1 & 303 & 1.1744 & 1.1018 & 287 & 1.1124 & 1.0436 \\
\hline Data set 10 & 199 & 220 & 1.1055 & 1 & 241 & 1.2111 & 1.0955 & 227 & 1.1407 & 1.0318 \\
\hline Average & 225.4 & 241.7 & 1.073 & 1 & 267.8 & 1.188 & 1.108 & 252.7 & 1.121 & 1.045 \\
\hline
\end{tabular}




\section{Table 8-3 Experimentation Results for the 2-1-2-1 Environment}

\begin{tabular}{|c|c|c|c|c|c|c|c|c|c|c|}
\hline \multirow[t]{3}{*}{ Configuration } & \multicolumn{4}{|c|}{$2-1-2-1$} & \multicolumn{3}{|c|}{ Buffer capacity $=3$} & \multirow{2}{*}{\multicolumn{3}{|c|}{ RBFFS }} \\
\hline & & & $\mathrm{SH}$ & & & $\mathrm{SH} 2$ & & & & \\
\hline & LB & Sol & $\begin{array}{l}\text { Ratio } \\
\text { to LB }\end{array}$ & $\begin{array}{c}\text { Ratio to } \\
\text { BF }\end{array}$ & Sol & $\begin{array}{c}\text { Ratio to } \\
\text { LB }\end{array}$ & $\begin{array}{c}\text { Ratio to } \\
\text { BF }\end{array}$ & Sol & $\begin{array}{l}\text { Ratio } \\
\text { to LB }\end{array}$ & $\begin{array}{c}\text { Ratio to } \\
\text { BF }\end{array}$ \\
\hline Data set 1 & 206 & 280 & 1.3592 & 1.1915 & 277 & 1.3447 & 1.1787 & 268 & 1.301 & 1.1404 \\
\hline Data set 2 & 237 & 310 & 1.308 & 1.2016 & 298 & 1.2574 & 1.155 & 284 & 1.1983 & 1.1008 \\
\hline Data set 3 & 225 & 294 & 1.3067 & 1.1713 & 296 & 1.3156 & 1.1793 & 289 & 1.2844 & 1.1514 \\
\hline Data set 4 & 214 & 277 & 1.2944 & 1.1688 & 278 & 1.2991 & 1.173 & 279 & 1.3037 & 1.1772 \\
\hline Data set 5 & 248 & 312 & 1.2581 & 1.1143 & 319 & 1.2863 & 1.1393 & 315 & 1.2702 & 1.125 \\
\hline Data set 6 & 234 & 307 & 1.312 & 1.1585 & 294 & 1.2564 & 1.1094 & 291 & 1.2436 & 1.0981 \\
\hline Data set 7 & 224 & 297 & 1.3259 & 1.1647 & 288 & 1.2857 & 1.1294 & 275 & 1.2277 & 1.0784 \\
\hline Data set 8 & 239 & 304 & 1.272 & 1.1259 & 307 & 1.2845 & 1.137 & 295 & 1.2343 & 1.0926 \\
\hline Data set 9 & 262 & 327 & 1.2481 & 1.1085 & 322 & 1.229 & 1.0915 & 323 & 1.2328 & 1.0949 \\
\hline Data set 10 & 216 & 276 & 1.2778 & 1.15 & 285 & 1.3194 & 1.1875 & 275 & 1.2731 & 1.1458 \\
\hline Average & 230.5 & 298.4 & 1.296 & 1.156 & 296.4 & 1.288 & 1.148 & 289.4 & 1.257 & 1.120 \\
\hline
\end{tabular}

\begin{tabular}{|c|c|c|c|c|c|c|c|c|c|c|}
\hline & & \multicolumn{3}{|c|}{ RBFFS_SA } & \multicolumn{3}{|c|}{ PBFFS } & \multicolumn{3}{|c|}{ PBFFS_SA } \\
\hline & LB & Sol & $\begin{array}{l}\text { Ratio } \\
\text { to LB }\end{array}$ & $\begin{array}{c}\text { Ratio to } \\
\text { BF }\end{array}$ & Sol & $\begin{array}{c}\text { Ratio to } \\
\text { LB }\end{array}$ & $\begin{array}{c}\text { Ratio to } \\
\text { BF }\end{array}$ & Sol & $\begin{array}{l}\text { Ratio } \\
\text { to LB }\end{array}$ & $\begin{array}{c}\text { Ratio to } \\
\text { BF }\end{array}$ \\
\hline Data set 1 & 206 & 235 & 1.1408 & 1 & 256 & 1.2427 & 1.0894 & 246 & 1.1942 & 1.0468 \\
\hline Data set 2 & 237 & 258 & 1.0886 & 1 & 280 & 1.1814 & 1.0853 & 267 & 1.1266 & 1.0349 \\
\hline Data set 3 & 225 & 251 & 1.1156 & 1 & 280 & 1.2444 & 1.1155 & 260 & 1.1556 & 1.0359 \\
\hline Data set 4 & 214 & 237 & 1.1075 & 1 & 267 & 1.2477 & 1.1266 & 245 & 1.1449 & 1.0338 \\
\hline Data set 5 & 248 & 280 & 1.129 & 1 & 302 & 1.2177 & 1.0786 & 290 & 1.1694 & 1.0357 \\
\hline Data set 6 & 234 & 265 & 1.1325 & 1 & 282 & 1.2051 & 1.0642 & 270 & 1.1538 & 1.0189 \\
\hline Data set 7 & 224 & 255 & 1.1384 & 1 & 276 & 1.2321 & 1.0824 & 259 & 1.1563 & 1.0157 \\
\hline Data set 8 & 239 & 270 & 1.1297 & 1 & 294 & 1.2301 & 1.0889 & 278 & 1.1632 & 1.0296 \\
\hline Data set 9 & 262 & 299 & 1.1412 & 1.0136 & 306 & 1.1679 & 1.0373 & 295 & 1.126 & 1 \\
\hline Data set 10 & 216 & 240 & 1.1111 & 1 & 267 & 1.2361 & 1.1125 & 247 & 1.1435 & 1.0292 \\
\hline Average & 230.5 & 259 & 1.123 & 1.001 & 281 & 1.221 & 1.088 & 265.7 & 1.153 & 1.028 \\
\hline
\end{tabular}


Table 8-4 Experimentation Results for the 3-3-2-2-1 Environment

Configuration
\begin{tabular}{|l|c|c|c|c|c|c|c|c|c|c|}
\hline & & \multicolumn{2}{|c|}{ S-3-2-2-1 } & \multicolumn{3}{c|}{ SH2 } & \multicolumn{3}{c|}{ RBFFS } \\
\hline & LB & Sol & $\begin{array}{c}\text { Ratio } \\
\text { to LB }\end{array}$ & $\begin{array}{c}\text { Ratio to } \\
\text { BF }\end{array}$ & Sol & $\begin{array}{c}\text { Ratio to } \\
\text { LB }\end{array}$ & Ratio to BF & Sol & $\begin{array}{c}\text { Ratio } \\
\text { to LB }\end{array}$ & $\begin{array}{c}\text { Ratio to } \\
\text { BF }\end{array}$ \\
\hline Data set 1 & 214 & 313 & 1.4626 & 1.0982 & 315 & 1.472 & 1.1053 & 320 & 1.4953 & 1.1228 \\
\hline Data set 2 & 243 & 334 & 1.3745 & 1.0503 & 348 & 1.4321 & 1.0943 & 351 & 1.4444 & 1.1038 \\
\hline Data set 3 & 226 & 331 & 1.4646 & 1.1336 & 333 & 1.4735 & 1.1404 & 338 & 1.4956 & 1.1575 \\
\hline Data set 4 & 222 & 305 & 1.3739 & 1.0517 & 319 & 1.4369 & 1.1 & 329 & 1.482 & 1.1345 \\
\hline Data set 5 & 264 & 363 & 1.375 & 1.0804 & 377 & 1.428 & 1.122 & 376 & 1.4242 & 1.119 \\
\hline Data set 6 & 247 & 348 & 1.4089 & 1.0943 & 353 & 1.4291 & 1.1101 & 361 & 1.4615 & 1.1352 \\
\hline Data set 7 & 239 & 329 & 1.3766 & 1.0894 & 352 & 1.4728 & 1.1656 & 346 & 1.4477 & 1.1457 \\
\hline Data set 8 & 243 & 355 & 1.4609 & 1.0957 & 355 & 1.4609 & 1.0957 & 365 & 1.5021 & 1.1265 \\
\hline Data set 9 & 268 & 378 & 1.4104 & 1.0957 & 379 & 1.4142 & 1.0986 & 384 & 1.4328 & 1.113 \\
\hline Data set 10 & 227 & 311 & 1.37 & 1.0799 & 339 & 1.4934 & 1.1771 & 322 & 1.4185 & 1.1181 \\
\hline Average & 239.3 & 336.7 & 1.408 & 1.087 & 347 & 1.451 & 1.121 & 349.2 & 1.460 & 1.128 \\
\hline
\end{tabular}

\begin{tabular}{|l|c|c|c|c|c|c|c|c|c|c|}
\hline & & \multicolumn{3}{|c|}{ RBFFS SA } & \multicolumn{3}{c|}{ PBFFS } & \multicolumn{3}{c|}{ PBFFS SA } \\
\hline & LB & Sol & $\begin{array}{c}\text { Ratio } \\
\text { to LB }\end{array}$ & $\begin{array}{c}\text { Ratio to } \\
\text { BF }\end{array}$ & Sol & $\begin{array}{c}\text { Ratio to } \\
\text { LB }\end{array}$ & Ratio to BF & Sol & $\begin{array}{c}\text { Ratio } \\
\text { to LB }\end{array}$ & $\begin{array}{c}\text { Ratio to } \\
\text { BF }\end{array}$ \\
\hline Data set 1 & 214 & 287 & 1.3411 & 1.007 & 297 & 1.3879 & 1.0421 & 285 & 1.3318 & 1 \\
\hline Data set 2 & 243 & 324 & 1.3333 & 1.0189 & 328 & 1.3498 & 1.0314 & 318 & 1.3086 & 1 \\
\hline Data set 3 & 226 & 297 & 1.3142 & 1.0171 & 326 & 1.4425 & 1.1164 & 292 & 1.292 & 1 \\
\hline Data set 4 & 222 & 294 & 1.3243 & 1.0138 & 296 & 1.3333 & 1.0207 & 290 & 1.3063 & 1 \\
\hline Data set 5 & 264 & 346 & 1.3106 & 1.0298 & 358 & 1.3561 & 1.0655 & 336 & 1.2727 & 1 \\
\hline Data set 6 & 247 & 328 & 1.3279 & 1.0314 & 330 & 1.336 & 1.0377 & 318 & 1.2874 & 1 \\
\hline Data set 7 & 239 & 312 & 1.3054 & 1.0331 & 323 & 1.3515 & 1.0695 & 302 & 1.2636 & 1 \\
\hline Data set 8 & 243 & 330 & 1.358 & 1.0185 & 342 & 1.4074 & 1.0556 & 324 & 1.3333 & 1 \\
\hline Data set 9 & 268 & 356 & 1.3284 & 1.0319 & 356 & 1.3284 & 1.0319 & 345 & 1.2873 & 1 \\
\hline Data set 10 & 227 & 295 & 1.2996 & 1.0243 & 302 & 1.3304 & 1.0486 & 288 & 1.2687 & 1 \\
\hline Average & 239.3 & 316.9 & 1.324 & 1.023 & 325.8 & 1.362 & 1.052 & 309.8 & 1.295 & 1.000 \\
\hline
\end{tabular}




\section{Conclusion}

\subsection{Overview of Research}

The objective of this research was to minimize the makepsan in a flexible flowshop with sequence dependent setup times, uniform machines, and limited buffers. At the time of this research, no attempt to solve this problem could be found in the literature. The goals of this research were to:

1. Develop a mathematical model of the problem.

2. Develop two construction heuristics to construct initial feasible solutions to the problem. Then use a simulated annealing algorithm to improve the initial solutions.

3. Develop a lower bound to test the solution quality.

This problem is extremely complex and it is not practical to solve optimally. A mathematical model for the problem was created, and used to solve small problems optimally. Even very small problems, such as a three stage 5 job problem, took around an hour to solve.

Two construction heuristics, RBFFS and PBFFS were developed based on a flowshop heuristic given by Nawaz et. al (1983). The first heuristic is route based, and the second is job priority based. The heuristics give a higher scheduling priority to jobs that have larger processing times. They use adjacent pariwise exchange and add jobs one at a time until all jobs are scheduled. Next, a simulated annealing heuristic is used to improve the final solutions obtained from the construction heuristics. The construction and SA heuristics use a solution space of $n$ !, where $n$ is the total number of jobs. This reduces the solution space but allows good solutions to be found in a reasonable amount of time. 
Two methods were used to test the proposed algorithms performance. A lower bound was developed that focused on the bottleneck stage and then scheduled jobs before and after that stage. Also two simple heuristics were used to solve the problem. One simple heuristic, SH1, assigned jobs priority on a first come first serve basis. The second simple heuristic, $\mathrm{SH} 2$, assigned jobs priorities based on total processing time, with the higher total processing time jobs receiving higher priority. The simple heuristics provide feasible solutions that are used for comparison.

In all but one case, both construction heuristics obtained a better solution than the best simple heuristic. The simulated annealing heuristic was able to improve on the solutions obtained with the construction heuristics. When comparing to the lower bound, both construction algorithms and simulated annealing heuristics performed well for the smaller systems. For the machine configurations of 3-1-2, 2-1-2-1 and 3-3-2-2-1, the RBFFS_SA heuristic was 7.3\%, 12.3\%, and 32.4.5\% respectively higher than the lower bound; while the PBFFS_SA heuristic was $12.1 \%, 15.3 \%$, and $29.5 \%$ higher than the lower bound. The increase in the gap between the heuristic solution and the lower bound for the larger stage environments is most likely due to deficiencies in the lower bound and not due to poor heuristic performance. The presented lower bound does not account for machine blocking, and as the number of stages increase, the chances of a machine being blocked also increase. The lower bound presented was compared to a lower bound calculated by relaxing the binary and integer variables. The LB presented outperformed the relaxed version.

The RBFFS_SA heuristic performed the best for the small and medium sized problems. The PBFFS_SA heuristic out performed the RBFFS for the larger problem. 
The simulated annealing algorithm had a much larger affect on the RBFFS's solution; it was improved between $10.3 \%$ and $14.8 \%$, while the SA improved the PBFFS only $5.2 \%$ to $6 \%$. Limiting the solution space to permutation schedules may have produced this difference. The RBFFS is much more delicate and dependant on the permutation solution. The RBFFS_SA heuristic has a short run time, but it does take extra time to develop a good routing system. Both heuristics were able to provide good solutions to the problem in a reasonable amount of time.

\subsection{Contribution to Literature:}

The contributions made to the literature by this research are as follows:

- A mathematical model was developed for the flexible flowshop with sequence dependent setup times, uniform machines, and limited buffers.

- Development of two construction heuristics combined with an improvement heuristic to give a good quick solution to the problem.

- Development of a simulated annealing heuristic to improve the solution of the construction heuristics.

- Development of a lower bound for the problem.

- Laid ground work for future research on this type of problem.

\subsection{Recommendations for Future Research:}

The work performed in this research opens the door for more investigation of the flexible flowshop with sequence dependent setup times, uniform machines, and limited buffers problem. Suggestion for future research include: 
- Develop a tighter lower bound to test the heuristic approaches to this problem by adding a factor to account for machine blocking.

- Look at other meta-heuristics to improve the solution obtained from the construction algorithm.

- Experiment with different job priority systems for the problem.

- Experiment with other machine configurations and/or different systems of data generation.

- Add job ready times and/or due dates.

- Before scheduling a job check to see which machine would finish processing it faster. The total time may be less if it waits for a machine to open up, instead of being processed on the first available machine. 


\section{References}

Acero-Dominguez, M. and Paternina-Arboleda, C. (2004). Scheduling Jobs on a K-Stage Flexible Flowshop Using a TOC-Based (Bottleneck) Procedure. Proceedings of the 2004 Systems and Information Engineering Design Symposium, 295-297.

Adams, J., Balas, E., and Zawack, D. (1988). The Shifting Bottleneck Procedure For Job Shop Scheduling. Management Science, 34 (3), 391-401.

Agnetis, A., Rossi, F., and Gristina, G. (1998). An Exact Algorithm for the Batch Sequence Problem in a Two-Machine Flowshop with Limited Buffer. Naval Research Logistics, 45, 141-164.

Allahverdi, A. (2000). Minimizing mean flow time in a two-machine flowshop with sequence-independent setup times. Computers and Operations Research, 111-127.

Allahverdi, A. and Aldowaisan, T. (2004). No-wait flowshops with bicriteria of makespan and maximum lateness. European Journal of Operational Research, 152, 132-147.

Allahverdi, A., Gupta, J.N.D., and Aldowaisan, T. (1999). A review of scheduling research involving setup considerations. Omega International Journal of Management Science, 27, 219-239.

Arthanari, T., and Ramamurthy, K. (1971). An Extension of Two Machines Sequencing Problem. Opsearch, 8, 10-22.

Azizoglu, M., Cakmak, E., and Suna, K. (2001). A flexible flowshop problem with total flow time minimization. European Journal of Operational Research, 132, 528-538.

Baker, K. (1974). Introduction to Sequencing \& Scheduling. United States: John Wiley \& Sons.

Barman, S. (1998). The impact of priority rule combinations on lateness and tardiness. IIE Transactions, 30, 495-504.

Brah, S. and Hunsucker, J. (1991). Branch and bound algorithm for the flowshop with multiple processors. European Journal of Operational Research 2 51, 88-99.

Brah, S. and Loo, L. (1999). Heuristics for scheduling in a flowshop with multiple processors. European Journal of Operational Research, 113, 113-122.

Buzacott, J. and Yao, D. (1986). Flexible Manufacturing Systems: A Review of Analytical Models. Management Science, 32 (7), 890-905. 
Campbell, H., Dudek, R., and Smith, M. (1970). A Heuristic Algorithm for the $n$ Job, $m$ Machine Sequencing Problem. Management Science, 16(10), B-630-37.

Carlier, Jacques (1982). The one-machine sequencing problem. European Journal of Operational Research, 11, 42-47.

Carlier, J. and Rebai, I. (1996). Two branch and bound algorithms for the permutation flowshop problem. European Journal of Operational Research, 90, 238-251.

Chang, S. (1994). Scheduling Flexible Flowshops with No Setup Effects. IEEE Transactions on Robotics and Automation, 10(2), 112-122.

Chen, Bo., Glass, C., Potts, C., and Strusevich V. (1996). A New Heuristic for ThreeMachine Flowshop Scheduling. Operations Research. 44(6), 891-898.

Chen, Bo (1995). Analysis of Classes of Heuristics for Scheduling a Two-Stage Flowshop with Parallel Machines at One Stage. Journal of the Operational Research Society, 46, 234-244.

Chen, Bo (1994). Scheduling multiprocessor flowshops. Advances in optimization and approximation, 1-8.

Cheng, J., Karuno, Y. and Kise, H. (2001). A shifting bottleneck approach for a parallelmachine flowshop scheduling problem. Journal of the Operations Research Society of Japan, 44(2), 140-156.

Cheng, T., and Sin, C. (1990). A state-of-the-art review of parallel-machine scheduling research. European Journal of Operational Research 2 47, 271-292.

Cliffe, R., and MacManus, B. (1980). An approach to optimization with heuristic methods scheduling. International Journal of Production Research, 18(4), pp 479-490.

Co, Henry (1992). Streamlining material flow in flexible manufacturing systems: a lesson in simplicity. International Journal of Production Research, 32(7), 1483-1499.

Conway, R., Maxwell, W., \& Miller, L. (1967). Theory of Scheduling. New York: Dover Publications.

Corwin, B. and Esogbue, A. (1974). Two Machine Flowshop Scheduling Problems with Sequence Dependent Setup Times: A Dynamic Programming Approach. Naval Research Logistics Quarterly, 21(3), 515-524.

Daniels, R. and Mazzola, J. (1994). Flowshop Scheduling With Resource Flexibility. Operations Research Society of America, 42(3), 504-522. 
Dannenbring, D. (1977). An Evaluation of Flowshop Sequencing Heuristics.

Management Science , 23(11), 1174-112

Day, J., and Hottenstein, M. (1970). Review of Sequencing Research. Naval Research Logistics Quarterly, 17(1), 11-39.

Ding, F., and Kittichartphayak, D. (1994). Heuristics For Scheduling Flexible Flow Lines. Computers and Industrial Engineering, 26(1), 27-34.

Dudek, R., Panwalkar, S., and Smith, M. (1992). The Lessons of Flowshop Scheduling Research. Operations Research, 40(1), 7-13.

Eglese, R. (1990). Simulated Annealing: A tool for operational research. European Journal of Operational Research, 46, 271-281.

Garey, M., Johnson, D., and Sethi, R. (1976). The Complexity of Flowshop and Jobshop Scheduling. Mathematics of Operations Research, 1(2), 117-129.

Gilmore, P., and Gomory, R. (1964). Sequencing a One State-Variable Machine a Solvable Case of the Traveling Salesman Problem. Operations Research, 12, 655-679.

Gonzalez, T., Ibarra, O., and Sahni, S. (1977). Bounds for LPT Schedules on Uniform Processors. SIAM Journal on Computing, 6(1), 155-166.

Guinet, A. and Solomon, M. (1996). Scheduling hybrid flowshops to minimize maximum tardiness or maximum completion time. International Journal of Production Research, 34(6), 1643-1654.

Guinet, A., Solomon, M., Kedia, P., and Dussauchy, A. (1996). A computational study of heuristics for two-stage flexible flowshops. International Journal of Production Research, 34(5), 1399-1415.

Gupta, J.N.D. (1972). Heuristic algorithms for multistage flowshop scheduling problem. AIIE Transactions, 4(1), 11-18.

Gupta, J.N.D. (1976). Optimal Flowshop Schedules with No Intermediate Storage Space. Naval Research Logistics Quarterly, 23, 235-243.

Gupta, J.N.D. (1986). Flowshop Schedules with Sequence Dependent Setup Times. Journal of the Operations Research Society of Japan, 29(3), 206-219.

Gupta, J.N.D. (1988). Two-Stage, Hybrid Flowshop Scheduling Problem. Operational Research Society, 39(4), 359-364.

Gupta, J.N.D., Hariri, A., and Potts, C. (1997). Scheduling a two-stage hybrid flowshop with parallel machines at the first stage. Annals of Operations Research, 69, 171-191. 
Gupta, JND and Ruiz-Torres, A. (2000). Minimizing makespan subject to minimum total flow-time on identical parallel machines. European Journal of Operational Research, $125,370-380$.

Gupta, J.N.D., and Szwarc, W. (1987). A Flow-Shop Problem with Sequence-Dependent Additive Setup Times. Naval Research Logistics, 34, 619-627.

Gupta, J.N.D., and Tunc, E. (1994). Scheduling a two-stage hybrid flowshop with separable setup and removal times. European Journal of Operational Research, 77, 415-428.

Gupta, J.N.D., and Tunc, E. (1991). Schedules for a two-stage hybrid flowshop with parallel machines at the second stage. International Journal of Production Research, 29(7), 1489-1502.

Gupta, Sushil (1982). N jobs and M machines job-shop problems with sequencedependent setup times. International Journal of Production Research, 20, (5), 643656.

Haouari, M., and M'Hallah, R. (1997). Heuristic algorithms for the two-stage hybrid flowshop problem. Operations Research Letters, 21, 43-53.

Hejeck, B. (1988). Cooling Schedules for optimal annealing. Mathematics of Operations Research, 13(2), 311-329.

Ho, J. (1995). Flowshop sequencing with mean flowtime objective. European Journal of Operational Research, 81, 571-578.

Ho, J., and Chang, Y. (1991). A new heuristic for the n-job, M-machine flow-shop problem. European Journal of Operational Research, 52, 194-202.

Hoogeveen, J., Lenstra, J, and Veltman, B. (1996). Preemptive scheduling in a two-stage multiprocessor flowshop is NP-hard. European Journal of Operational Research, 89, 172-175.

Huang, W. (1998). A Two-Stage Hybrid Flowshop with Uniform Machines and Setup Times. Mathematical and Computer Modelling, 27(2), 27-45.

Hundal, T., and Rajgopal, J. (1988). An extension of Palmer's heuristic for the flowshop scheduling problem. International Journal of Production Research, 26(6), 1119-1124.

Ishibuchi, H., Misaki, S., and Tanaka, H. (1995). Modified simulated annealing algorithms for the flowshop sequencing problem. European Journal of Operational Research, 81, 388-398. 
Ignall, E. and Schrage, L. (1965). Application of the Branch-and-Bound Technique to Some Flow-Shop Scheduling Problems. Operations Research, 13(3)

Jayamohan, M. and Rajendran, C. (2000). A comparative analysis of two different approaches to scheduling in flexible flowshops. Production Planning and Control, 11(6), 572-580.

Johnson, S.M. (1954). Optimal Two-and Three-Stage Production Schedules with Setup Times included. Naval Research Logistics Quarterly, 1(1), 61-67.

Kadipasaoglu, S., Xiang, W., and Khumawala, B. (1997). A comparison of sequencing rules in static and dynamic hybrid flow systems. International Journal of Production Research, 35(5), 1359-1384.

Khmelnitsky, E., Kogan, K., and Maimon, O. (1997). Maximum principle-based methods for production scheduling with partially sequence-dependent setups.

International Journal of Production Research, 35(10), 2701-2712.

King, J. and Spachis, A. (1980). Heuristic for flow-shop scheduling. International Journal of Production Research, 18(3), 345-357.

Kirkpatrick, S., Gelatt, D., and Vecchi, M. (1983). Optimization by Simulated Annealing. Science, 220 (4598).

Kochhar, S. and Morris, R. (1987). Heuristic Methods for Flexible Flow Line Scheduling. Journal of Manufacturing Systems, 6(4), 299-314.

Kochhar, S., Morris, R., and Wong, W. (1988). The Local Search Approach to Flexible Flow Line Scheduling. Engineering Costs and Production Economics, 14, 25-37.

Koulamas, C. (1998). A new constructive heuristic for the flowshop scheduling problem. European Journal of Operational Research, 105, 66-71.

Koulamas, C., and Kyparisis, G. (2000). Asymptotically Optimal Linear Time Algorithms for Two-Stage and Three-Stage Flexible Flowshops. Naval Research Logistics , 47, 259-268.

Kouvelis, P., and Chiang, W. (1992). A simulated annealing procedure for single row layout problems in flexible manufacturing systems. International Journal of Production Research, 30(4), 717-732.

Lawler, E. et al. (1993). Sequencing and Scheduling: Algorithms and Complexity. Handbook in Operations Research and Management Science, Edited by Rinnooy Kan and Zipkin, Vol. 4: Logistics of Production and Inventory, pp 445-522. North-Holland, New York. 
Lee, C., Cheng, T., and Lin, B. (1993). Minimizing the Makespan in the 3-Machine Assembly-type Flowshop Scheduling Problem. Management Science, 39(5), 616-625.

Lee, C. and Vairaktarakis, G. (1994). Minimizing makespan in hybrid flowshops. Operations Research Letters, 16, 149-158.

Lee, C. and Vairaktarakis, G. (1998). Performance Comparison of Some Classes of Flexible Flowshops and Job Shops. The International Journal of Flexible Manufacturing Systems, 10, 379-405.

Lee, G., Kin, Y., and Choi, S. (2004). Bottleneck-focused scheduling for a hybrid flowshop. International Journal of Production Research, 42(1), 165-181.

Lee, Y. and Pinedo, M. (1997). Scheduling jobs on parallel machines with sequencedependent setup times. European Journal of Operational Research, 100, 464-474.

Leon, V., and Ramamoorthy, B. (1997). An adaptable problem-space-based search method for flexible flow line scheduling. IIE Transactions, 29, 115-125.

Li, Shanling (1997). A hybrid two-stage flowshop with part family, batch production major and minor setups. European Journal of Operational Research, 102, 142-156.

Linn, R. and Zhang, W. (1999). Hybrid Flowshop Scheduling: A Survey. Computers \& Industrial Engineering, 37, 57-61.

Liu, C., and Chang, S. (2000). Scheduling Flexible Flowshops with SequenceDependent Setup Effects. IEEE Transactions on Robotics and Automation, 16(4), 408419.

Loukil, T., Teghem, J., and Tuyttens, D. (2005). Solving multi-objective production scheduling problems using meta-heuristics. European Journal of Operational Research, $161,42-61$.

Low, C. (2005). Simulated annealing heuristic for flowshop scheduling problems with unrelated parallel machines. Computers \& Operations Research, 32, 2013-2025.

Low, C., Yeh, J., and Huang, K. (2004). A robust simulated annealing heuristic for flowshop scheduling problems. International Journal of Advanced Manufacturing Technology, 23, 762-767.

Moursli, O. (1995). Branch and Bound Lower Bounds for the Hybrid Flowshop. Intelligent Manufacturing Systems , $4^{\text {th }}$ IFAC Workshop, 31-36.

Narasimham, S. and Mangiameli, P. (1987). A Comparison of Sequencing Rules for a Two-Stage Hybrid Flowshop. Decision Sciences, 18, 250-265. 
Narasimhan, S., and Panwalker, S. (1984). Scheduling in a two-stage manufacturing process. International Journal of Production Research, 22(4), 555-564.

Nawaz, M., Enscore, E., and Ham, I. (1983). A Heuristic Algorithm for the m-machine n-Job Flowshop Sequencing Problem. Omega, 11(1), 91-95.

Nearchou, A. (2004). A novel meta-heuristic approach for the flowshop scheduling problem. Engineering Applications of Artificial Intelligence, 17, 289-300.

Nearchou, A. (2004). Flow-shop sequencing using hybrid simulated annealing. Journal of Intelligent Manufacturing, 15, 317-328.

Negenman, E. (2001). Local search algorithms for the multiprocessor flowshop scheduling problem. European Journal of Operational Research, 128, 147-158.

Neron, E., Baptiste, P., and Gupta, JND. (2001). Solving hybrid flowshop problem using energetic reasoning and global operations. Omega, 29, 501-511.

Norman, B. (1999). Scheduling flowshops with finite buffers and sequence-dependent setup times. Computers \& Industrial Engineering, 36, 163-177.

Nowicki, E., and Smutnicki, C. (1993). New results in the worst-case analysis for flowshop scheduling. Discrete Applied Mathematics, 46, 21-41.

Nowicki, E., and Smutnicki, C. (1989). Worst-Case Analysis of an Approximation Algorithm for Flow-Shop Scheduling. Operations Research Letters, 8(3), 171-177.

Ogbu, F. and Smith, D. (1990). Simulated Annealing for the Permutation Flowshop Problem. Omega, 19(1), 64-67.

Ogbu, F. and Smith, D. (1990). The application of the simulated annealing algorithm to the solution of the $\mathrm{n} / \mathrm{mCmax}$ flowshop problem. Computers and Operations Research, $17(3), 243-253$.

Oguz, C., Lin, B., and Cheng, T. (1997). Two-stage flowshop scheduling with a common second-stage machine. Computers and Operations Research, 24(12), 11691174.

Oi, Mei. (1996). Scheduling two-stage production lines with multiple machines. Production Planning \& Control, 7(4), 418-429

Osman, I., and Potts, C. (1989). Simulated Annealing for Permutation Flow-Shop Scheduling. OMEGA International Journal of Management Science, 17(6), 551-557. 
Palmer, D. (1965). Sequencing Jobs through a Multi-Stage Process in the Minimum Total Time-A Quick Method of Obtaining a Near Optimum. Operations Research Quarterly, $16,101-107$.

Parthasarathy, S. and Rajendran, C. (1997). A simulated annealing heuristic for scheduling to minimize mean weighted tardiness in a flowshop with sequence-dependent setup times of jobs- a case study. Production Planning and Control, 8(5). 475-483.

Peeters, P., Brussel, H., Valckenaers, P., Wyns, Jo., Bongaerts, L., Kollingbaum, M., and Heikkila, T. (2001). Pheromone based emergent shop floor control system for flexible flowshops. Artificial Intelligence in Engineering, 15, 343-352.

Phadnis, S., Brevick, J., and Irani, S. (2003, March). Development of a New Heuristic For Scheduling Flow-Shops with Parallel Machines By Prioritizing Bottleneck Stages. Transactions of the Society for Design and Process Science, 7(1), 87-97.

Pinedo, M., (2002). Scheduling Theory, Algorithms, and Systems. New Jersey: Prentice Hall.

Portman, M., Vignier, A., Dardilhac, D., and Dezalay, D. (1998). Branch and bound crossed with GA to solve hybrid flowshops. European Journal of Operational Research, $107,389-400$.

Raban, S., and Nagel, R. (1991). Constraint-based control of flexible flow lines. International Journal of Production Research, 29(10), 1941-1951.

Rajendran, C., and Chaudhuri, D. (1992). Scheduling in n-job, m-stage flowshop with parallel processors to minimize makespan. International Journal of Production Economics, 27, 137-143.

Randhawa, S., and Kuo, C. (1997). Evaluating scheduling heuristics for non-identical parallel processors. International Journal of Production Research, 35(4), 969-981.

Randhawa, S,. and Smith, T. (1995). An experimental investigation of scheduling nonidentical, parallel processors with sequence-dependent setup times and due dates. International Journal of Production Research, 33(1), 59-69.

Riane, F., Artiba, A., and Elmaghraby, S. (1998). A hybrid three-stage flowshop problem: Efficient heuristics to minimize makespan. European Journal of Operational Research, 109, 321-329.

Riane, F., Raczy, C., and Artiba, A. (1999). Hybrid Auto-adaptable Simulated Annealing based Heuristic. Computers \& Industrial Engineering, 37, 277-280. 
Riane, F.k, Artiba, A., and Elamaghraby, S. (2002). Sequencing a hybrid two-stage flowshop with dedicated machines. International Journal of production Research, $40(17), 4353-4380$.

Rios-Mecado, R. and Bard, J. (1999). A branch-and-bound algorithm for permutation flowshops with sequence-dependent setup times. IIE Transactions, 31, 721-731.

Rios-Mercado, R. and Bard, J. (1999). An Enhanced TSP-Based Heuristic for Makespan Minimization in a Flowshop with Setup Times. Journal of Heuristics, 5, 53-70.

Rios-Mercado, R. and Bard, J. (2003). The Flowshop Scheduling Polyhedron with Setup Times. Journal of Combinatorial Optimization, 7, 291-318.

Rios-Mercado, R. and Bard, J. (1998). Heuristics for the flow line problem with setup costs. European Journal of Operational Research, 110, 76-98.

Sarin, S. and Lefoka, M. (1993). Scheduling Heuristic for the n-Job m-Machine Flowshop. OMEGA International Journal of Management Science, 21(2), 229-234.

Sawik, T. (2002). An Exact Approach for Batch Scheduling in Flexible Flow Lines with Limited Intermediate Buffers. Mathematical and Computer Modelling, 36, 461-471.

Sawik, T. (2000). Mixed Integer Programming for Scheduling Flexible Flow Lines with Limited Intermediate Buffers. Mathematical and Computer Modelling, 31, 39-52.

Sawik, T. (1995). Scheduling flexible flow lines with no in-process buffers. International Journal of Production Research, 33(5), 1357-1367.

Sawik, T. (1987). Multilevel Scheduling of Multistage Production with Limited InProcess Inventory. Journal of the Operational Research Society, 38(7), 651-664.

Sethanan, K. (2001). Scheduling Flexible Flowshops with Sequence Dependent Setup Times. Dissertation for Doctor of Philosophy in Decision Sciences and Production Systems, West Virginia University.

Shieh, A. (2003). A Simulated Annealing Approach for Flexible Flowshop Scheduling to Maximize Flexiblity. Masters Thesis, for Master of Science in Industrial Engineering, West Virginia University.

Simons, J. (1992). Heuristics in Flowshop Scheduling with Sequence Dependent Setup Times. OMEGA International Journal of Management Science, 20(2), 215-225.

Soewandi, H., and Elmaghraby, S. (2003). Sequencing on two-stage hybrid flowshops with uniform machines to minimize makespan. IIE Transactions, 35, 467-477. 
Soewandi, H., and Elmaghraby, S. (2001). Sequencing three-stage flexible flowshops with identical machines to minimize makespan. IIE Transactions, 33, 985-993.

Srikar, B. and Ghosh, S. (1986). A MILP model for the n-job, M-stage flowshop with sequence dependent setup times. International Journal of Production Research, 24(6), $1459-1474$.

Sriskandarajah, C., and Sethi, S. (1989). Scheduling algorithms for flexible flowshops: Worst and average case performance. European Journal of Operational Research, 43, 143-160.

Stafford, E., and Tseng, F. (1990). On the Srikar-Ghosh MILP model for the N x M SDST flowshop problem. International Journal of Production Research, 28(10), 18171830.

Stecke, K. and Kim, I. (1991). A flexible approach to part type selection in flexible flow systems using part mix ratios. International Journal of Production Research, 29(1), 5375.

Szwarc, W. and Gupta, J. (1987). A Flow-Shop Problem with Sequence-Dependent Additive Setup Times. Naval Research Logistics, 34, 619-627.

Taillard, E. (1993). Benchmarks for basic scheduling problems. European Journal of Operational Research, 64, 278-285.

Taillard, E. (1990). Some efficient heuristic methods for the flowshop sequencing problem. European Journal of Operational Research, 47, 65-74.

Townsend, D. (1977). Sequencing $n$ jobs on $m$ machines to minimize tardiness: a branch and bound solution. Management Science, 23, 1016-1019.

Tsubone, H., Ohba, M., and Uetake, T. (1996). The impact of lot sizing and sequencing on manufacturing performance in a two-stage hybrid flowshop. International Journal of Production Research, 34(11), 3037-3053.

Turner, S. and Booth, D. (1987). Comparison of Heuristics for Flowshop Sequencing. Omega, 15(1), 75-85.

Uetake, T., Tsubone, H., and Ohba, M. (1995). A production scheduling system in a hybrid flowshop. International Journal of Production Economics, 41, 395-398.

Verma, S., and Dessouky, M. (1999). Multistage Hybrid Flowshop Scheduling With Identical Jobs and Uniform Parallel Machines. Journal of Scheduling, 2, 135-150.

Vignier, A., Dardilhac, D., Dezalay, D., and Proust, C. (1996). A branch and bound approach to minimize the total completion time in a k-stage hybrid flowshop. 
Procedings: IEEE Conference on Emerging Technologies and Factory Automation 2215 $-220$.

Wang, C., Chu, C., and Proth, J. (1997). Heuristic approaches for $n / m / F / \Sigma C i$ scheduling problems. European Journal of Operational Research, 96, 636-644.

Wang, H., Jacob, V., and Rolland, E. (2003). Design of efficient hybrid neural networks for flexible flowshop scheduling. Expert Systems, 20(4), 208-231.

Wang, L. and Zheng, D. (2003). An Effective Hybrid Heuristic for Flowshop Scheduling. International Journal of Manufacturing Technology, 21, 38-44.

Weng, M. (2000). Scheduling Flow-Shops with Limited Buffer Spaces. Proceedings of the 2000 Winter Simulation Conference, 1359-1363.

White, C., and Wilson, R. (1977). Sequence dependent setup times and job sequencing. International Journal of Production Research, 15(2), 191-202.

Widmer, M. and Hertz, A. (1989). A new heuristic method for the flowshop sequencing problem. European Journal of Operational Research, 41, 186-193.

Wittrock, R. (1988). An Adaptable Scheduling Algorithm for Flexible Flow Lines. Operations Research Society of America, 36(3), 445-453

Wittrock, R. (1985). Scheduling algorithms for flexible flow lines. IBM Journal of Research and Development, 29(4), 401-412.

Ying, K. and Liao, C. (2004). An ant colony system for permutation flow-shop sequencing. Computers an Operations Research, 31, 791-801.

Zegordi, S., Itoh, K., and Enkawa, T. (1995). Minimizing makespan for flowshop scheduling by combining simulated annealing with sequencing knowledge. European Journal of Operational Research, 85, 515-531.

Zhou, C. and Egbelu, P. (1989). Scheduling in a manufacturing shop with sequencedependent setup times. Robotics and Computer-Integrated Manufacturing, 5(1), 73-81. 


\section{Appendix A: Experimental Results}

\begin{tabular}{|c|c|c|c|c|c|c|c|c|c|}
\hline \multicolumn{2}{|c|}{ Data set 1} & \multirow[t]{2}{*}{ Ratio to LB } & \multirow{2}{*}{\multicolumn{2}{|c|}{$\begin{array}{c}\text { Stage Configuration } \\
3-1-2 \\
\end{array}$}} & \multicolumn{2}{|c|}{ Data set 2} & \multirow[t]{2}{*}{ Ratio to LB } & \multirow{2}{*}{\multicolumn{2}{|c|}{$\begin{array}{c}\text { Stage Configuration } \\
3-1-2 \\
\end{array}$}} \\
\hline LB & 205 & & & & LB & 228 & & & \\
\hline $\mathrm{SH} 1$ & 275 & 1.3415 & \multicolumn{2}{|c|}{ Buffers size $=3$} & $\mathrm{SH} 1$ & 285 & 1.2500 & \multicolumn{2}{|c|}{ Buffers size $=3$} \\
\hline $\mathrm{SH} 2$ & 265 & 1.2927 & & & $\mathrm{SH} 2$ & 281 & 1.2325 & & \\
\hline RBFFS & 266 & 1.2976 & & & RBFFS & 270 & 1.1842 & & \\
\hline $\begin{array}{l}\text { Data set } \\
1\end{array}$ & RBFFS_SA & $\begin{array}{l}\text { Ratio } \\
\text { RBFFS_SA } \\
\text { to LB }\end{array}$ & $\begin{array}{l}\text { Ratio Best } \\
\text { SH to } \\
\text { RBFFS_SA }\end{array}$ & $\begin{array}{c}\text { Ratio } \\
\text { RBFFS to } \\
\text { RBFFS_SA }\end{array}$ & $\begin{array}{l}\text { Data set } \\
2\end{array}$ & RBFFS_SA & $\begin{array}{c}\text { Ratio } \\
\text { RBFFS_SA } \\
\text { to LB }\end{array}$ & $\begin{array}{c}\text { Ratio Best } \\
\text { SH to } \\
\text { RBFFS_SA }\end{array}$ & $\begin{array}{c}\text { Ratio } \\
\text { RBFFS to } \\
\text { RBFFS_SA }\end{array}$ \\
\hline Exp 1 & 223 & 1.0878 & 1.1883 & 1.1928 & Exp 1 & 248 & 1.0877 & 1.1331 & 1.0887 \\
\hline Exp 2 & 229 & 1.1171 & 1.1572 & 1.1616 & Exp 2 & 251 & 1.1009 & 1.1195 & 1.0757 \\
\hline Exp 3 & 226 & 1.1024 & 1.1726 & 1.1770 & Exp 3 & 249 & 1.0921 & 1.1285 & 1.0843 \\
\hline Exp 4 & 222 & 1.0829 & 1.1937 & 1.1982 & Exp 4 & 252 & 1.1053 & 1.1151 & 1.0714 \\
\hline Exp 5 & 225 & 1.0976 & 1.1778 & 1.1822 & Exp 5 & 249 & 1.0921 & 1.1285 & 1.0843 \\
\hline Exp 6 & 226 & 1.1024 & 1.1726 & 1.1770 & Exp 6 & 254 & 1.1140 & 1.1063 & 1.0630 \\
\hline $\operatorname{Exp} 7$ & 225 & 1.0976 & 1.1778 & 1.1822 & Exp 7 & 249 & 1.0921 & 1.1285 & 1.0843 \\
\hline Exp 8 & 230 & 1.1220 & 1.1522 & 1.1565 & Exp 8 & 248 & 1.0877 & 1.1331 & 1.0887 \\
\hline Exp 10 & 231 & 1.1268 & 1.1472 & 1.1515 & Exp 10 & 252 & 1.1053 & 1.1151 & 1.0714 \\
\hline
\end{tabular}




\begin{tabular}{|c|c|c|c|c|c|c|c|c|c|}
\hline \multicolumn{2}{|c|}{ Data set 3} & Ratio to LB & \multirow{2}{*}{\multicolumn{2}{|c|}{$\begin{array}{c}\text { Stage Configuration } \\
3-1-2 \\
\end{array}$}} & \multicolumn{2}{|c|}{ Data set 4} & Ratio to LB & \multirow{2}{*}{\multicolumn{2}{|c|}{$\begin{array}{c}\text { Stage Configuration } \\
3-1-2 \\
\end{array}$}} \\
\hline LB & 219 & & & & LB & 214 & & & \\
\hline $\mathrm{SH} 1$ & 269 & 1.2283 & \multicolumn{2}{|c|}{ Buffers size $=3$} & $\mathrm{SH} 1$ & 256 & 1.1963 & \multicolumn{2}{|c|}{ Buffers size $=3$} \\
\hline $\mathrm{SH} 2$ & 273 & 1.2466 & & & $\mathrm{SH} 2$ & 268 & 1.2523 & & \\
\hline RBFFS & 260 & 1.1872 & & & RBFFS & 264 & 1.2336 & & \\
\hline $\begin{array}{l}\text { Data set } \\
3\end{array}$ & RBFFS_SA & $\begin{array}{l}\text { Ratio } \\
\text { RBFFS_SA } \\
\text { to LB }\end{array}$ & $\begin{array}{l}\text { Ratio Best } \\
\text { SH to } \\
\text { RBFFS_SA }\end{array}$ & $\begin{array}{c}\text { Ratio } \\
\text { RBFFS to } \\
\text { RBFFS_SA }\end{array}$ & $\begin{array}{l}\text { Data set } \\
4\end{array}$ & RBFFS_SA & $\begin{array}{c}\text { Ratio } \\
\text { RBFFS_SA } \\
\text { to LB }\end{array}$ & $\begin{array}{l}\text { Ratio Best } \\
\text { SH to } \\
\text { RBFFS_SA }\end{array}$ & $\begin{array}{c}\text { Ratio } \\
\text { RBFFS to } \\
\text { RBFFS_SA }\end{array}$ \\
\hline Exp 1 & 236 & 1.0776 & 1.1398 & 1.1017 & Exp 1 & 228 & 1.0654 & 1.1228 & 1.1579 \\
\hline Exp 2 & 231 & 1.0548 & 1.1645 & 1.1255 & Exp 2 & 229 & 1.0701 & 1.1179 & 1.1528 \\
\hline Exp 3 & 231 & 1.0548 & 1.1645 & 1.1255 & Exp 3 & 224 & 1.0467 & 1.1429 & 1.1786 \\
\hline Exp 4 & 232 & 1.0594 & 1.1595 & 1.1207 & Exp 4 & 232 & 1.0841 & 1.1034 & 1.1379 \\
\hline Exp 5 & 228 & 1.0411 & 1.1798 & 1.1404 & Exp 5 & 231 & 1.0794 & 1.1082 & 1.1429 \\
\hline Exp 6 & 229 & 1.0457 & 1.1747 & 1.1354 & Exp 6 & 230 & 1.0748 & 1.1130 & 1.1478 \\
\hline Exp 7 & 236 & 1.0776 & 1.1398 & 1.1017 & Exp 7 & 230 & 1.0748 & 1.1130 & 1.1478 \\
\hline Exp 8 & 232 & 1.0594 & 1.1595 & 1.1207 & Exp 8 & 228 & 1.0654 & 1.1228 & 1.1579 \\
\hline Exp 9 & 234 & 1.0685 & 1.1496 & 1.1111 & Exp 9 & 226 & 1.0561 & 1.1327 & 1.1681 \\
\hline Exp 10 & 234 & 1.0685 & 1.1496 & 1.1111 & Exp 10 & 232 & 1.0841 & 1.1034 & 1.1379 \\
\hline
\end{tabular}




\begin{tabular}{|c|c|c|c|c|c|c|c|c|c|}
\hline \multicolumn{2}{|c|}{ Data set 5} & Ratio to LB & \multirow{2}{*}{\multicolumn{2}{|c|}{$\begin{array}{c}\text { Stage Configuration } \\
3-1-2 \\
\end{array}$}} & \multicolumn{2}{|l|}{ Data set 6} & Ratio to LB & \multirow{2}{*}{\multicolumn{2}{|c|}{$\begin{array}{c}\text { Stage Configuration } \\
3-1-2 \\
\end{array}$}} \\
\hline LB & 246 & & & & LB & 231 & & & \\
\hline $\mathrm{SH} 1$ & 305 & 1.2398 & \multicolumn{2}{|c|}{ Buffers size $=3$} & $\mathrm{SH} 1$ & 291 & 1.2597 & \multicolumn{2}{|c|}{ Buffers size $=3$} \\
\hline $\mathrm{SH} 2$ & 303 & 1.2317 & & & $\mathrm{SH} 2$ & 293 & 1.2684 & & \\
\hline RBFFS & 298 & 1.2114 & & & RBFFS & 284 & 1.2294 & & \\
\hline $\begin{array}{l}\text { Data set } \\
5\end{array}$ & RBFFS_SA & $\begin{array}{l}\text { Ratio } \\
\text { RBFFS_SA } \\
\text { to LB }\end{array}$ & $\begin{array}{c}\text { Ratio Best } \\
\text { SH to } \\
\text { RBFFS_SA }\end{array}$ & $\begin{array}{c}\text { Ratio } \\
\text { RBFFS to } \\
\text { RBFFS_SA }\end{array}$ & Data set 6 & RBFFS_SA & $\begin{array}{l}\text { Ratio } \\
\text { RBFFS_SA } \\
\text { to LB }\end{array}$ & $\begin{array}{l}\text { Ratio Best } \\
\text { SH to } \\
\text { RBFFS_SA }\end{array}$ & $\begin{array}{c}\text { Ratio } \\
\text { RBFFS to } \\
\text { RBFFS_SA }\end{array}$ \\
\hline Exp 1 & 272 & 1.1057 & 1.1140 & 1.0956 & Exp 1 & 246 & 1.0649 & 1.1829 & 1.1545 \\
\hline Exp 2 & 269 & 1.0935 & 1.1264 & 1.1078 & Exp 2 & 245 & 1.0606 & 1.1878 & 1.1592 \\
\hline Exp 3 & 267 & 1.0854 & 1.1348 & 1.1161 & Exp 3 & 246 & 1.0649 & 1.1829 & 1.1545 \\
\hline Exp 4 & 270 & 1.0976 & 1.1222 & 1.1037 & Exp 4 & 254 & 1.0996 & 1.1457 & 1.1181 \\
\hline Exp 5 & 267 & 1.0854 & 1.1348 & 1.1161 & Exp 5 & 249 & 1.0779 & 1.1687 & 1.1406 \\
\hline Exp 6 & 271 & 1.1016 & 1.1181 & 1.0996 & Exp 6 & 255 & 1.1039 & 1.1412 & 1.1137 \\
\hline Exp 7 & 266 & 1.0813 & 1.1391 & 1.1203 & Exp 7 & 252 & 1.0909 & 1.1548 & 1.1270 \\
\hline Exp 8 & 270 & 1.0976 & 1.1222 & 1.1037 & Exp 8 & 252 & 1.0909 & 1.1548 & 1.1270 \\
\hline Exp 9 & 272 & 1.1057 & 1.1140 & 1.0956 & Exp 9 & 247 & 1.0693 & 1.1781 & 1.1498 \\
\hline Exp 10 & 271 & 1.1016 & 1.1181 & 1.0996 & Exp 10 & 252 & 1.0909 & 1.1548 & 1.1270 \\
\hline
\end{tabular}




\begin{tabular}{|c|c|c|c|c|c|c|c|c|c|}
\hline \multicolumn{2}{|c|}{ Data set 7} & \multirow[t]{2}{*}{ Ratio to LB } & \multirow{2}{*}{\multicolumn{2}{|c|}{$\begin{array}{c}\text { Stage Configuration } \\
3-1-2 \\
\end{array}$}} & \multicolumn{2}{|c|}{ Data set 8} & \multirow[t]{2}{*}{ Ratio to LB } & \multirow{2}{*}{\multicolumn{2}{|c|}{$\begin{array}{c}\text { Stage Configuration } \\
3-1-2 \\
\end{array}$}} \\
\hline LB & 224 & & & & LB & 230 & & & \\
\hline $\mathrm{SH} 1$ & 280 & 1.2500 & \multicolumn{2}{|c|}{ Buffers size $=3$} & $\mathrm{SH} 1$ & 299 & 1.3000 & \multicolumn{2}{|c|}{ Buffers size $=3$} \\
\hline $\mathrm{SH} 2$ & 274 & 1.2232 & & & $\mathrm{SH} 2$ & 290 & 1.2609 & & \\
\hline RBFFS & 268 & 1.1964 & & & RBFFS & 303 & 1.3174 & & \\
\hline $\begin{array}{l}\text { Data } \\
\text { set } 7\end{array}$ & RBFFS_SA & $\begin{array}{c}\text { Ratio } \\
\text { RBFFS_SA } \\
\text { to LB }\end{array}$ & $\begin{array}{l}\text { Ratio Best } \\
\text { SH to } \\
\text { RBFFS_SA }\end{array}$ & $\begin{array}{c}\text { Ratio } \\
\text { RBFFS to } \\
\text { RBFFS_SA }\end{array}$ & $\begin{array}{l}\text { Data set } \\
8\end{array}$ & RBFFS_SA & $\begin{array}{c}\text { Ratio } \\
\text { RBFFS_SA } \\
\text { to LB }\end{array}$ & $\begin{array}{l}\text { Ratio Best } \\
\text { SH to } \\
\text { RBFFS_SA }\end{array}$ & $\begin{array}{c}\text { Ratio } \\
\text { RBFFS to } \\
\text { RBFFS_SA }\end{array}$ \\
\hline Exp 1 & 239 & 1.0670 & 1.1464 & 1.1213 & Exp 1 & 256 & 1.1130 & 1.1328 & 1.1836 \\
\hline Exp 2 & 241 & 1.0759 & 1.1369 & 1.1120 & Exp 2 & 254 & 1.1043 & 1.1417 & 1.1929 \\
\hline Exp 3 & 236 & 1.0536 & 1.1610 & 1.1356 & Exp 3 & 253 & 1.1000 & 1.1462 & 1.1976 \\
\hline Exp 4 & 237 & 1.0580 & 1.1561 & 1.1308 & Exp 4 & 259 & 1.1261 & 1.1197 & 1.1699 \\
\hline Exp 5 & 236 & 1.0536 & 1.1610 & 1.1356 & Exp 5 & 258 & 1.1217 & 1.1240 & 1.1744 \\
\hline Exp 6 & 240 & 1.0714 & 1.1417 & 1.1167 & Exp 6 & 256 & 1.1130 & 1.1328 & 1.1836 \\
\hline Exp 7 & 240 & 1.0714 & 1.1417 & 1.1167 & Exp 7 & 259 & 1.1261 & 1.1197 & 1.1699 \\
\hline Exp 8 & 241 & 1.0759 & 1.1369 & 1.1120 & Exp 8 & 257 & 1.1174 & 1.1284 & 1.1790 \\
\hline Exp 9 & 239 & 1.0670 & 1.1464 & 1.1213 & Exp 9 & 256 & 1.1130 & 1.1328 & 1.1836 \\
\hline Exp 10 & 237 & 1.0580 & 1.1561 & 1.1308 & Exp 10 & 257 & 1.1174 & 1.1284 & 1.1790 \\
\hline
\end{tabular}




\begin{tabular}{|c|c|c|c|c|c|c|c|c|c|}
\hline \multicolumn{2}{|c|}{ Data set 9} & \multirow[t]{2}{*}{ Ratio to LB } & \multirow{2}{*}{\multicolumn{2}{|c|}{$\begin{array}{c}\text { Stage Configuration } \\
3-1-2 \\
\end{array}$}} & \multicolumn{2}{|c|}{ Data set 10} & \multirow[t]{2}{*}{ Ratio to LB } & \multirow{2}{*}{\multicolumn{2}{|c|}{$\begin{array}{c}\text { Stage Configuration } \\
3-1-2 \\
\end{array}$}} \\
\hline LB & 258 & & & & LB & 199 & & & \\
\hline $\mathrm{SH} 1$ & 322 & 1.2481 & \multicolumn{2}{|c|}{ Buffers size $=3$} & $\mathrm{SH} 1$ & 253 & 1.2714 & \multicolumn{2}{|c|}{ Buffers size $=3$} \\
\hline $\mathrm{SH} 2$ & 324 & 1.2558 & & & $\mathrm{SH} 2$ & 255 & 1.2814 & & \\
\hline RBFFS & 301 & 1.1667 & & & RBFFS & 257 & 1.2915 & & \\
\hline $\begin{array}{l}\text { Data set } \\
9\end{array}$ & RBFFS_SA & $\begin{array}{l}\text { Ratio } \\
\text { RBFFS_SA } \\
\text { to LB }\end{array}$ & $\begin{array}{c}\text { Ratio Best } \\
\text { SH to } \\
\text { RBFFS_SA }\end{array}$ & $\begin{array}{c}\text { Ratio } \\
\text { RBFFS to } \\
\text { RBFFS_SA }\end{array}$ & $\begin{array}{l}\text { Data set } \\
10\end{array}$ & RBFFS_SA & $\begin{array}{l}\text { Ratio } \\
\text { RBFFS_SA } \\
\text { to LB }\end{array}$ & $\begin{array}{l}\text { Ratio Best } \\
\text { SH to } \\
\text { RBFFS_SA }\end{array}$ & $\begin{array}{c}\text { Ratio } \\
\text { RBFFS to } \\
\text { RBFFS_SA }\end{array}$ \\
\hline Exp 1 & 275 & 1.0659 & 1.1709 & 1.0945 & Exp 1 & 225 & 1.1307 & 1.1244 & 1.1422 \\
\hline Exp 2 & 280 & 1.0853 & 1.1500 & 1.0750 & Exp 2 & 220 & 1.1055 & 1.1500 & 1.1682 \\
\hline Exp 3 & 278 & 1.0775 & 1.1583 & 1.0827 & Exp 3 & 222 & 1.1156 & 1.1396 & 1.1577 \\
\hline Exp 4 & 277 & 1.0736 & 1.1625 & 1.0866 & Exp 4 & 226 & 1.1357 & 1.1195 & 1.1372 \\
\hline Exp 5 & 276 & 1.0698 & 1.1667 & 1.0906 & Exp 5 & 223 & 1.1206 & 1.1345 & 1.1525 \\
\hline Exp 6 & 282 & 1.0930 & 1.1418 & 1.0674 & Exp 6 & 224 & 1.1256 & 1.1295 & 1.1473 \\
\hline Exp 7 & 277 & 1.0736 & 1.1625 & 1.0866 & Exp 7 & 223 & 1.1206 & 1.1345 & 1.1525 \\
\hline Exp 8 & 276 & 1.0698 & 1.1667 & 1.0906 & Exp 8 & 221 & 1.1106 & 1.1448 & 1.1629 \\
\hline Exp 9 & 276 & 1.0698 & 1.1667 & 1.0906 & Exp 9 & 223 & 1.1206 & 1.1345 & 1.1525 \\
\hline Exp 10 & 277 & 1.0736 & 1.1625 & 1.0866 & Exp 10 & 222 & 1.1156 & 1.1396 & 1.1577 \\
\hline
\end{tabular}




\begin{tabular}{|c|c|c|c|c|c|c|c|c|c|}
\hline \multicolumn{2}{|l|}{ Data set 1} & \multirow[t]{2}{*}{ Ratio to LB } & \multirow{2}{*}{\multicolumn{2}{|c|}{$\begin{array}{c}\text { Stage Configuration } \\
3-1-2\end{array}$}} & \multicolumn{2}{|l|}{ Data set 2} & \multirow[t]{2}{*}{$\begin{array}{c}\text { Ratio to } \\
\text { LB }\end{array}$} & \multirow{2}{*}{\multicolumn{2}{|c|}{$\begin{array}{c}\text { Stage Configuration } \\
3-1-2\end{array}$}} \\
\hline LB & 205 & & & & LB & 228 & & & \\
\hline $\mathrm{SH} 1$ & 275 & 1.3415 & \multicolumn{2}{|c|}{ Buffers size $=3$} & $\mathrm{SH} 1$ & 285 & 1.2500 & \multicolumn{2}{|c|}{ Buffers size $=3$} \\
\hline $\mathrm{SH} 2$ & 265 & 1.2927 & & & $\mathrm{SH} 2$ & 281 & 1.2325 & & \\
\hline PBFFS & 245 & 1.1951 & & & PBFFS & 266 & 1.1667 & & \\
\hline Data set 1 & PBFFS_SA & $\begin{array}{l}\text { Ratio } \\
\text { PBFFS_SA } \\
\text { to LB }\end{array}$ & $\begin{array}{c}\text { Ratio Best } \\
\text { SH to } \\
\text { PBFFS_SA }\end{array}$ & $\begin{array}{c}\text { Ratio } \\
\text { PBFFS to } \\
\text { PBFFS_SA }\end{array}$ & Data set 2 & PBFFS_SA & $\begin{array}{l}\text { Ratio } \\
\text { PBFFS_SA } \\
\text { to LB }\end{array}$ & $\begin{array}{c}\text { Ratio Best } \\
\text { SH to } \\
\text { PBFFS_SA }\end{array}$ & $\begin{array}{c}\text { Ratio } \\
\text { PBFFS to } \\
\text { PBFFS_SA }\end{array}$ \\
\hline Exp 1 & 237 & 1.1561 & 1.1181 & 1.0338 & Exp 1 & 263 & 1.1535 & 1.0684 & 1.0114 \\
\hline Exp 2 & 234 & 1.1415 & 1.1325 & 1.0470 & Exp 2 & 255 & 1.1184 & 1.1020 & 1.0431 \\
\hline Exp 3 & 234 & 1.1415 & 1.1325 & 1.0470 & Exp 3 & 261 & 1.1447 & 1.0766 & 1.0192 \\
\hline Exp 4 & 228 & 1.1122 & 1.1623 & 1.0746 & Exp 4 & 257 & 1.1272 & 1.0934 & 1.0350 \\
\hline Exp 5 & 234 & 1.1415 & 1.1325 & 1.0470 & Exp 5 & 254 & 1.1140 & 1.1063 & 1.0472 \\
\hline Exp 6 & 236 & 1.1512 & 1.1229 & 1.0381 & Exp 6 & 255 & 1.1184 & 1.1020 & 1.0431 \\
\hline $\operatorname{Exp} 7$ & 232 & 1.1317 & 1.1422 & 1.0560 & Exp 7 & 261 & 1.1447 & 1.0766 & 1.0192 \\
\hline Exp 9 & 238 & 1.1610 & 1.1134 & 1.0294 & Exp 9 & 257 & 1.1272 & 1.0934 & 1.0350 \\
\hline Exp 10 & 234 & 1.1415 & 1.1325 & 1.0470 & Exp 10 & 258 & 1.1316 & 1.0891 & 1.0310 \\
\hline
\end{tabular}




\begin{tabular}{|c|c|c|c|c|c|c|c|c|c|}
\hline \multicolumn{2}{|c|}{ Data set 3} & \multirow[t]{2}{*}{$\begin{array}{c}\text { Ratio to } \\
\text { LB }\end{array}$} & \multirow{2}{*}{\multicolumn{2}{|c|}{$\begin{array}{c}\text { Stage Configuration } \\
3-1-2\end{array}$}} & \multicolumn{2}{|c|}{ Data set 4} & \multirow[t]{2}{*}{$\begin{array}{c}\text { Ratio to } \\
\text { LB }\end{array}$} & \multirow{2}{*}{\multicolumn{2}{|c|}{$\begin{array}{c}\text { Stage Configuration } \\
3-1-2 \\
\end{array}$}} \\
\hline LB & 219 & & & & LB & 214 & & & \\
\hline $\mathrm{SH} 1$ & 269 & 1.2283 & \multicolumn{2}{|c|}{ Buffers size $=3$} & $\mathrm{SH} 1$ & 256 & 1.1963 & \multicolumn{2}{|c|}{ Buffers size $=3$} \\
\hline $\mathrm{SH} 2$ & 273 & 1.2466 & & & $\mathrm{SH} 2$ & 268 & 1.2523 & & \\
\hline PBFFS & 255 & 1.1644 & & & PBFFS & 254 & 1.1869 & & \\
\hline $\begin{array}{l}\text { Data set } \\
3\end{array}$ & PBFFS_SA & $\begin{array}{l}\text { Ratio } \\
\text { PBFFS_SA } \\
\text { to LB }\end{array}$ & $\begin{array}{c}\text { Ratio Best } \\
\text { SH to } \\
\text { PBFFS_SA }\end{array}$ & $\begin{array}{l}\text { Ratio } \\
\text { PBFFS to } \\
\text { PBFFS_SA }\end{array}$ & $\begin{array}{l}\text { Data set } \\
4\end{array}$ & PBFFS_SA & $\begin{array}{c}\text { Ratio } \\
\text { PBFFS_SA } \\
\text { to LB }\end{array}$ & $\begin{array}{c}\text { Ratio Best } \\
\text { SH to } \\
\text { PBFFS_SA }\end{array}$ & $\begin{array}{l}\text { Ratio } \\
\text { PBFFS to } \\
\text { PBFFS_SA }\end{array}$ \\
\hline Exp 1 & 238 & 1.0868 & 1.1303 & 1.0714 & Exp 1 & 240 & 1.1215 & 1.0667 & 1.0583 \\
\hline Exp 2 & 241 & 1.1005 & 1.1162 & 1.0581 & Exp 2 & 241 & 1.1262 & 1.0622 & 1.0539 \\
\hline Exp 3 & 249 & 1.1370 & 1.0803 & 1.0241 & Exp 3 & 240 & 1.1215 & 1.0667 & 1.0583 \\
\hline Exp 4 & 246 & 1.1233 & 1.0935 & 1.0366 & Exp 4 & 242 & 1.1308 & 1.0579 & 1.0496 \\
\hline Exp 5 & 244 & 1.1142 & 1.1025 & 1.0451 & Exp 5 & 242 & 1.1308 & 1.0579 & 1.0496 \\
\hline Exp 6 & 240 & 1.0959 & 1.1208 & 1.0625 & Exp 6 & 244 & 1.1402 & 1.0492 & 1.0410 \\
\hline Exp 7 & 237 & 1.0822 & 1.1350 & 1.0759 & Exp 7 & 239 & 1.1168 & 1.0711 & 1.0628 \\
\hline Exp 8 & 242 & 1.1050 & 1.1116 & 1.0537 & Exp 8 & 242 & 1.1308 & 1.0579 & 1.0496 \\
\hline Exp 9 & 246 & 1.1233 & 1.0935 & 1.0366 & Exp 9 & 241 & 1.1262 & 1.0622 & 1.0539 \\
\hline Exp 10 & 242 & 1.1050 & 1.1116 & 1.0537 & Exp 10 & 245 & 1.1449 & 1.0449 & 1.0367 \\
\hline
\end{tabular}




\begin{tabular}{|c|c|c|c|c|c|c|c|c|c|}
\hline \multicolumn{2}{|c|}{ Data set 5} & \multirow[t]{2}{*}{$\begin{array}{c}\text { Ratio to } \\
\text { LB }\end{array}$} & \multirow{2}{*}{\multicolumn{2}{|c|}{$\begin{array}{c}\text { Stage Configuration } \\
3-1-2\end{array}$}} & \multicolumn{2}{|c|}{ Data set 6} & \multirow[t]{2}{*}{$\begin{array}{c}\text { Ratio to } \\
\text { LB }\end{array}$} & \multirow{2}{*}{\multicolumn{2}{|c|}{$\begin{array}{c}\text { Stage Configuration } \\
3-1-2 \\
\end{array}$}} \\
\hline LB & 246 & & & & LB & 231 & & & \\
\hline $\mathrm{SH} 1$ & 305 & 1.2398 & \multicolumn{2}{|c|}{ Buffers size $=3$} & $\mathrm{SH} 1$ & 291 & 1.2597 & \multicolumn{2}{|c|}{ Buffers size $=3$} \\
\hline $\mathrm{SH} 2$ & 303 & 1.2317 & & & $\mathrm{SH} 2$ & 293 & 1.2684 & & \\
\hline PBFFS & 292 & 1.1870 & & & PBFFS & 279 & 1.2078 & & \\
\hline $\begin{array}{l}\text { Data set } \\
5\end{array}$ & PBFFS_SA & $\begin{array}{c}\text { Ratio } \\
\text { PBFFS_SA } \\
\text { to LB }\end{array}$ & $\begin{array}{c}\text { Ratio Best } \\
\text { SH to } \\
\text { PBFFS_SA }\end{array}$ & $\begin{array}{l}\text { Ratio } \\
\text { PBFFS to } \\
\text { PBFFS_SA }\end{array}$ & $\begin{array}{l}\text { Data } \\
\text { set } 6\end{array}$ & PBFFS_SA & $\begin{array}{l}\text { Ratio } \\
\text { PBFFS_SA } \\
\text { to LB }\end{array}$ & $\begin{array}{c}\text { Ratio Best } \\
\text { SH to } \\
\text { PBFFS_SA }\end{array}$ & $\begin{array}{c}\text { Ratio } \\
\text { PBFFS to } \\
\text { PBFFS_SA }\end{array}$ \\
\hline Exp 1 & 283 & 1.1504 & 1.0707 & 1.0318 & Exp 1 & 267 & 1.1558 & 1.0899 & 1.0449 \\
\hline Exp 2 & 281 & 1.1423 & 1.0783 & 1.0391 & Exp 2 & 261 & 1.1299 & 1.1149 & 1.0690 \\
\hline Exp 3 & 283 & 1.1504 & 1.0707 & 1.0318 & Exp 3 & 262 & 1.1342 & 1.1107 & 1.0649 \\
\hline Exp 4 & 284 & 1.1545 & 1.0669 & 1.0282 & Exp 4 & 266 & 1.1515 & 1.0940 & 1.0489 \\
\hline Exp 5 & 282 & 1.1463 & 1.0745 & 1.0355 & Exp 5 & 266 & 1.1515 & 1.0940 & 1.0489 \\
\hline Exp 6 & 285 & 1.1585 & 1.0632 & 1.0246 & Exp 6 & 261 & 1.1299 & 1.1149 & 1.0690 \\
\hline Exp 7 & 280 & 1.1382 & 1.0821 & 1.0429 & Exp 7 & 266 & 1.1515 & 1.0940 & 1.0489 \\
\hline Exp 8 & 280 & 1.1382 & 1.0821 & 1.0429 & Exp 8 & 263 & 1.1385 & 1.1065 & 1.0608 \\
\hline Exp 9 & 281 & 1.1423 & 1.0783 & 1.0391 & Exp 9 & 266 & 1.1515 & 1.0940 & 1.0489 \\
\hline Exp 10 & 285 & 1.1585 & 1.0632 & 1.0246 & Exp 10 & 267 & 1.1558 & 1.0899 & 1.0449 \\
\hline
\end{tabular}




\begin{tabular}{|c|c|c|c|c|c|c|c|c|c|}
\hline \multicolumn{2}{|c|}{ Data set 7} & \multirow[t]{2}{*}{$\begin{array}{l}\text { Ratio to } \\
\text { LB }\end{array}$} & \multirow{2}{*}{\multicolumn{2}{|c|}{$\begin{array}{c}\text { Stage Configuration } \\
3-1-2\end{array}$}} & \multicolumn{2}{|c|}{ Data set 8} & \multirow[t]{2}{*}{ Ratio to LB } & \multirow{2}{*}{\multicolumn{2}{|c|}{$\begin{array}{c}\text { Stage Configuration } \\
3-1-2\end{array}$}} \\
\hline LB & 224 & & & & LB & 230 & & & \\
\hline $\mathrm{SH} 1$ & 280 & 1.2500 & \multicolumn{2}{|c|}{ Buffers size $=3$} & $\mathrm{SH} 1$ & 299 & 1.3000 & \multicolumn{2}{|c|}{ Buffers size $=3$} \\
\hline $\mathrm{SH} 2$ & 274 & 1.2232 & & & $\mathrm{SH} 2$ & 290 & 1.2609 & & \\
\hline PBFFS & 263 & 1.1741 & & & PBFFS & 280 & 1.2174 & & \\
\hline $\begin{array}{l}\text { Data } \\
\text { set } 7\end{array}$ & PBFFS_SA & $\begin{array}{l}\text { Ratio } \\
\text { PBFFS_SA } \\
\text { to LB }\end{array}$ & $\begin{array}{c}\text { Ratio Best } \\
\text { SH to } \\
\text { PBFFS_SA }\end{array}$ & $\begin{array}{c}\text { Ratio } \\
\text { PBFFS to } \\
\text { PBFFS_SA }\end{array}$ & $\begin{array}{l}\text { Data } \\
\text { set } 8\end{array}$ & PBFFS_SA & $\begin{array}{l}\text { Ratio } \\
\text { PBFFS_SA } \\
\text { to LB }\end{array}$ & $\begin{array}{c}\text { Ratio Best } \\
\text { SH to } \\
\text { PBFFS_SA }\end{array}$ & $\begin{array}{c}\text { Ratio PBFFS } \\
\text { to } \\
\text { PBFFS_SA }\end{array}$ \\
\hline Exp 1 & 252 & 1.1250 & 1.0873 & 1.0437 & Exp 1 & 270 & 1.1739 & $1.074 \overline{1}$ & 1.0370 \\
\hline Exp 2 & 246 & 1.0982 & 1.1138 & 1.0691 & Exp 2 & 268 & 1.1652 & 1.0821 & 1.0448 \\
\hline Exp 3 & 256 & 1.1429 & 1.0703 & 1.0273 & Exp 3 & 270 & 1.1739 & 1.0741 & 1.0370 \\
\hline Exp 4 & 253 & 1.1295 & 1.0830 & 1.0395 & Exp 4 & 269 & 1.1696 & 1.0781 & 1.0409 \\
\hline Exp 5 & 253 & 1.1295 & 1.0830 & 1.0395 & Exp 5 & 272 & 1.1826 & 1.0662 & 1.0294 \\
\hline Exp 6 & 249 & 1.1116 & 1.1004 & 1.0562 & Exp 6 & 272 & 1.1826 & 1.0662 & 1.0294 \\
\hline Exp 7 & 250 & 1.1161 & 1.0960 & 1.0520 & Exp 7 & 270 & 1.1739 & 1.0741 & 1.0370 \\
\hline Exp 8 & 254 & 1.1339 & 1.0787 & 1.0354 & Exp 8 & 268 & 1.1652 & 1.0821 & 1.0448 \\
\hline Exp 9 & 251 & 1.1205 & 1.0916 & 1.0478 & Exp 9 & 273 & 1.1870 & 1.0623 & 1.0256 \\
\hline Exp 10 & 252 & 1.1250 & 1.0873 & 1.0437 & Exp 10 & 269 & 1.1696 & 1.0781 & 1.0409 \\
\hline
\end{tabular}




\begin{tabular}{|c|c|c|c|c|c|c|c|c|c|}
\hline \multicolumn{2}{|c|}{ Data set 9} & \multirow[t]{2}{*}{ Ratio to LB } & \multirow{2}{*}{\multicolumn{2}{|c|}{$\begin{array}{c}\text { Stage Configuration } \\
3-1-2\end{array}$}} & \multicolumn{2}{|c|}{ Data set 10} & \multirow[t]{2}{*}{$\begin{array}{c}\text { Ratio to } \\
\text { LB }\end{array}$} & \multirow{2}{*}{\multicolumn{2}{|c|}{$\begin{array}{c}\text { Stage Configuration } \\
3-1-2 \\
\end{array}$}} \\
\hline LB & 258 & & & & LB & 199 & & & \\
\hline $\mathrm{SH} 1$ & 322 & 1.2481 & \multicolumn{2}{|c|}{ Buffers size $=3$} & $\mathrm{SH} 1$ & 253 & 1.2714 & \multicolumn{2}{|c|}{ Buffers size $=3$} \\
\hline $\mathrm{SH} 2$ & 324 & 1.2558 & & & $\mathrm{SH} 2$ & 255 & 1.2814 & & \\
\hline PBFFS & 303 & 1.1744 & & & PBFFS & 241 & 1.2111 & & \\
\hline $\begin{array}{l}\text { Data } \\
\text { set } 9\end{array}$ & PBFFS_SA & $\begin{array}{l}\text { Ratio } \\
\text { PBFFS_SA } \\
\text { to LB }\end{array}$ & $\begin{array}{c}\text { Ratio Best } \\
\text { SH to } \\
\text { PBFFS_SA }\end{array}$ & $\begin{array}{l}\text { Ratio } \\
\text { PBFFS to } \\
\text { PBFFS_SA }\end{array}$ & $\begin{array}{l}\text { Data } \\
\text { set } 10\end{array}$ & PBFFS_SA & $\begin{array}{l}\text { Ratio } \\
\text { PBFFS_SA } \\
\text { to LB }\end{array}$ & $\begin{array}{c}\text { Ratio Best } \\
\text { SH to } \\
\text { PBFFS_SA }\end{array}$ & $\begin{array}{l}\text { Ratio } \\
\text { PBFFS to } \\
\text { PBFFS_SA }\end{array}$ \\
\hline Exp 1 & 287 & 1.1124 & 1.1220 & 1.0557 & Exp 1 & 230 & 1.1558 & 1.1000 & 1.0478 \\
\hline Exp 2 & 293 & 1.1357 & 1.0990 & 1.0341 & Exp 2 & 229 & 1.1508 & 1.1048 & 1.0524 \\
\hline Exp 3 & 295 & 1.1434 & 1.0915 & 1.0271 & Exp 3 & 230 & 1.1558 & 1.1000 & 1.0478 \\
\hline Exp 4 & 293 & 1.1357 & 1.0990 & 1.0341 & Exp 4 & 231 & 1.1608 & 1.0952 & 1.0433 \\
\hline Exp 5 & 299 & 1.1589 & 1.0769 & 1.0134 & Exp 5 & 231 & 1.1608 & 1.0952 & 1.0433 \\
\hline Exp 6 & 291 & 1.1279 & 1.1065 & 1.0412 & Exp 6 & 227 & 1.1407 & 1.1145 & 1.0617 \\
\hline Exp 7 & 293 & 1.1357 & 1.0990 & 1.0341 & Exp 7 & 229 & 1.1508 & 1.1048 & 1.0524 \\
\hline Exp 8 & 293 & 1.1357 & 1.0990 & 1.0341 & Exp 8 & 233 & 1.1709 & 1.0858 & 1.0343 \\
\hline Exp 9 & 289 & 1.1202 & 1.1142 & 1.0484 & Exp 9 & 234 & 1.1759 & 1.0812 & 1.0299 \\
\hline Exp 10 & 296 & 1.1473 & 1.0878 & 1.0236 & Exp 10 & 232 & 1.1658 & 1.0905 & 1.0388 \\
\hline
\end{tabular}




\begin{tabular}{|c|c|c|c|c|c|c|c|c|c|}
\hline \multicolumn{2}{|c|}{ Data set 1} & Ratio to LB & \multirow{2}{*}{\multicolumn{2}{|c|}{$\begin{array}{c}\text { Stage Configuration } \\
2-1-2-1 \\
\end{array}$}} & \multicolumn{2}{|c|}{ Data set 2} & Ratio to LB & \multirow{2}{*}{\multicolumn{2}{|c|}{$\begin{array}{c}\text { Stage Configuration } \\
2-1-2-1 \\
\end{array}$}} \\
\hline LB & 206 & & & & LB & 237 & & & \\
\hline $\mathrm{SH} 1$ & 280 & 1.3592 & \multicolumn{2}{|c|}{ Buffers size $=3$} & $\mathrm{SH} 1$ & 310 & 1.3080 & \multicolumn{2}{|c|}{ Buffers size $=3$} \\
\hline $\mathrm{SH} 2$ & 277 & 1.3447 & & & $\mathrm{SH} 2$ & 298 & 1.2574 & & \\
\hline RBFFS & 268 & 1.3010 & & & RBFFS & 284 & 1.1983 & & \\
\hline $\begin{array}{l}\text { Data set } \\
1\end{array}$ & RBFFS_SA & $\begin{array}{l}\text { Ratio } \\
\text { RBFFS_SA } \\
\text { to LB }\end{array}$ & $\begin{array}{l}\text { Ratio Best } \\
\text { SH to } \\
\text { RBFFS_SA }\end{array}$ & $\begin{array}{c}\text { Ratio } \\
\text { RBFFS to } \\
\text { RBFFS_SA }\end{array}$ & $\begin{array}{l}\text { Data set } \\
2\end{array}$ & RBFFS_SA & $\begin{array}{l}\text { Ratio } \\
\text { RBFFS_SA } \\
\text { to LB }\end{array}$ & $\begin{array}{c}\text { Ratio Best } \\
\text { SH to } \\
\text { RBFFS_SA }\end{array}$ & $\begin{array}{c}\text { Ratio } \\
\text { RBFFS to } \\
\text { RBFFS_SA }\end{array}$ \\
\hline Exp 1 & 240 & 1.1650 & 1.1542 & 1.1167 & Exp 1 & 258 & 1.0886 & 1.1550 & 1.1008 \\
\hline Exp 2 & 238 & 1.1553 & 1.1639 & 1.1261 & Exp 2 & 266 & 1.1224 & 1.1203 & 1.0677 \\
\hline Exp 3 & 239 & 1.1602 & 1.1590 & 1.1213 & Exp 3 & 264 & 1.1139 & 1.1288 & 1.0758 \\
\hline Exp 4 & 239 & 1.1602 & 1.1590 & 1.1213 & Exp 4 & 263 & 1.1097 & 1.1331 & 1.0798 \\
\hline Exp 5 & 239 & 1.1602 & 1.1590 & 1.1213 & Exp 5 & 263 & 1.1097 & 1.1331 & 1.0798 \\
\hline Exp 6 & 240 & 1.1650 & 1.1542 & 1.1167 & Exp 6 & 267 & 1.1266 & 1.1161 & 1.0637 \\
\hline Exp 7 & 235 & 1.1408 & 1.1787 & 1.1404 & Exp 7 & 265 & 1.1181 & 1.1245 & 1.0717 \\
\hline Exp 9 & 239 & 1.1602 & 1.1590 & 1.1213 & Exp 9 & 265 & 1.1181 & 1.1245 & 1.0717 \\
\hline Exp 10 & 240 & 1.1650 & 1.1542 & 1.1167 & Exp 10 & 266 & 1.1224 & 1.1203 & 1.0677 \\
\hline
\end{tabular}




\begin{tabular}{|c|c|c|c|c|c|c|c|c|c|}
\hline \multicolumn{2}{|c|}{ Data set 3} & Ratio to LB & \multirow{2}{*}{\multicolumn{2}{|c|}{$\begin{array}{c}\text { Stage Configuration } \\
2-1-2-1 \\
\end{array}$}} & \multicolumn{2}{|c|}{ Data set 4} & Ratio to LB & \multirow{2}{*}{\multicolumn{2}{|c|}{$\begin{array}{c}\text { Stage Configuration } \\
2-1-2-1 \\
\end{array}$}} \\
\hline LB & 225 & & & & LB & 214 & & & \\
\hline $\mathrm{SH} 1$ & 294 & 1.3067 & \multicolumn{2}{|c|}{ Buffers size $=3$} & $\mathrm{SH} 1$ & 277 & 1.2944 & \multicolumn{2}{|c|}{ Buffers size $=3$} \\
\hline $\mathrm{SH} 2$ & 296 & 1.3156 & & & $\mathrm{SH} 2$ & 278 & 1.2991 & & \\
\hline RBFFS & 289 & 1.2844 & & & RBFFS & 279 & 1.3037 & & \\
\hline $\begin{array}{l}\text { Data set } \\
3\end{array}$ & RBFFS_SA & $\begin{array}{l}\text { Ratio } \\
\text { RBFFS_SA } \\
\text { to LB }\end{array}$ & $\begin{array}{l}\text { Ratio Best } \\
\text { SH to } \\
\text { RBFFS_SA }\end{array}$ & $\begin{array}{c}\text { Ratio } \\
\text { RBFFS to } \\
\text { RBFFS_SA }\end{array}$ & $\begin{array}{l}\text { Data set } \\
4\end{array}$ & RBFFS_SA & $\begin{array}{c}\text { Ratio } \\
\text { RBFFS_SA } \\
\text { to LB }\end{array}$ & $\begin{array}{c}\text { Ratio Best } \\
\text { SH to } \\
\text { RBFFS_SA }\end{array}$ & $\begin{array}{c}\text { Ratio } \\
\text { RBFFS to } \\
\text { RBFFS_SA }\end{array}$ \\
\hline Exp 1 & 261 & 1.1600 & 1.1264 & 1.1073 & Exp 1 & 244 & 1.1402 & 1.1352 & 1.1434 \\
\hline Exp 2 & 251 & 1.1156 & 1.1713 & 1.1514 & Exp 2 & 237 & 1.1075 & 1.1688 & 1.1772 \\
\hline Exp 3 & 257 & 1.1422 & 1.1440 & 1.1245 & Exp 3 & 241 & 1.1262 & 1.1494 & 1.1577 \\
\hline $\operatorname{Exp} 4$ & 256 & 1.1378 & 1.1484 & 1.1289 & Exp 4 & 238 & 1.1121 & 1.1639 & 1.1723 \\
\hline Exp 5 & 255 & 1.1333 & 1.1529 & 1.1333 & Exp 5 & 245 & 1.1449 & 1.1306 & 1.1388 \\
\hline Exp 6 & 261 & 1.1600 & 1.1264 & 1.1073 & Exp 6 & 246 & 1.1495 & 1.1260 & 1.1341 \\
\hline Exp 7 & 254 & 1.1289 & 1.1575 & 1.1378 & Exp 7 & 246 & 1.1495 & 1.1260 & 1.1341 \\
\hline Exp 8 & 251 & 1.1156 & 1.1713 & 1.1514 & Exp 8 & 243 & 1.1355 & 1.1399 & 1.1481 \\
\hline Exp 9 & 256 & 1.1378 & 1.1484 & 1.1289 & Exp 9 & 241 & 1.1262 & 1.1494 & 1.1577 \\
\hline Exp 10 & 256 & 1.1378 & 1.1484 & 1.1289 & Exp 10 & 245 & 1.1449 & 1.1306 & 1.1388 \\
\hline
\end{tabular}




\begin{tabular}{|c|c|c|c|c|c|c|c|c|c|}
\hline \multicolumn{2}{|c|}{ Data set 5} & Ratio to LB & \multirow{2}{*}{\multicolumn{2}{|c|}{$\begin{array}{c}\text { Stage Configuration } \\
2-1-2-1 \\
\end{array}$}} & \multicolumn{2}{|l|}{ Data set 6} & Ratio to LB & \multirow{2}{*}{\multicolumn{2}{|c|}{$\begin{array}{c}\text { Stage Configuration } \\
2-1-2-1 \\
\end{array}$}} \\
\hline LB & 248 & & & & LB & 234 & & & \\
\hline $\mathrm{SH} 1$ & 312 & 1.2581 & \multicolumn{2}{|c|}{ Buffers size $=3$} & $\mathrm{SH} 1$ & 307 & 1.3120 & \multicolumn{2}{|c|}{ Buffers size $=3$} \\
\hline $\mathrm{SH} 2$ & 319 & 1.2863 & & & $\mathrm{SH} 2$ & 294 & 1.2564 & & \\
\hline RBFFS & 315 & 1.2702 & & & RBFFS & 291 & 1.2436 & & \\
\hline $\begin{array}{l}\text { Data set } \\
5\end{array}$ & RBFFS_SA & $\begin{array}{l}\text { Ratio } \\
\text { RBFFS_SA } \\
\text { to LB }\end{array}$ & $\begin{array}{c}\text { Ratio Best } \\
\text { SH to } \\
\text { RBFFS_SA }\end{array}$ & $\begin{array}{c}\text { Ratio } \\
\text { RBFFS to } \\
\text { RBFFS_SA }\end{array}$ & Data set 6 & RBFFS_SA & $\begin{array}{c}\text { Ratio } \\
\text { RBFFS_SA } \\
\text { to LB }\end{array}$ & $\begin{array}{l}\text { Ratio Best } \\
\text { SH to } \\
\text { RBFFS_SA }\end{array}$ & $\begin{array}{c}\text { Ratio } \\
\text { RBFFS to } \\
\text { RBFFS_SA }\end{array}$ \\
\hline Exp 1 & 286 & 1.1532 & 1.0909 & 1.1014 & Exp 1 & 271 & 1.1581 & 1.0849 & 1.0738 \\
\hline Exp 2 & 283 & 1.1411 & 1.1025 & 1.1131 & Exp 2 & 272 & 1.1624 & 1.0809 & 1.0699 \\
\hline Exp 3 & 284 & 1.1452 & 1.0986 & 1.1092 & Exp 3 & 268 & 1.1453 & 1.0970 & 1.0858 \\
\hline Exp 4 & 285 & 1.1492 & 1.0947 & 1.1053 & Exp 4 & 274 & 1.1709 & 1.0730 & 1.0620 \\
\hline Exp 5 & 287 & 1.1573 & 1.0871 & 1.0976 & Exp 5 & 267 & 1.1410 & 1.1011 & 1.0899 \\
\hline Exp 6 & 280 & 1.1290 & 1.1143 & 1.1250 & Exp 6 & 267 & 1.1410 & 1.1011 & 1.0899 \\
\hline Exp 7 & 290 & 1.1694 & 1.0759 & 1.0862 & Exp 7 & 276 & 1.1795 & 1.0652 & 1.0543 \\
\hline Exp 8 & 288 & 1.1613 & 1.0833 & 1.0938 & Exp 8 & 265 & 1.1325 & 1.1094 & 1.0981 \\
\hline Exp 9 & 291 & 1.1734 & 1.0722 & 1.0825 & Exp 9 & 270 & 1.1538 & 1.0889 & 1.0778 \\
\hline Exp 10 & 284 & 1.1452 & 1.0986 & 1.1092 & Exp 10 & 267 & 1.1410 & 1.1011 & 1.0899 \\
\hline
\end{tabular}




\begin{tabular}{|c|c|c|c|c|c|c|c|c|c|}
\hline \multicolumn{2}{|c|}{ Data set 7} & Ratio to LB & \multirow{2}{*}{\multicolumn{2}{|c|}{$\begin{array}{c}\text { Stage Configuration } \\
2-1-2-1 \\
\end{array}$}} & \multicolumn{2}{|c|}{ Data set 8} & Ratio to LB & \multirow{2}{*}{\multicolumn{2}{|c|}{$\begin{array}{c}\text { Stage Configuration } \\
2-1-2-1 \\
\end{array}$}} \\
\hline LB & 224 & & & & LB & 239 & & & \\
\hline $\mathrm{SH} 1$ & 297 & 1.3259 & \multicolumn{2}{|c|}{ Buffers size $=3$} & $\mathrm{SH} 1$ & 304 & 1.2720 & \multicolumn{2}{|c|}{ Buffers size $=3$} \\
\hline $\mathrm{SH} 2$ & 288 & 1.2857 & & & $\mathrm{SH} 2$ & 307 & 1.2845 & & \\
\hline RBFFS & 275 & 1.2277 & & & RBFFS & 295 & 1.2343 & & \\
\hline $\begin{array}{l}\text { Data } \\
\text { set } 7\end{array}$ & RBFFS_SA & $\begin{array}{c}\text { Ratio } \\
\text { RBFFS_SA } \\
\text { to LB }\end{array}$ & $\begin{array}{l}\text { Ratio Best } \\
\text { SH to } \\
\text { RBFFS_SA }\end{array}$ & $\begin{array}{c}\text { Ratio } \\
\text { RBFFS to } \\
\text { RBFFS_SA }\end{array}$ & $\begin{array}{l}\text { Data set } \\
8\end{array}$ & RBFFS_SA & $\begin{array}{c}\text { Ratio } \\
\text { RBFFS_SA } \\
\text { to LB }\end{array}$ & $\begin{array}{c}\text { Ratio Best } \\
\text { SH to } \\
\text { RBFFS_SA }\end{array}$ & $\begin{array}{c}\text { Ratio } \\
\text { RBFFS to } \\
\text { RBFFS_SA }\end{array}$ \\
\hline Exp 1 & 260 & 1.1607 & 1.1077 & 1.0577 & Exp 1 & 275 & 1.1506 & 1.1055 & 1.0727 \\
\hline Exp 2 & 261 & 1.1652 & 1.1034 & 1.0536 & Exp 2 & 273 & 1.1423 & 1.1136 & 1.0806 \\
\hline Exp 3 & 262 & 1.1696 & 1.0992 & 1.0496 & Exp 3 & 272 & 1.1381 & 1.1176 & 1.0846 \\
\hline Exp 4 & 255 & 1.1384 & 1.1294 & 1.0784 & Exp 4 & 274 & 1.1464 & 1.1095 & 1.0766 \\
\hline Exp 5 & 265 & 1.1830 & 1.0868 & 1.0377 & Exp 5 & 272 & 1.1381 & 1.1176 & 1.0846 \\
\hline Exp 6 & 261 & 1.1652 & 1.1034 & 1.0536 & Exp 6 & 276 & 1.1548 & 1.1014 & 1.0688 \\
\hline Exp 7 & 261 & 1.1652 & 1.1034 & 1.0536 & Exp 7 & 275 & 1.1506 & 1.1055 & 1.0727 \\
\hline Exp 8 & 263 & 1.1741 & 1.0951 & 1.0456 & Exp 8 & 271 & 1.1339 & 1.1218 & 1.0886 \\
\hline Exp 9 & 260 & 1.1607 & 1.1077 & 1.0577 & Exp 9 & 274 & 1.1464 & 1.1095 & 1.0766 \\
\hline Exp 10 & 260 & 1.1607 & 1.1077 & 1.0577 & Exp 10 & 270 & 1.1297 & 1.1259 & 1.0926 \\
\hline
\end{tabular}




\begin{tabular}{|c|c|c|c|c|c|c|c|c|c|}
\hline \multicolumn{2}{|c|}{ Data set 9} & \multirow[t]{2}{*}{ Ratio to LB } & \multirow{2}{*}{\multicolumn{2}{|c|}{$\begin{array}{c}\text { Stage Configuration } \\
2-1-2-1 \\
\end{array}$}} & \multicolumn{2}{|c|}{ Data set 10} & \multirow[t]{2}{*}{ Ratio to LB } & \multirow{2}{*}{\multicolumn{2}{|c|}{$\begin{array}{c}\text { Stage Configuration } \\
2-1-2-1\end{array}$}} \\
\hline LB & 262 & & & & LB & 216 & & & \\
\hline $\mathrm{SH} 1$ & 327 & 1.2481 & \multicolumn{2}{|c|}{ Buffers size $=3$} & $\mathrm{SH} 1$ & 276 & 1.2778 & \multicolumn{2}{|c|}{ Buffers size $=3$} \\
\hline $\mathrm{SH} 2$ & 322 & 1.2290 & & & $\mathrm{SH} 2$ & 285 & 1.3194 & & \\
\hline RBFFS & 323 & 1.2328 & & & RBFFS & 275 & 1.2731 & & \\
\hline $\begin{array}{l}\text { Data set } \\
9\end{array}$ & RBFFS_SA & $\begin{array}{l}\text { Ratio } \\
\text { RBFFS_SA } \\
\text { to LB }\end{array}$ & $\begin{array}{l}\text { Ratio Best } \\
\text { SH to } \\
\text { RBFFS_SA }\end{array}$ & $\begin{array}{c}\text { Ratio } \\
\text { RBFFS to } \\
\text { RBFFS_SA }\end{array}$ & $\begin{array}{l}\text { Data set } \\
10\end{array}$ & RBFFS_SA & $\begin{array}{l}\text { Ratio } \\
\text { RBFFS_SA } \\
\text { to LB }\end{array}$ & $\begin{array}{c}\text { Ratio Best } \\
\text { SH to } \\
\text { RBFFS_SA }\end{array}$ & $\begin{array}{c}\text { Ratio } \\
\text { RBFFS to } \\
\text { RBFFS_SA }\end{array}$ \\
\hline Exp 1 & 303 & 1.1565 & 1.0627 & 1.0660 & Exp 1 & 242 & 1.1204 & 1.1405 & 1.1364 \\
\hline Exp 2 & 302 & 1.1527 & 1.0662 & 1.0695 & Exp 2 & 242 & 1.1204 & 1.1405 & 1.1364 \\
\hline Exp 3 & 303 & 1.1565 & 1.0627 & 1.0660 & Exp 3 & 245 & 1.1343 & 1.1265 & 1.1224 \\
\hline Exp 4 & 301 & 1.1489 & 1.0698 & 1.0731 & Exp 4 & 247 & 1.1435 & 1.1174 & 1.1134 \\
\hline Exp 5 & 302 & 1.1527 & 1.0662 & 1.0695 & Exp 5 & 245 & 1.1343 & 1.1265 & 1.1224 \\
\hline Exp 6 & 303 & 1.1565 & 1.0627 & 1.0660 & Exp 6 & 250 & 1.1574 & 1.1040 & 1.1000 \\
\hline Exp 7 & 304 & 1.1603 & 1.0592 & 1.0625 & Exp 7 & 245 & 1.1343 & 1.1265 & 1.1224 \\
\hline Exp 8 & 299 & 1.1412 & 1.0769 & 1.0803 & Exp 8 & 240 & 1.1111 & 1.1500 & 1.1458 \\
\hline Exp 9 & 299 & 1.1412 & 1.0769 & 1.0803 & Exp 9 & 242 & 1.1204 & 1.1405 & 1.1364 \\
\hline Exp 10 & 304 & 1.1603 & 1.0592 & 1.0625 & $\operatorname{Exp} 10$ & 244 & 1.1296 & 1.1311 & 1.1270 \\
\hline
\end{tabular}




\begin{tabular}{|c|c|c|c|c|c|c|c|c|c|}
\hline \multicolumn{2}{|l|}{ Data set 1} & \multirow[t]{2}{*}{ Ratio to LB } & \multirow{2}{*}{\multicolumn{2}{|c|}{$\begin{array}{c}\text { Stage Configuration } \\
2-1-2-1\end{array}$}} & \multicolumn{2}{|l|}{ Data set 2} & \multirow[t]{2}{*}{$\begin{array}{c}\text { Ratio to } \\
\text { LB }\end{array}$} & \multirow{2}{*}{\multicolumn{2}{|c|}{$\begin{array}{c}\text { Stage Configuration } \\
2-1-2-1\end{array}$}} \\
\hline LB & 206 & & & & LB & 237 & & & \\
\hline $\mathrm{SH} 1$ & 280 & 1.3592 & \multicolumn{2}{|c|}{ Buffers size $=3$} & $\mathrm{SH} 1$ & 310 & 1.3080 & \multicolumn{2}{|c|}{ Buffers size $=3$} \\
\hline PBFFS & 256 & 1.2427 & & & PBFFS & 280 & 1.1814 & & \\
\hline Data set 1 & PBFFS_SA & $\begin{array}{l}\text { Ratio } \\
\text { PBFFS_SA } \\
\text { to LB }\end{array}$ & $\begin{array}{c}\text { Ratio Best } \\
\text { SH to } \\
\text { PBFFS_SA }\end{array}$ & $\begin{array}{l}\text { Ratio } \\
\text { PBFFS to } \\
\text { PBFFS_SA }\end{array}$ & Data set 2 & PBFFS_SA & $\begin{array}{l}\text { Ratio } \\
\text { PBFFS_SA } \\
\text { to LB }\end{array}$ & $\begin{array}{c}\text { Ratio Best } \\
\text { SH to } \\
\text { PBFFS_SA }\end{array}$ & $\begin{array}{c}\text { Ratio } \\
\text { PBFFS to } \\
\text { PBFFS_SA }\end{array}$ \\
\hline Exp 1 & 251 & 1.2184 & 1.1036 & 1.0199 & Exp 1 & 270 & 1.1392 & 1.1037 & 1.0370 \\
\hline Exp 2 & 250 & 1.2136 & 1.1080 & 1.0240 & Exp 2 & 274 & 1.1561 & 1.0876 & 1.0219 \\
\hline Exp 3 & 252 & 1.2233 & 1.0992 & 1.0159 & Exp 3 & 273 & 1.1519 & 1.0916 & 1.0256 \\
\hline Exp 4 & 254 & 1.2330 & 1.0906 & 1.0079 & Exp 4 & 273 & 1.1519 & 1.0916 & 1.0256 \\
\hline Exp 5 & 246 & 1.1942 & 1.1260 & 1.0407 & Exp 5 & 267 & 1.1266 & 1.1161 & 1.0487 \\
\hline Exp 6 & 251 & 1.2184 & 1.1036 & 1.0199 & Exp 6 & 272 & 1.1477 & 1.0956 & 1.0294 \\
\hline Exp 7 & 252 & 1.2233 & 1.0992 & 1.0159 & Exp 7 & 276 & 1.1646 & 1.0797 & 1.0145 \\
\hline Exp 9 & 250 & 1.2136 & 1.1080 & 1.0240 & Exp 9 & 274 & 1.1561 & 1.0876 & 1.0219 \\
\hline Exp 10 & 251 & 1.2184 & 1.1036 & 1.0199 & Exp 10 & 269 & 1.1350 & 1.1078 & 1.0409 \\
\hline
\end{tabular}




\begin{tabular}{|c|c|c|c|c|c|c|c|c|c|}
\hline \multicolumn{2}{|c|}{ Data set 3} & \multirow[t]{2}{*}{$\begin{array}{c}\text { Ratio to } \\
\text { LB }\end{array}$} & \multirow{2}{*}{\multicolumn{2}{|c|}{$\begin{array}{c}\text { Stage Configuration } \\
2-1-2-1 \\
\end{array}$}} & \multicolumn{2}{|c|}{ Data set 4} & \multirow[t]{2}{*}{$\begin{array}{c}\text { Ratio to } \\
\text { LB }\end{array}$} & \multirow{2}{*}{\multicolumn{2}{|c|}{$\begin{array}{c}\text { Stage Configuration } \\
2-1-2-1\end{array}$}} \\
\hline LB & 225 & & & & LB & 214 & & & \\
\hline $\mathrm{SH} 1$ & 294 & 1.3067 & \multicolumn{2}{|c|}{ Buffers size $=3$} & $\mathrm{SH} 1$ & 277 & 1.2944 & \multicolumn{2}{|c|}{ Buffers size $=3$} \\
\hline $\mathrm{SH} 2$ & 296 & 1.3156 & & & $\mathrm{SH} 2$ & 278 & 1.2991 & & \\
\hline PBFFS & 280 & 1.2444 & & & PBFFS & 267 & 1.2477 & & \\
\hline $\begin{array}{l}\text { Data set } \\
3\end{array}$ & PBFFS_SA & $\begin{array}{l}\text { Ratio } \\
\text { PBFFS_SA } \\
\text { to LB }\end{array}$ & $\begin{array}{c}\text { Ratio Best } \\
\text { SH to } \\
\text { PBFFS_SA }\end{array}$ & $\begin{array}{l}\text { Ratio } \\
\text { PBFFS to } \\
\text { PBFFS_SA }\end{array}$ & $\begin{array}{l}\text { Data set } \\
4\end{array}$ & PBFFS_SA & $\begin{array}{l}\text { Ratio } \\
\text { PBFFS_SA } \\
\text { to LB }\end{array}$ & $\begin{array}{c}\text { Ratio Best } \\
\text { SH to } \\
\text { PBFFS_SA }\end{array}$ & $\begin{array}{c}\text { Ratio } \\
\text { PBFFS to } \\
\text { PBFFS_SA }\end{array}$ \\
\hline Exp 1 & 261 & 1.1600 & 1.1264 & 1.0728 & Exp 1 & 258 & 1.2056 & 1.0736 & 1.0349 \\
\hline Exp 2 & 260 & 1.1556 & 1.1308 & 1.0769 & Exp 2 & 245 & 1.1449 & 1.1306 & 1.0898 \\
\hline Exp 3 & 266 & 1.1822 & 1.1053 & 1.0526 & Exp 3 & 255 & 1.1916 & 1.0863 & 1.0471 \\
\hline Exp 4 & 264 & 1.1733 & 1.1136 & 1.0606 & Exp 4 & 254 & 1.1869 & 1.0906 & 1.0512 \\
\hline Exp 5 & 263 & 1.1689 & 1.1179 & 1.0646 & Exp 5 & 256 & 1.1963 & 1.0820 & 1.0430 \\
\hline Exp 6 & 263 & 1.1689 & 1.1179 & 1.0646 & Exp 6 & 256 & 1.1963 & 1.0820 & 1.0430 \\
\hline Exp 7 & 268 & 1.1911 & 1.0970 & 1.0448 & Exp 7 & 254 & 1.1869 & 1.0906 & 1.0512 \\
\hline Exp 8 & 266 & 1.1822 & 1.1053 & 1.0526 & Exp 8 & 256 & 1.1963 & 1.0820 & 1.0430 \\
\hline Exp 9 & 266 & 1.1822 & 1.1053 & 1.0526 & Exp 9 & 257 & 1.2009 & 1.0778 & 1.0389 \\
\hline Exp 10 & 264 & 1.1733 & 1.1136 & 1.0606 & Exp 10 & 255 & 1.1916 & 1.0863 & 1.0471 \\
\hline
\end{tabular}




\begin{tabular}{|c|c|c|c|c|c|c|c|c|c|}
\hline \multicolumn{2}{|l|}{ Data set 5} & \multirow[t]{2}{*}{$\begin{array}{c}\text { Ratio to } \\
\text { LB }\end{array}$} & \multirow{2}{*}{\multicolumn{2}{|c|}{$\begin{array}{c}\text { Stage Configuration } \\
2-1-2-1\end{array}$}} & \multicolumn{2}{|c|}{ Data set 6} & \multirow[t]{2}{*}{$\begin{array}{c}\text { Ratio to } \\
\text { LB }\end{array}$} & \multirow{2}{*}{\multicolumn{2}{|c|}{$\begin{array}{c}\text { Stage Configuration } \\
2-1-2-1\end{array}$}} \\
\hline LB & 248 & & & & LB & 234 & & & \\
\hline $\mathrm{SH} 1$ & 312 & 1.2581 & \multicolumn{2}{|c|}{ Buffers size $=3$} & $\mathrm{SH} 1$ & 307 & 1.3120 & \multicolumn{2}{|c|}{ Buffers size $=3$} \\
\hline $\mathrm{SH} 2$ & 319 & 1.2863 & & & $\mathrm{SH} 2$ & 294 & 1.2564 & & \\
\hline PBFFS & 302 & 1.2177 & & & PBFFS & 282 & 1.2051 & & \\
\hline $\begin{array}{l}\text { Data set } \\
5\end{array}$ & PBFFS_SA & $\begin{array}{c}\text { Ratio } \\
\text { PBFFS_SA } \\
\text { to LB }\end{array}$ & $\begin{array}{c}\text { Ratio Best } \\
\text { SH to } \\
\text { PBFFS_SA }\end{array}$ & $\begin{array}{c}\text { Ratio } \\
\text { PBFFS to } \\
\text { PBFFS_SA }\end{array}$ & $\begin{array}{l}\text { Data } \\
\text { set } 6\end{array}$ & PBFFS_SA & $\begin{array}{l}\text { Ratio } \\
\text { PBFFS_SA } \\
\text { to LB }\end{array}$ & $\begin{array}{l}\text { Ratio Best } \\
\text { SH to } \\
\text { PBFFS_SA }\end{array}$ & $\begin{array}{l}\text { Ratio } \\
\text { PBFFS to } \\
\text { PBFFS_SA }\end{array}$ \\
\hline Exp 1 & 297 & 1.1976 & $1.05 \overline{05}$ & 1.0168 & Exp 1 & 270 & 1.1538 & 1.0889 & $1.04 \overline{44}$ \\
\hline Exp 2 & 300 & 1.2097 & 1.0400 & 1.0067 & Exp 2 & 271 & 1.1581 & 1.0849 & 1.0406 \\
\hline Exp 3 & 293 & 1.1815 & 1.0648 & 1.0307 & Exp 3 & 277 & 1.1838 & 1.0614 & 1.0181 \\
\hline Exp 4 & 294 & 1.1855 & 1.0612 & 1.0272 & Exp 4 & 277 & 1.1838 & 1.0614 & 1.0181 \\
\hline Exp 5 & 290 & 1.1694 & 1.0759 & 1.0414 & Exp 5 & 277 & 1.1838 & 1.0614 & 1.0181 \\
\hline Exp 6 & 293 & 1.1815 & 1.0648 & 1.0307 & Exp 6 & 275 & 1.1752 & 1.0691 & 1.0255 \\
\hline $\operatorname{Exp} 7$ & 301 & 1.2137 & 1.0365 & 1.0033 & Exp 7 & 277 & 1.1838 & 1.0614 & 1.0181 \\
\hline Exp 8 & 291 & 1.1734 & 1.0722 & 1.0378 & Exp 8 & 275 & 1.1752 & 1.0691 & 1.0255 \\
\hline Exp 9 & 291 & 1.1734 & 1.0722 & 1.0378 & Exp 9 & 277 & 1.1838 & 1.0614 & 1.0181 \\
\hline Exp 10 & 292 & 1.1774 & 1.0685 & 1.0342 & Exp 10 & 277 & 1.1838 & 1.0614 & 1.0181 \\
\hline
\end{tabular}




\begin{tabular}{|c|c|c|c|c|c|c|c|c|c|}
\hline \multicolumn{2}{|c|}{ Data set 7} & $\begin{array}{c}\text { Ratio to } \\
\text { LB }\end{array}$ & \multirow{2}{*}{\multicolumn{2}{|c|}{$\begin{array}{c}\text { Stage Configuration } \\
2-1-2-1 \\
\end{array}$}} & \multicolumn{2}{|c|}{ Data set 8} & \multirow[t]{2}{*}{ Ratio to LB } & \multirow{2}{*}{\multicolumn{2}{|c|}{$\begin{array}{c}\text { Stage Configuration } \\
2-1-2-1 \\
\end{array}$}} \\
\hline LB & 224 & & & & LB & 239 & & & \\
\hline $\mathrm{SH} 1$ & 297 & 1.3259 & \multicolumn{2}{|c|}{ Buffers size $=3$} & $\mathrm{SH} 1$ & 304 & 1.2720 & \multicolumn{2}{|c|}{ Buffers size $=3$} \\
\hline $\mathrm{SH} 2$ & 288 & 1.2857 & & & $\mathrm{SH} 2$ & 307 & 1.2845 & & \\
\hline PBFFS & 276 & 1.2321 & & & PBFFS & 294 & 1.2301 & & \\
\hline $\begin{array}{l}\text { Data } \\
\text { set } 7\end{array}$ & PBFFS_SA & $\begin{array}{c}\text { Ratio } \\
\text { PBFFS_SA } \\
\text { to LB }\end{array}$ & $\begin{array}{c}\text { Ratio Best } \\
\text { SH to } \\
\text { PBFFS_SA }\end{array}$ & $\begin{array}{l}\text { Ratio } \\
\text { PBFFS to } \\
\text { PBFFS_SA }\end{array}$ & $\begin{array}{l}\text { Data } \\
\text { set } 8\end{array}$ & PBFFS_SA & $\begin{array}{c}\text { Ratio } \\
\text { PBFFS_SA } \\
\text { to LB }\end{array}$ & $\begin{array}{c}\text { Ratio Best } \\
\text { SH to } \\
\text { PBFFS_SA }\end{array}$ & $\begin{array}{c}\text { Ratio PBFFS } \\
\text { to } \\
\text { PBFFS_SA }\end{array}$ \\
\hline Exp 1 & 262 & 1.1696 & 1.0992 & 1.0534 & Exp 1 & 283 & 1.1841 & 1.0742 & 1.0389 \\
\hline Exp 2 & 262 & 1.1696 & 1.0992 & 1.0534 & Exp 2 & 279 & 1.1674 & 1.0896 & 1.0538 \\
\hline Exp 3 & 270 & 1.2054 & 1.0667 & 1.0222 & Exp 3 & 278 & 1.1632 & 1.0935 & 1.0576 \\
\hline Exp 4 & 269 & 1.2009 & 1.0706 & 1.0260 & Exp 4 & 281 & 1.1757 & 1.0819 & 1.0463 \\
\hline Exp 5 & 263 & 1.1741 & 1.0951 & 1.0494 & Exp 5 & 289 & 1.2092 & 1.0519 & 1.0173 \\
\hline Exp 6 & 265 & 1.1830 & 1.0868 & 1.0415 & Exp 6 & 283 & 1.1841 & 1.0742 & 1.0389 \\
\hline Exp 7 & 268 & 1.1964 & 1.0746 & 1.0299 & Exp 7 & 283 & 1.1841 & 1.0742 & 1.0389 \\
\hline Exp 8 & 259 & 1.1563 & 1.1120 & 1.0656 & Exp 8 & 284 & 1.1883 & 1.0704 & 1.0352 \\
\hline Exp 9 & 264 & 1.1786 & 1.0909 & 1.0455 & Exp 9 & 279 & 1.1674 & 1.0896 & 1.0538 \\
\hline Exp 10 & 266 & 1.1875 & 1.0827 & 1.0376 & Exp 10 & 289 & 1.2092 & 1.0519 & 1.0173 \\
\hline
\end{tabular}




\begin{tabular}{|c|c|c|c|c|c|c|c|c|c|}
\hline \multicolumn{2}{|c|}{ Data set 9} & \multirow[t]{2}{*}{ Ratio to LB } & \multirow{2}{*}{\multicolumn{2}{|c|}{$\begin{array}{c}\text { Stage Configuration } \\
2-1-2-1\end{array}$}} & \multicolumn{2}{|c|}{ Data set 10} & \multirow[t]{2}{*}{$\begin{array}{c}\text { Ratio to } \\
\text { LB }\end{array}$} & \multirow{2}{*}{\multicolumn{2}{|c|}{$\begin{array}{c}\text { Stage Configuration } \\
2-1-2-1\end{array}$}} \\
\hline LB & 262 & & & & LB & 216 & & & \\
\hline $\mathrm{SH} 1$ & 327 & 1.2481 & \multicolumn{2}{|c|}{ Buffers size $=3$} & $\mathrm{SH} 1$ & 276 & 1.2778 & \multicolumn{2}{|c|}{ Buffers size $=3$} \\
\hline $\mathrm{SH} 2$ & 322 & 1.2290 & & & $\mathrm{SH} 2$ & 285 & 1.3194 & & \\
\hline PBFFS & 306 & 1.1679 & & & PBFFS & 267 & 1.2361 & & \\
\hline $\begin{array}{l}\text { Data } \\
\text { set } 9\end{array}$ & PBFFS_SA & $\begin{array}{l}\text { Ratio } \\
\text { PBFFS_SA } \\
\text { to LB }\end{array}$ & $\begin{array}{l}\text { Ratio Best } \\
\text { SH to } \\
\text { PBFFS_SA }\end{array}$ & $\begin{array}{l}\text { Ratio } \\
\text { PBFFS to } \\
\text { PBFFS_SA }\end{array}$ & $\begin{array}{l}\text { Data } \\
\text { set } 10\end{array}$ & PBFFS_SA & $\begin{array}{l}\text { Ratio } \\
\text { PBFFS_SA } \\
\text { to LB }\end{array}$ & $\begin{array}{l}\text { Ratio Best } \\
\text { SH to } \\
\text { PBFFS_SA }\end{array}$ & $\begin{array}{c}\text { Ratio } \\
\text { PBFFS to } \\
\text { PBFFS_SA }\end{array}$ \\
\hline Exp 1 & 298 & 1.1374 & 1.0805 & 1.0268 & Exp 1 & 254 & 1.1759 & $1.086 \overline{6}$ & $1.05 \overline{12}$ \\
\hline Exp 2 & 296 & 1.1298 & 1.0878 & 1.0338 & Exp 2 & 252 & 1.1667 & 1.0952 & 1.0595 \\
\hline Exp 3 & 298 & 1.1374 & 1.0805 & 1.0268 & Exp 3 & 251 & 1.1620 & 1.0996 & 1.0637 \\
\hline Exp 4 & 300 & 1.1450 & 1.0733 & 1.0200 & Exp 4 & 251 & 1.1620 & 1.0996 & 1.0637 \\
\hline Exp 5 & 296 & 1.1298 & 1.0878 & 1.0338 & Exp 5 & 247 & 1.1435 & 1.1174 & 1.0810 \\
\hline Exp 6 & 298 & 1.1374 & 1.0805 & 1.0268 & Exp 6 & 251 & 1.1620 & 1.0996 & 1.0637 \\
\hline $\operatorname{Exp} 7$ & 300 & 1.1450 & 1.0733 & 1.0200 & Exp 7 & 252 & 1.1667 & 1.0952 & 1.0595 \\
\hline Exp 8 & 298 & 1.1374 & 1.0805 & 1.0268 & Exp 8 & 251 & 1.1620 & 1.0996 & 1.0637 \\
\hline Exp 9 & 295 & 1.1260 & 1.0915 & 1.0373 & Exp 9 & 251 & 1.1620 & 1.0996 & 1.0637 \\
\hline Exp 10 & 301 & 1.1489 & 1.0698 & 1.0166 & Exp 10 & 248 & 1.1481 & 1.1129 & 1.0766 \\
\hline
\end{tabular}




\begin{tabular}{|c|c|c|c|c|c|c|c|c|c|}
\hline \multicolumn{2}{|c|}{ Data set 1} & Ratio to LB & \multirow{2}{*}{\multicolumn{2}{|c|}{$\begin{array}{c}\text { Stage Configuration } \\
3-3-2-2-1 \\
\end{array}$}} & \multicolumn{2}{|c|}{ Data set 2} & Ratio to LB & \multirow{2}{*}{\multicolumn{2}{|c|}{$\begin{array}{c}\text { Stage Configuration } \\
3-3-2-2-1 \\
\end{array}$}} \\
\hline LB & 214 & - & & & LB & 243 & - & & \\
\hline $\mathrm{SH} 1$ & 313 & 1.4626 & \multicolumn{2}{|c|}{ Buffers size $=4$} & $\mathrm{SH} 1$ & 334 & 1.3745 & \multicolumn{2}{|c|}{ Buffers size $=4$} \\
\hline $\mathrm{SH} 2$ & 315 & 1.4720 & & & $\mathrm{SH} 2$ & 348 & 1.4321 & & \\
\hline RBFFS & 320 & 1.4953 & & & RBFFS & 351 & 1.4444 & & \\
\hline $\begin{array}{l}\text { Data set } \\
1\end{array}$ & RBFFS_SA & $\begin{array}{c}\text { Ratio } \\
\text { RBFFS_SA } \\
\text { to LB }\end{array}$ & $\begin{array}{l}\text { Ratio Best } \\
\text { SH to } \\
\text { RBFFS_SA }\end{array}$ & $\begin{array}{c}\text { Ratio } \\
\text { RBFFS to } \\
\text { RBFFS_SA }\end{array}$ & $\begin{array}{l}\text { Data set } \\
2\end{array}$ & RBFFS_SA & $\begin{array}{l}\text { Ratio } \\
\text { RBFFS_SA } \\
\text { to LB }\end{array}$ & $\begin{array}{l}\text { Ratio Best } \\
\text { SH to } \\
\text { RBFFS_SA }\end{array}$ & $\begin{array}{c}\text { Ratio } \\
\text { RBFFS to } \\
\text { RBFFS_SA }\end{array}$ \\
\hline Exp 1 & 292 & 1.3645 & 1.0719 & 1.0959 & Exp 1 & 328 & 1.3498 & 1.0183 & 1.0701 \\
\hline Exp 2 & 297 & 1.3879 & 1.0539 & 1.0774 & Exp 2 & 328 & 1.3498 & 1.0183 & 1.0701 \\
\hline Exp 3 & 298 & 1.3925 & 1.0503 & 1.0738 & Exp 3 & 332 & 1.3663 & 1.0060 & 1.0572 \\
\hline Exp 4 & 300 & 1.4019 & 1.0433 & 1.0667 & Exp 4 & 328 & 1.3498 & 1.0183 & 1.0701 \\
\hline Exp 5 & 295 & 1.3785 & 1.0610 & 1.0847 & Exp 5 & 324 & 1.3333 & 1.0309 & 1.0833 \\
\hline Exp 6 & 295 & 1.3785 & 1.0610 & 1.0847 & Exp 6 & 334 & 1.3745 & 1.0000 & 1.0509 \\
\hline Exp 7 & 292 & 1.3645 & 1.0719 & 1.0959 & Exp 7 & 330 & 1.3580 & 1.0121 & 1.0636 \\
\hline Exp 9 & 290 & 1.3551 & 1.0793 & 1.1034 & Exp 9 & 329 & 1.3539 & 1.0152 & 1.0669 \\
\hline Exp 10 & 292 & 1.3645 & 1.0719 & 1.0959 & Exp 10 & 326 & 1.3416 & 1.0245 & 1.0767 \\
\hline
\end{tabular}




\begin{tabular}{|c|c|c|c|c|c|c|c|c|c|}
\hline \multicolumn{2}{|c|}{ Data set 3} & Ratio to LB & \multirow{2}{*}{\multicolumn{2}{|c|}{$\begin{array}{c}\text { Stage Configuration } \\
3-3-2-2-1 \\
\end{array}$}} & \multicolumn{2}{|c|}{ Data set 4} & Ratio to LB & \multirow{2}{*}{\multicolumn{2}{|c|}{$\begin{array}{c}\text { Stage Configuration } \\
3-3-2-2-1 \\
\end{array}$}} \\
\hline LB & 226 & - & & & LB & 222 & - & & \\
\hline $\mathrm{SH} 1$ & 331 & 1.4646 & \multicolumn{2}{|c|}{ Buffers size $=4$} & $\mathrm{SH} 1$ & 305 & 1.3739 & \multicolumn{2}{|c|}{ Buffers size $=4$} \\
\hline $\mathrm{SH} 2$ & 333 & 1.4735 & & & $\mathrm{SH} 2$ & 319 & 1.4369 & & \\
\hline RBFFS & 338 & 1.4956 & & & RBFFS & 329 & 1.4820 & & \\
\hline $\begin{array}{l}\text { Data set } \\
3\end{array}$ & RBFFS_SA & $\begin{array}{c}\text { Ratio } \\
\text { RBFFS_SA } \\
\text { to LB }\end{array}$ & $\begin{array}{l}\text { Ratio Best } \\
\text { SH to } \\
\text { RBFFS_SA }\end{array}$ & $\begin{array}{c}\text { Ratio } \\
\text { RBFFS to } \\
\text { RBFFS_SA }\end{array}$ & $\begin{array}{l}\text { Data set } \\
4\end{array}$ & RBFFS_SA & $\begin{array}{l}\text { Ratio } \\
\text { RBFFS_SA } \\
\text { to LB }\end{array}$ & $\begin{array}{c}\text { Ratio Best } \\
\text { SH to } \\
\text { RBFFS_SA }\end{array}$ & $\begin{array}{c}\text { Ratio } \\
\text { RBFFS to } \\
\text { RBFFS_SA }\end{array}$ \\
\hline Exp 1 & 301 & 1.3319 & 1.0997 & 1.1229 & Exp 1 & 303 & 1.3649 & 1.0066 & 1.0858 \\
\hline Exp 2 & 311 & 1.3761 & 1.0643 & 1.0868 & Exp 2 & 306 & 1.3784 & 0.9967 & 1.0752 \\
\hline Exp 3 & 313 & 1.3850 & 1.0575 & 1.0799 & Exp 3 & 300 & 1.3514 & 1.0167 & 1.0967 \\
\hline Exp 4 & 307 & 1.3584 & 1.0782 & 1.1010 & Exp 4 & 304 & 1.3694 & 1.0033 & 1.0822 \\
\hline Exp 5 & 309 & 1.3673 & 1.0712 & 1.0939 & Exp 5 & 294 & 1.3243 & 1.0374 & 1.1190 \\
\hline Exp 6 & 306 & 1.3540 & 1.0817 & 1.1046 & Exp 6 & 303 & 1.3649 & 1.0066 & 1.0858 \\
\hline Exp 7 & 297 & 1.3142 & 1.1145 & 1.1380 & Exp 7 & 301 & 1.3559 & 1.0133 & 1.0930 \\
\hline Exp 8 & 305 & 1.3496 & 1.0852 & 1.1082 & Exp 8 & 306 & 1.3784 & 0.9967 & 1.0752 \\
\hline Exp 9 & 305 & 1.3496 & 1.0852 & 1.1082 & Exp 9 & 312 & 1.4054 & 0.9776 & 1.0545 \\
\hline Exp 10 & 309 & 1.3673 & 1.0712 & 1.0939 & Exp 10 & 304 & 1.3694 & 1.0033 & 1.0822 \\
\hline
\end{tabular}




\begin{tabular}{|c|c|c|c|c|c|c|c|c|c|}
\hline \multicolumn{2}{|c|}{ Data set 5} & Ratio to LB & \multirow{2}{*}{\multicolumn{2}{|c|}{$\begin{array}{c}\text { Stage Configuration } \\
3-3-2-2-1 \\
\end{array}$}} & \multicolumn{2}{|l|}{ Data set 6} & Ratio to LB & \multirow{2}{*}{\multicolumn{2}{|c|}{$\begin{array}{c}\text { Stage Configuration } \\
3-3-2-2-1 \\
\end{array}$}} \\
\hline LB & 264 & - & & & LB & 247 & & & \\
\hline $\mathrm{SH} 1$ & 363 & 1.3750 & \multicolumn{2}{|c|}{ Buffers size $=4$} & $\mathrm{SH} 1$ & 348 & 1.4089 & \multicolumn{2}{|c|}{ Buffers size $=4$} \\
\hline $\mathrm{SH} 2$ & 377 & 1.4280 & & & $\mathrm{SH} 2$ & 353 & 1.4291 & & \\
\hline RBFFS & 376 & 1.4242 & & & RBFFS & 361 & 1.4615 & & \\
\hline $\begin{array}{l}\text { Data set } \\
5\end{array}$ & RBFFS_SA & $\begin{array}{l}\text { Ratio } \\
\text { RBFFS_SA } \\
\text { to LB }\end{array}$ & $\begin{array}{l}\text { Ratio Best } \\
\text { SH to } \\
\text { RBFFS_SA }\end{array}$ & $\begin{array}{c}\text { Ratio } \\
\text { RBFFS to } \\
\text { RBFFS_SA }\end{array}$ & Data set 6 & RBFFS_SA & $\begin{array}{c}\text { Ratio } \\
\text { RBFFS_SA } \\
\text { to LB }\end{array}$ & $\begin{array}{l}\text { Ratio Best } \\
\text { SH to } \\
\text { RBFFS_SA }\end{array}$ & $\begin{array}{c}\text { Ratio } \\
\text { RBFFS to } \\
\text { RBFFS_SA }\end{array}$ \\
\hline Exp 1 & 355 & 1.3447 & 1.0225 & 1.0592 & Exp 1 & 339 & 1.3725 & 1.0265 & 1.0649 \\
\hline Exp 2 & 356 & 1.3485 & 1.0197 & 1.0562 & Exp 2 & 339 & 1.3725 & 1.0265 & 1.0649 \\
\hline Exp 3 & 353 & 1.3371 & 1.0283 & 1.0652 & Exp 3 & 334 & 1.3522 & 1.0419 & 1.0808 \\
\hline Exp 4 & 357 & 1.3523 & 1.0168 & 1.0532 & Exp 4 & 335 & 1.3563 & 1.0388 & 1.0776 \\
\hline Exp 5 & 348 & 1.3182 & 1.0431 & 1.0805 & Exp 5 & 333 & 1.3482 & 1.0450 & 1.0841 \\
\hline Exp 6 & 357 & 1.3523 & 1.0168 & 1.0532 & Exp 6 & 331 & 1.3401 & 1.0514 & 1.0906 \\
\hline Exp 7 & 352 & 1.3333 & 1.0313 & 1.0682 & Exp 7 & 331 & 1.3401 & 1.0514 & 1.0906 \\
\hline Exp 8 & 352 & 1.3333 & 1.0313 & 1.0682 & Exp 8 & 331 & 1.3401 & 1.0514 & 1.0906 \\
\hline Exp 9 & 346 & 1.3106 & 1.0491 & 1.0867 & Exp 9 & 332 & 1.3441 & 1.0482 & 1.0873 \\
\hline Exp 10 & 352 & 1.3333 & 1.0313 & 1.0682 & Exp 10 & 328 & 1.3279 & 1.0610 & 1.1006 \\
\hline
\end{tabular}




\begin{tabular}{|c|c|c|c|c|c|c|c|c|c|}
\hline \multicolumn{2}{|c|}{ Data set 7} & \multirow[t]{2}{*}{ Ratio to LB } & \multirow{2}{*}{\multicolumn{2}{|c|}{$\begin{array}{c}\text { Stage Configuration } \\
3-3-2-2-1 \\
\end{array}$}} & \multicolumn{2}{|c|}{ Data set 8} & \multirow[t]{2}{*}{ Ratio to LB } & \multirow{2}{*}{\multicolumn{2}{|c|}{$\begin{array}{c}\text { Stage Configuration } \\
3-3-2-2-1 \\
\end{array}$}} \\
\hline LB & 239 & & & & LB & 243 & & & \\
\hline $\mathrm{SH} 1$ & 329 & 1.3766 & \multicolumn{2}{|c|}{ Buffers size $=4$} & $\mathrm{SH} 1$ & 355 & 1.4609 & \multicolumn{2}{|c|}{ Buffers size $=4$} \\
\hline $\mathrm{SH} 2$ & 352 & 1.4728 & & & $\mathrm{SH} 2$ & 355 & 1.4609 & & \\
\hline RBFFS & 346 & 1.4477 & & & RBFFS & 365 & 1.5021 & & \\
\hline $\begin{array}{l}\text { Data set } \\
7\end{array}$ & RBFFS_SA & $\begin{array}{l}\text { Ratio } \\
\text { RBFFS_SA } \\
\text { to LB }\end{array}$ & $\begin{array}{l}\text { Ratio Best } \\
\text { SH to } \\
\text { RBFFS_SA }\end{array}$ & $\begin{array}{c}\text { Ratio } \\
\text { RBFFS to } \\
\text { RBFFS_SA }\end{array}$ & $\begin{array}{l}\text { Data set } \\
8\end{array}$ & RBFFS_SA & $\begin{array}{l}\text { Ratio } \\
\text { RBFFS_SA } \\
\text { to LB }\end{array}$ & $\begin{array}{l}\text { Ratio Best } \\
\text { SH to } \\
\text { RBFFS_SA }\end{array}$ & $\begin{array}{c}\text { Ratio } \\
\text { RBFFS to } \\
\text { RBFFS_SA }\end{array}$ \\
\hline Exp 1 & 316 & 1.3222 & 1.0411 & 1.0949 & Exp 1 & 342 & 1.4074 & 1.0380 & 1.0673 \\
\hline Exp 2 & 316 & 1.3222 & 1.0411 & 1.0949 & Exp 2 & 331 & 1.3621 & 1.0725 & 1.1027 \\
\hline Exp 3 & 317 & 1.3264 & 1.0379 & 1.0915 & Exp 3 & 336 & 1.3827 & 1.0565 & 1.0863 \\
\hline Exp 4 & 312 & 1.3054 & 1.0545 & 1.1090 & Exp 4 & 335 & 1.3786 & 1.0597 & 1.0896 \\
\hline Exp 5 & 320 & 1.3389 & 1.0281 & 1.0813 & Exp 5 & 339 & 1.3951 & 1.0472 & 1.0767 \\
\hline Exp 6 & 318 & 1.3305 & 1.0346 & 1.0881 & Exp 6 & 339 & 1.3951 & 1.0472 & 1.0767 \\
\hline Exp 7 & 317 & 1.3264 & 1.0379 & 1.0915 & Exp 7 & 330 & 1.3580 & 1.0758 & 1.1061 \\
\hline Exp 9 & 318 & 1.3305 & 1.0346 & 1.0881 & $\operatorname{Exp} 9$ & 345 & 1.4198 & 1.0290 & 1.0580 \\
\hline Exp 10 & 313 & 1.3096 & 1.0511 & 1.1054 & Exp 10 & 337 & 1.3868 & 1.0534 & 1.0831 \\
\hline
\end{tabular}




\begin{tabular}{|c|c|c|c|c|c|c|c|c|c|}
\hline \multicolumn{2}{|c|}{ Data set 9} & Ratio to LB & \multirow{2}{*}{\multicolumn{2}{|c|}{$\begin{array}{c}\text { Stage Configuration } \\
3-3-2-2-1 \\
\end{array}$}} & \multicolumn{2}{|c|}{ Data set 10} & Ratio to LB & \multirow{2}{*}{\multicolumn{2}{|c|}{$\begin{array}{c}\text { Stage Configuration } \\
3-3-2-2-1 \\
\end{array}$}} \\
\hline LB & 268 & & & & LB & 227 & & & \\
\hline $\mathrm{SH} 1$ & 378 & 1.4104 & \multicolumn{2}{|c|}{ Buffers size $=4$} & $\mathrm{SH} 1$ & 311 & 1.3700 & \multicolumn{2}{|c|}{ Buffers size $=4$} \\
\hline $\mathrm{SH} 2$ & 379 & 1.4142 & & & $\mathrm{SH} 2$ & 339 & 1.4934 & & \\
\hline RBFFS & 384 & 1.4328 & & & RBFFS & 322 & 1.4185 & & \\
\hline $\begin{array}{l}\text { Data set } \\
9\end{array}$ & RBFFS_SA & $\begin{array}{l}\text { Ratio } \\
\text { RBFFS_SA } \\
\text { to LB }\end{array}$ & $\begin{array}{l}\text { Ratio Best } \\
\text { SH to } \\
\text { RBFFS_SA }\end{array}$ & $\begin{array}{c}\text { Ratio } \\
\text { RBFFS to } \\
\text { RBFFS_SA }\end{array}$ & $\begin{array}{l}\text { Data set } \\
10\end{array}$ & RBFFS_SA & $\begin{array}{c}\text { Ratio } \\
\text { RBFFS_SA } \\
\text { to LB }\end{array}$ & $\begin{array}{c}\text { Ratio Best } \\
\text { SH to } \\
\text { RBFFS_SA }\end{array}$ & $\begin{array}{c}\text { Ratio } \\
\text { RBFFS to } \\
\text { RBFFS_SA }\end{array}$ \\
\hline Exp 1 & 364 & 1.3582 & 1.0385 & 1.0549 & Exp 1 & 302 & 1.3304 & 1.0298 & 1.0662 \\
\hline Exp 2 & 368 & 1.3731 & 1.0272 & 1.0435 & Exp 2 & 301 & 1.3260 & 1.0332 & 1.0698 \\
\hline Exp 3 & 356 & 1.3284 & 1.0618 & 1.0787 & Exp 3 & 297 & 1.3084 & 1.0471 & 1.0842 \\
\hline Exp 4 & 357 & 1.3321 & 1.0588 & 1.0756 & Exp 4 & 304 & 1.3392 & 1.0230 & 1.0592 \\
\hline Exp 5 & 364 & 1.3582 & 1.0385 & 1.0549 & Exp 5 & 303 & 1.3348 & 1.0264 & 1.0627 \\
\hline Exp 6 & 368 & 1.3731 & 1.0272 & 1.0435 & Exp 6 & 300 & 1.3216 & 1.0367 & 1.0733 \\
\hline Exp 7 & 364 & 1.3582 & 1.0385 & 1.0549 & Exp 7 & 300 & 1.3216 & 1.0367 & 1.0733 \\
\hline Exp 8 & 362 & 1.3507 & 1.0442 & 1.0608 & Exp 8 & 295 & 1.2996 & 1.0542 & 1.0915 \\
\hline Exp 9 & 361 & 1.3470 & 1.0471 & 1.0637 & Exp 9 & 295 & 1.2996 & 1.0542 & 1.0915 \\
\hline Exp 10 & 364 & 1.3582 & 1.0385 & 1.0549 & Exp 10 & 299 & 1.3172 & 1.0401 & 1.0769 \\
\hline
\end{tabular}




\begin{tabular}{|c|c|c|c|c|c|c|c|c|c|}
\hline \multicolumn{2}{|l|}{ Data set 1} & \multirow{2}{*}{$\begin{array}{c}\text { Ratio to LB } \\
-\end{array}$} & \multirow{2}{*}{\multicolumn{2}{|c|}{$\begin{array}{c}\text { Stage Configuration } \\
3-3-2-2-1\end{array}$}} & \multicolumn{2}{|c|}{ Data set 2} & \multirow{2}{*}{$\begin{array}{c}\text { Ratio to } \\
\text { LB } \\
-\end{array}$} & \multirow{2}{*}{\multicolumn{2}{|c|}{$\begin{array}{c}\text { Stage Configuration } \\
3-3-2-2-1\end{array}$}} \\
\hline LB & 214 & & & & LB & 243 & & & \\
\hline $\mathrm{SH} 1$ & 313 & 1.4626 & \multicolumn{2}{|c|}{ Buffers size $=4$} & $\mathrm{SH} 1$ & 334 & 1.3745 & \multicolumn{2}{|c|}{ Buffers size $=4$} \\
\hline $\mathrm{SH} 2$ & 315 & 1.4720 & & & $\mathrm{SH} 2$ & 348 & 1.4321 & & \\
\hline PBFFS & 297 & 1.3879 & & & PBFFS & 328 & 1.3498 & & \\
\hline Data set 1 & PBFFS_SA & $\begin{array}{l}\text { Ratio } \\
\text { PBFFS_SA } \\
\text { to LB }\end{array}$ & $\begin{array}{c}\text { Ratio Best } \\
\text { SH to } \\
\text { PBFFS_SA }\end{array}$ & $\begin{array}{l}\text { Ratio } \\
\text { PBFFS to } \\
\text { PBFFS_SA }\end{array}$ & $\begin{array}{l}\text { Data set } \\
2\end{array}$ & PBFFS_SA & $\begin{array}{l}\text { Ratio } \\
\text { PBFFS_SA } \\
\text { to LB }\end{array}$ & $\begin{array}{c}\text { Ratio Best } \\
\text { SH to } \\
\text { PBFFS_SA }\end{array}$ & $\begin{array}{c}\text { Ratio } \\
\text { PBFFS to } \\
\text { PBFFS_SA }\end{array}$ \\
\hline Exp 1 & 287 & 1.3411 & 1.0906 & 1.0348 & Exp 1 & 325 & 1.3374 & 1.0277 & 1.0092 \\
\hline Exp 2 & 287 & 1.3411 & 1.0906 & 1.0348 & Exp 2 & 319 & 1.3128 & 1.0470 & 1.0282 \\
\hline Exp 3 & 288 & 1.3458 & 1.0868 & 1.0313 & Exp 3 & 322 & 1.3251 & 1.0373 & 1.0186 \\
\hline Exp 4 & 285 & 1.3318 & 1.0982 & 1.0421 & Exp 4 & 318 & 1.3086 & 1.0503 & 1.0314 \\
\hline Exp 5 & 286 & 1.3364 & 1.0944 & 1.0385 & Exp 5 & 318 & 1.3086 & 1.0503 & 1.0314 \\
\hline Exp 6 & 286 & 1.3364 & 1.0944 & 1.0385 & Exp 6 & 319 & 1.3128 & 1.0470 & 1.0282 \\
\hline Exp 7 & 285 & 1.3318 & 1.0982 & 1.0421 & Exp 7 & 323 & 1.3292 & 1.0341 & 1.0155 \\
\hline Exp 8 & 291 & 1.3598 & 1.0756 & 1.0206 & Exp 8 & 321 & 1.3210 & 1.0405 & 1.0218 \\
\hline Exp 9 & 289 & 1.3505 & 1.0830 & 1.0277 & Exp 9 & 320 & 1.3169 & 1.0438 & 1.0250 \\
\hline Exp 10 & 289 & 1.3505 & 1.0830 & 1.0277 & Exp 10 & 321 & 1.3210 & 1.0405 & 1.0218 \\
\hline
\end{tabular}




\begin{tabular}{|c|c|c|c|c|c|c|c|c|c|}
\hline \multicolumn{2}{|c|}{ Data set 3} & $\begin{array}{c}\text { Ratio to } \\
\text { LB }\end{array}$ & \multirow{2}{*}{\multicolumn{2}{|c|}{$\begin{array}{c}\text { Stage Configuration } \\
3-3-2-2-1\end{array}$}} & Data set & & $\begin{array}{c}\text { Ratio to } \\
\text { LB }\end{array}$ & \multirow{2}{*}{\multicolumn{2}{|c|}{$\begin{array}{c}\text { Stage Configuration } \\
3-3-2-2-1\end{array}$}} \\
\hline LB & 226 & - & & & LB & 222 & - & & \\
\hline $\mathrm{SH} 1$ & 331 & 1.4646 & \multicolumn{2}{|c|}{ Buffers size $=4$} & $\mathrm{SH} 1$ & 305 & 1.3739 & \multicolumn{2}{|c|}{ Buffers size $=4$} \\
\hline $\mathrm{SH} 2$ & 333 & 1.4735 & & & $\mathrm{SH} 2$ & 319 & 1.4369 & & \\
\hline PBFFS & 326 & 1.4425 & & & PBFFS & 296 & 1.3333 & & \\
\hline $\begin{array}{l}\text { Data set } \\
3\end{array}$ & PBFFS_SA & $\begin{array}{l}\text { Ratio } \\
\text { PBFFS_SA } \\
\text { to LB }\end{array}$ & $\begin{array}{l}\text { Ratio Best } \\
\text { SH to } \\
\text { PBFFS_SA }\end{array}$ & $\begin{array}{c}\text { Ratio } \\
\text { PBFFS to } \\
\text { PBFFS_SA }\end{array}$ & $\begin{array}{l}\text { Data set } \\
4\end{array}$ & PBFFS_SA & $\begin{array}{l}\text { Ratio } \\
\text { PBFFS_SA } \\
\text { to LB }\end{array}$ & $\begin{array}{l}\text { Ratio Best } \\
\text { SH to } \\
\text { PBFFS_SA }\end{array}$ & $\begin{array}{c}\text { Ratio } \\
\text { PBFFS to } \\
\text { PBFFS_SA }\end{array}$ \\
\hline Exp 1 & 296 & 1.3097 & 1.1182 & 1.1014 & Exp 1 & 294 & 1.3243 & 1.0374 & 1.0068 \\
\hline Exp 2 & 292 & 1.2920 & 1.1336 & 1.1164 & Exp 2 & 295 & 1.3288 & 1.0339 & 1.0034 \\
\hline Exp 3 & 295 & 1.3053 & 1.1220 & 1.1051 & Exp 3 & 290 & 1.3063 & 1.0517 & 1.0207 \\
\hline Exp 4 & 295 & 1.3053 & 1.1220 & 1.1051 & Exp 4 & 292 & 1.3153 & 1.0445 & 1.0137 \\
\hline $\operatorname{Exp} 5$ & 296 & 1.3097 & 1.1182 & 1.1014 & $\operatorname{Exp} 5$ & 294 & 1.3243 & 1.0374 & 1.0068 \\
\hline Exp 6 & 293 & 1.2965 & 1.1297 & 1.1126 & Exp 6 & 293 & 1.3198 & 1.0410 & 1.0102 \\
\hline Exp 7 & 296 & 1.3097 & 1.1182 & 1.1014 & Exp 7 & 294 & 1.3243 & 1.0374 & 1.0068 \\
\hline Exp 8 & 298 & 1.3186 & 1.1107 & 1.0940 & Exp 8 & 296 & 1.3333 & 1.0304 & 1.0000 \\
\hline Exp 9 & 292 & 1.2920 & 1.1336 & 1.1164 & Exp 9 & 294 & 1.3243 & 1.0374 & 1.0068 \\
\hline Exp 10 & 293 & 1.2965 & 1.1297 & 1.1126 & Exp 10 & 290 & 1.3063 & 1.0517 & 1.0207 \\
\hline
\end{tabular}




\begin{tabular}{|c|c|c|c|c|c|c|c|c|c|}
\hline \multicolumn{2}{|c|}{ Data set 5} & $\begin{array}{c}\text { Ratio to } \\
\text { LB }\end{array}$ & \multirow{2}{*}{\multicolumn{2}{|c|}{$\begin{array}{c}\text { Stage Configuration } \\
3-3-2-2-1\end{array}$}} & \multicolumn{2}{|c|}{ Data set 6} & $\begin{array}{c}\text { Ratio to } \\
\text { LB }\end{array}$ & \multirow{2}{*}{\multicolumn{2}{|c|}{$\begin{array}{c}\text { Stage Configuration } \\
3-3-2-2-1\end{array}$}} \\
\hline LB & 264 & - & & & LB & 247 & & & \\
\hline $\mathrm{SH} 1$ & 363 & 1.3750 & \multicolumn{2}{|c|}{ Buffers size $=4$} & $\mathrm{SH} 1$ & 348 & 1.4089 & \multicolumn{2}{|c|}{ Buffers size $=4$} \\
\hline $\mathrm{SH} 2$ & 377 & 1.4280 & & & $\mathrm{SH} 2$ & 353 & 1.4291 & & \\
\hline PBFFS & 358 & 1.3561 & & & PBFFS & 330 & 1.3360 & & \\
\hline $\begin{array}{l}\text { Data set } \\
5\end{array}$ & PBFFS_SA & $\begin{array}{c}\text { Ratio } \\
\text { PBFFS_SA } \\
\text { to LB }\end{array}$ & $\begin{array}{l}\text { Ratio Best } \\
\text { SH to } \\
\text { PBFFS_SA }\end{array}$ & $\begin{array}{c}\text { Ratio } \\
\text { PBFFS to } \\
\text { PBFFS_SA }\end{array}$ & $\begin{array}{l}\text { Data } \\
\text { set } 6\end{array}$ & PBFFS_SA & $\begin{array}{c}\text { Ratio } \\
\text { PBFFS_SA } \\
\text { to LB }\end{array}$ & $\begin{array}{l}\text { Ratio Best } \\
\text { SH to } \\
\text { PBFFS_SA }\end{array}$ & $\begin{array}{c}\text { Ratio } \\
\text { PBFFS to } \\
\text { PBFFS_SA }\end{array}$ \\
\hline Exp 1 & 339 & 1.2841 & 1.0708 & 1.0560 & Exp 1 & 324 & 1.3117 & 1.0741 & 1.0185 \\
\hline Exp 2 & 345 & 1.3068 & 1.0522 & 1.0377 & Exp 2 & 318 & 1.2874 & 1.0943 & 1.0377 \\
\hline Exp 3 & 343 & 1.2992 & 1.0583 & 1.0437 & Exp 3 & 324 & 1.3117 & 1.0741 & 1.0185 \\
\hline Exp 4 & 340 & 1.2879 & 1.0676 & 1.0529 & Exp 4 & 323 & 1.3077 & 1.0774 & 1.0217 \\
\hline Exp 5 & 340 & 1.2879 & 1.0676 & 1.0529 & Exp 5 & 323 & 1.3077 & 1.0774 & 1.0217 \\
\hline Exp 6 & 342 & 1.2955 & 1.0614 & 1.0468 & Exp 6 & 324 & 1.3117 & 1.0741 & 1.0185 \\
\hline Exp 7 & 343 & 1.2992 & 1.0583 & 1.0437 & Exp 7 & 324 & 1.3117 & 1.0741 & 1.0185 \\
\hline Exp 8 & 347 & 1.3144 & 1.0461 & 1.0317 & Exp 8 & 320 & 1.2955 & 1.0875 & 1.0313 \\
\hline Exp 9 & 345 & 1.3068 & 1.0522 & 1.0377 & Exp 9 & 321 & 1.2996 & 1.0841 & 1.0280 \\
\hline Exp 10 & 336 & 1.2727 & 1.0804 & 1.0655 & Exp 10 & 324 & 1.3117 & 1.0741 & 1.0185 \\
\hline
\end{tabular}




\begin{tabular}{|c|c|c|c|c|c|c|c|c|c|}
\hline \multicolumn{2}{|c|}{ Data set 7} & \multirow[t]{2}{*}{$\begin{array}{c}\text { Ratio to } \\
\text { LB }\end{array}$} & \multirow{2}{*}{\multicolumn{2}{|c|}{$\begin{array}{c}\text { Stage Configuration } \\
3-3-2-2-1 \\
\end{array}$}} & \multicolumn{2}{|c|}{ Data set 8} & \multirow[t]{2}{*}{ Ratio to LB } & \multirow{2}{*}{\multicolumn{2}{|c|}{$\begin{array}{c}\text { Stage Configuration } \\
3-3-2-2-1 \\
\end{array}$}} \\
\hline LB & 239 & & & & LB & 243 & & & \\
\hline $\mathrm{SH} 1$ & 329 & 1.3766 & \multicolumn{2}{|c|}{ Buffers size $=4$} & $\mathrm{SH} 1$ & 355 & 1.4609 & \multicolumn{2}{|c|}{ Buffers size $=4$} \\
\hline $\mathrm{SH} 2$ & 352 & 1.4728 & & & $\mathrm{SH} 2$ & 355 & 1.4609 & & \\
\hline PBFFS & 323 & 1.3515 & & & PBFFS & 342 & 1.4074 & & \\
\hline $\begin{array}{l}\text { Data set } \\
7\end{array}$ & PBFFS_SA & $\begin{array}{l}\text { Ratio } \\
\text { PBFFS_SA } \\
\text { to LB }\end{array}$ & $\begin{array}{c}\text { Ratio Best } \\
\text { SH to } \\
\text { PBFFS_SA }\end{array}$ & $\begin{array}{c}\text { Ratio } \\
\text { PBFFS to } \\
\text { PBFFS_SA }\end{array}$ & $\begin{array}{l}\text { Data set } \\
8\end{array}$ & PBFFS_SA & $\begin{array}{l}\text { Ratio } \\
\text { PBFFS_SA } \\
\text { to LB }\end{array}$ & $\begin{array}{c}\text { Ratio Best } \\
\text { SH to } \\
\text { PBFFS_SA }\end{array}$ & $\begin{array}{c}\text { Ratio PBFFS } \\
\text { to } \\
\text { PBFFS_SA }\end{array}$ \\
\hline Exp 1 & 311 & 1.3013 & 1.0579 & 1.0386 & Exp 1 & 326 & 1.3416 & 1.0890 & 1.0491 \\
\hline Exp 2 & 308 & 1.2887 & 1.0682 & 1.0487 & Exp 2 & 331 & 1.3621 & 1.0725 & 1.0332 \\
\hline Exp 3 & 309 & 1.2929 & 1.0647 & 1.0453 & Exp 3 & 330 & 1.3580 & 1.0758 & 1.0364 \\
\hline $\operatorname{Exp} 4$ & 307 & 1.2845 & 1.0717 & 1.0521 & Exp 4 & 324 & 1.3333 & 1.0957 & 1.0556 \\
\hline Exp 5 & 308 & 1.2887 & 1.0682 & 1.0487 & Exp 5 & 325 & 1.3374 & 1.0923 & 1.0523 \\
\hline Exp 6 & 307 & 1.2845 & 1.0717 & 1.0521 & Exp 6 & 328 & 1.3498 & 1.0823 & 1.0427 \\
\hline $\operatorname{Exp} 7$ & 302 & 1.2636 & 1.0894 & 1.0695 & Exp 7 & 329 & 1.3539 & 1.0790 & 1.0395 \\
\hline Exp 8 & 313 & 1.3096 & 1.0511 & 1.0319 & Exp 8 & 329 & 1.3539 & 1.0790 & 1.0395 \\
\hline Exp 9 & 306 & 1.2803 & 1.0752 & 1.0556 & Exp 9 & 332 & 1.3663 & 1.0693 & 1.0301 \\
\hline Exp 10 & 310 & 1.2971 & 1.0613 & 1.0419 & Exp 10 & 334 & 1.3745 & 1.0629 & 1.0240 \\
\hline
\end{tabular}




\begin{tabular}{|c|c|c|c|c|c|c|c|c|c|}
\hline \multicolumn{2}{|c|}{ Data set 9} & \multirow[t]{2}{*}{ Ratio to LB } & \multirow{2}{*}{\multicolumn{2}{|c|}{$\begin{array}{c}\text { Stage Configuration } \\
3-3-2-2-1\end{array}$}} & \multicolumn{2}{|c|}{ Data set 10} & \multirow[t]{2}{*}{$\begin{array}{c}\text { Ratio to } \\
\text { LB }\end{array}$} & \multirow{2}{*}{\multicolumn{2}{|c|}{$\begin{array}{c}\text { Stage Configuration } \\
3-3-2-2-1\end{array}$}} \\
\hline LB & 268 & & & & LB & 227 & & & \\
\hline $\mathrm{SH} 1$ & 378 & 1.4104 & \multicolumn{2}{|c|}{ Buffers size $=4$} & $\mathrm{SH} 1$ & 311 & 1.3700 & \multicolumn{2}{|c|}{ Buffers size $=4$} \\
\hline $\mathrm{SH} 2$ & 379 & 1.4142 & & & $\mathrm{SH} 2$ & 339 & 1.4934 & & \\
\hline PBFFS & 356 & 1.3284 & & & PBFFS & 302 & 1.3304 & & \\
\hline $\begin{array}{l}\text { Data set } \\
9\end{array}$ & PBFFS_SA & $\begin{array}{l}\text { Ratio } \\
\text { PBFFS_SA } \\
\text { to LB }\end{array}$ & $\begin{array}{l}\text { Ratio Best } \\
\text { SH to } \\
\text { PBFFS_SA }\end{array}$ & $\begin{array}{l}\text { Ratio } \\
\text { PBFFS to } \\
\text { PBFFS_SA }\end{array}$ & $\begin{array}{l}\text { Data set } \\
10\end{array}$ & PBFFS_SA & $\begin{array}{c}\text { Ratio } \\
\text { PBFFS_SA } \\
\text { to LBB }\end{array}$ & $\begin{array}{c}\text { Ratio Best } \\
\text { SH to } \\
\text { PBFFS_SA }\end{array}$ & $\begin{array}{c}\text { Ratio } \\
\text { PBFFS to } \\
\text { PBFFS_SA }\end{array}$ \\
\hline Exp 1 & 350 & 1.3060 & 1.0800 & $1.017 \overline{1}$ & Exp 1 & 290 & 1.2775 & $1.07 \overline{24}$ & 1.0414 \\
\hline Exp 2 & 353 & 1.3172 & 1.0708 & 1.0085 & Exp 2 & 293 & 1.2907 & 1.0614 & 1.0307 \\
\hline Exp 3 & 351 & 1.3097 & 1.0769 & 1.0142 & Exp 3 & 293 & 1.2907 & 1.0614 & 1.0307 \\
\hline Exp 4 & 351 & 1.3097 & 1.0769 & 1.0142 & Exp 4 & 289 & 1.2731 & 1.0761 & 1.0450 \\
\hline Exp 5 & 350 & 1.3060 & 1.0800 & 1.0171 & Exp 5 & 290 & 1.2775 & 1.0724 & 1.0414 \\
\hline Exp 6 & 346 & 1.2910 & 1.0925 & 1.0289 & Exp 6 & 290 & 1.2775 & 1.0724 & 1.0414 \\
\hline $\operatorname{Exp} 7$ & 349 & 1.3022 & 1.0831 & 1.0201 & Exp 7 & 293 & 1.2907 & 1.0614 & 1.0307 \\
\hline Exp 8 & 345 & 1.2873 & 1.0957 & 1.0319 & Exp 8 & 288 & 1.2687 & 1.0799 & 1.0486 \\
\hline Exp 9 & 354 & 1.3209 & 1.0678 & 1.0056 & Exp 9 & 293 & 1.2907 & 1.0614 & 1.0307 \\
\hline Exp 10 & 352 & 1.3134 & 1.0739 & 1.0114 & Exp 10 & 291 & 1.2819 & 1.0687 & 1.0378 \\
\hline
\end{tabular}

